University of Louisville

ThinkIR: The University of Louisville's Institutional Repository

Electronic Theses and Dissertations

$12-2019$

\title{
Investigation of phosphoserine aminotransferase 1(PSAT1) in breast cancer progression.
}

Stephanie Metcalf

University of Louisville

Follow this and additional works at: https://ir.library.louisville.edu/etd

Part of the Biochemistry Commons, and the Molecular Biology Commons

\section{Recommended Citation}

Metcalf, Stephanie, "Investigation of phosphoserine aminotransferase 1(PSAT1) in breast cancer progression." (2019). Electronic Theses and Dissertations. Paper 3345.

https://doi.org/10.18297/etd/3345

This Doctoral Dissertation is brought to you for free and open access by ThinkIR: The University of Louisville's Institutional Repository. It has been accepted for inclusion in Electronic Theses and Dissertations by an authorized administrator of ThinkIR: The University of Louisville's Institutional Repository. This title appears here courtesy of the author, who has retained all other copyrights. For more information, please contact thinkir@louisville.edu. 


\title{
INVESTIGATION OF PHOSPHOSERINE AMINOTRANSFERASE 1(PSAT1) IN BREAST CANCER PROGRESSION
}

\author{
By \\ Stephanie Metcalf \\ B.A. Indiana University Purdue University Indianapolis 2014 \\ M.S. University of Louisville 2017 \\ A Dissertation \\ Submitted to the Faculty of the \\ School of Medicine at the University of Louisville \\ In Partial Fulfillment of the Requirements for the Degree of \\ Doctor of Philosophy in \\ Biochemistry and Molecular Genetics \\ Department of Biochemistry and Molecular Genetics \\ University of Louisville \\ Louisville, Kentucky
}

December 2019 

INVESTIGATION OF PHOSPHOSERINE AMINOTRANSFERASE 1(PSAT1) IN BREAST CANCER PROGRESSION

\author{
By \\ Stephanie Metcalf \\ B.A. Indiana University Purdue University Indianapolis 2014 \\ M.S. University of Louisville 2017 \\ A Dissertation Approved on \\ August 23, 2019 \\ by the Following Dissertation Committee:

\begin{tabular}{c}
\hline Brian Clem, Ph.D. \\
\hline Carolyn Klinge, Ph.D. \\
\hline David Samuelson, Ph.D. \\
\hline Paula Bates, Ph.D. \\
\hline Steve Ellis, Ph.D.
\end{tabular}




\section{DEDICATION}

This dissertation is dedicated to my parents

Mr. Elbert Dee Metcalf

and

Mrs. Luann Michelle Metcalf

who have supported me throughout my educational career. 


\section{ACKNOWLEDGEMENTS}

I would like to thank my mentor, Dr. Brian Clem, for his guidance and assistance at the beginning of my scientific career. I would also like to thank my committee members Drs. Klinge, Samuelson, Bates, and Ellis for their insights, expertise, and contribution to my scientific education. I would also like to thank all the former and current members of the Clem laboratory as well as the collaborators that have contributed to the work presented in this dissertation. Lastly, I would like to thank my family and friends for their support during the last couple of years and their understanding of the demands of obtaining a Ph.D. 


\title{
ABSTRACT \\ INVESTIGATION OF PHOSPHOSERINE AMINOTRANSFERASE 1(PSAT1) IN BREAST CANCER PROGRESSION
}

\author{
Stephanie Metcalf
}

August 23, 2019

This dissertation describes my research into the involvement of phosphoserine aminotransferase 1 (PSAT1) in breast cancer progression; specifically, in triple negative breast cancer (TNBC) metastasis and endocrine resistance in estrogen receptor positive breast cancer $(E R+B C)$. Breast cancer is the most common tumor diagnosis among women. While the overall 5-year survival for breast cancer is reaching $90 \%$, the 5 -year survival for metastatic disease is only $22 \%$. Metastasis and endocrine resistance combined can affect over $50 \%$ of patients. One of the proteins and pathways implicated in both metastasis and endocrine resistance in breast cancer is phosphoserine aminotransferase 1 (PSAT1) and the serine synthetic pathway (SSP).

From prior reports and preliminary studies within the lab, I hypothesized that PSAT1 may play a role in metastasis within TNBC and contribute to endocrine 
within ER+BC. The role of PSAT1 in TNBC metastasis was evaluated via examination of the effects of altered PSAT1 expression on metastatic potential in TNBC cell lines that were "serine synthesis-independent". Functional relevance of PSAT1 on sensitivity to endocrine therapy was tested in matched endocrine sensitive and endocrine resistant cell lines upon alteration of PSAT1 expression.

Through this work, I found that suppression of PSAT1 significantly inhibited the in vitro motility and invasiveness of "serine synthesis-independent" TNBC which was not recapitulated upon suppression of $\mathrm{PHGDH}$, which is the first enzyme within the SSP. I also found that suppression of PSAT1 reduced the number of micro-metastases within the lungs in an experimental metastasis model. In addition, I found that both PSAT1 and PHGDH correlated to poorer progression free survival in multiple patient cohorts, manipulation of PSAT1 or PHGDH in both sensitive and resistant $E R+B C$ cell lines altered sensitivity to 4-hydroxytamoxifen treatment.

This body of work has demonstrated that PSAT1 selectively promotes metastasis in "serine synthesis-independent" TNBC via a function unrelated to de novo serine synthesis. It also has shown that both PSAT1 and PHGDH contribute to tamoxifen resistance in $\mathrm{ER}+\mathrm{BC}$ and thereby implicating a role for the SSP in this context. Taken together, this dissertation demonstrates that PSAT1 contributes to breast cancer progression through promotion of TNBC metastasis and ER+BC endocrine resistance. 


\section{TABLE OF CONTENTS}

\section{Page}

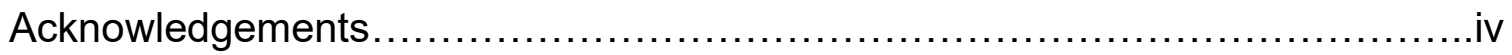

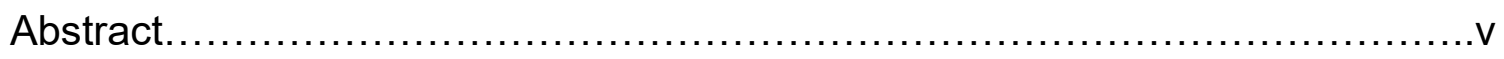

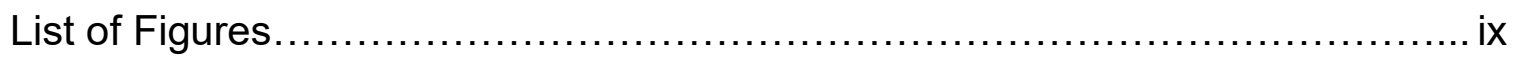

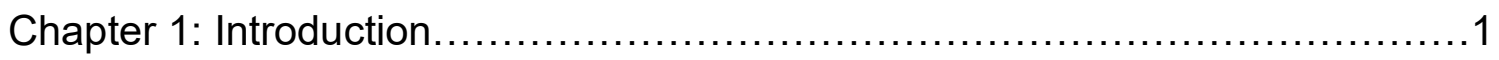

Chapter 2: Selective Loss of Phosphoserine Aminotransferase 1 (PSAT1)

Suppresses Triple Negative Breast Cancer Metastasis...........................30

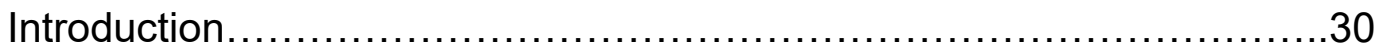

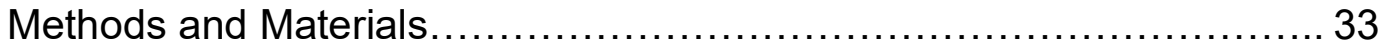

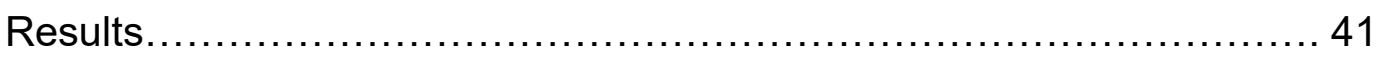

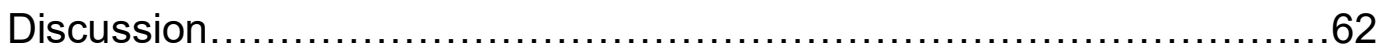

Extended Results and Discussion....................................... 67

Chapter 3: Phosphoserine Aminotransferase 1 (PSAT1) and Serine Synthesis

Pathway Alters Sensitivity to 4-Hydroxytamoxifen Treatment in Estrogen -

Receptor Positive Breast Cancer.............................................. 73

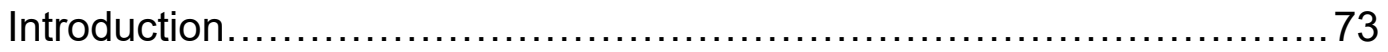

Methods and Materials................................................. 77

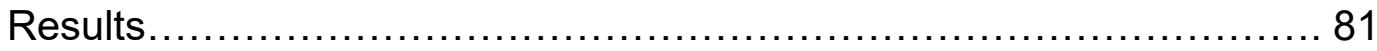

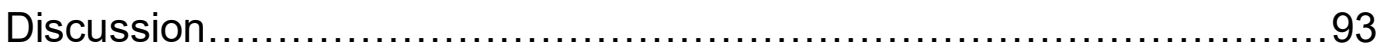

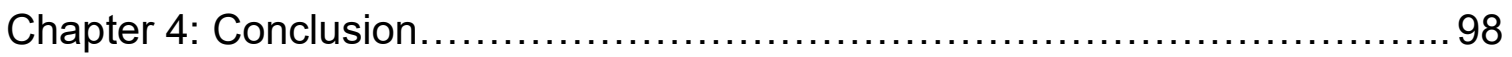




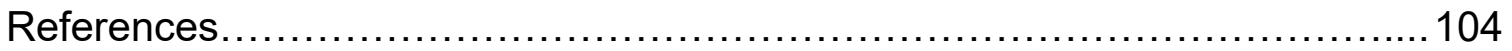

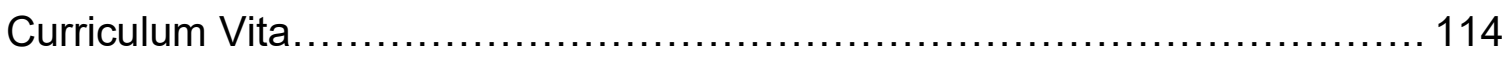




\section{LIST OF FIGURES}

$\begin{array}{lll}\text { Figure } & \text { Page }\end{array}$

1. General Subtypes of Breast Cancer.......................................... 3

2. Endocrine Therapy Mechanisms of Action................................... 5

3. The Metastatic Cascade...................................................... 7

4. Schematic of the Serine Synthesis Pathway ................................ 14

5. PSAT1 Expression Increases with TNBC Grade ............................42

6. PSAT1 and PHGDH Transcript and Protein Expression in MDA-MB-231 and HCC1806 TNBC Cell Lines................................................... 44

7. Loss of PSAT1 Does Not Affect Proliferation but alters Actin-Cytoskeleton.45

8. SiRNA suppression of PSAT1 Has No Proliferative Effect................... 46

9. Loss of PSAT1 Suppresses MDA-MB-231 Motility and Invasion In Vitro.....47

10. SiRNA suppression of PSAT1 Decreases Metastatic Potential of MDA-MB231 Cells In Vitro .......................................................... 48

11. Suppression of PSAT1 in HCC1806 Cells Does Not Have an AntiProliferative Effect............................................................. 50

12. Decreased Metastatic Potential of HCC1806 Cells Upon Suppression of PSAT1.

13. PHGDH is Dispensable for MDA-MB-231 Proliferation In Vitro................ 53 
14. SiRNA Suppression of PHGDH in MDA-MB-231 Cells Has No Effect On In

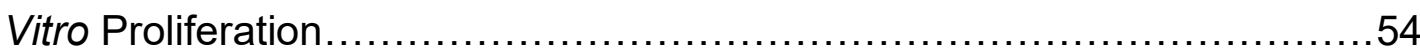

15. PHGDH Is Dispensable for MDA-MB-231 Metastasis In Vitro.................55

16. SiRNA Suppression of PHGDH in MDA-MB-231 Cells Has No Effect On

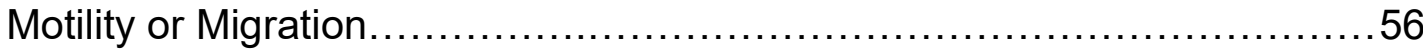

17. Loss of PHGDH Does Not Affect HCC1806 Proliferation......................57

18. Loss of PHGDH Does Not Suppress HCC1806 Motility or Migration Potential. 58

19. PSAT1 Silencing Inhibits MDA-MB-231 Experimental Metastasis............60

20. Suppression of PSAT1 Does Not Affect Anchorage-Independent Growth of MDA-MB-231 Cells ............................................................ 61

21. Exogenous Serine Does Not Rescue PSAT1 Suppression on Cell Motility 68

22. Exogenous Serine Does Not Rescue Effect of PSAT1 Suppression on Cell

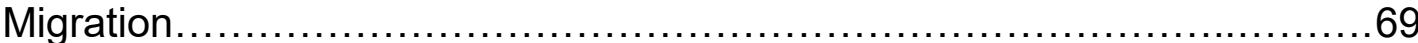

23. PSAT1 and TIMP2 are Binding Partners...................................71

24. Clinical Relevance of PSAT1 in Tamoxifen treated ER+BC $\ldots \ldots \ldots \ldots \ldots \ldots . \ldots 8$

25. Differential SSP Expression Between Sensitive and Resistant Cell Types.. 83

26. Metabolomic Differences Between Endocrine Sensitive and Endocrine

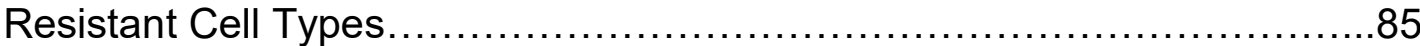

27. Overexpression of PSAT1 Reduces Sensitivity to 4-Hydroxytamoxifen......86

28. Suppression of PSAT1 Increases Sensitivity to 4-Hydroxytamoxifen.........87 87 
29. Clinical Relevance of PHGDH in ER+ Patients Treated with Tamoxifen......89

30. Suppression of PHGDH Increases to 4-Hydroxytamoxifen...................90

31. Correlation Between SSP Enzymes and Relapse-Free Survival and In Vitro Sensitivity to Endocrine Therapy is Selective for Tamoxifen..................92

32. Model of SSP Contribution to Endocrine Resistance ........................ 96 


\section{CHAPTER 1}

\section{INTRODUCTION}

Breast cancer is the most common malignancy in U.S. women, constituting $30 \%$ of all new cancer diagnoses. As the second leading cause of cancer-related deaths in women [1], it comprised $6.8 \%$ of all cancer related mortalities in 2016 [2]. The lifetime risk for a woman developing breast cancer is currently 1 in 8 or about $12 \%$ [2]. It is estimated that 268,600 women will be diagnosed with invasive breast cancer while another 62,930 will be diagnosed with non-invasive breast cancer this year [1]. At the beginning of 2019 there were in excess of 3.1 million women in the U.S. with a history of breast cancer [1]. These women though all diagnosed with breast cancer, do not all have the same disease. Breast cancer has been classified into 21 different histological subtypes that can be categorized into four main categories based on their hormone receptor $(\mathrm{HR})$ and human epidermal growth factor receptor 2 (HER2) status [3]. The four main subtypes are luminal A (HR+/HER2-), luminal B (HR+/HER2+), triple negative (HR-/HER2-), and HER2 enriched (HR-/HER2+) [3]. These all have different clinical presentations, oncogenic drivers, treatment options, affected patient populations, and confer different patient prognoses [3]. This dissertation will focus on two of these subtypes; triple negative breast cancer (TNBC) and luminal $A$, which will be referred to as estrogen receptor positive breast cancer (ER+BC). 
TNBC is characterized by lack of estrogen receptor- $\alpha(E R \alpha)$, progesterone receptor (PR), and HER2 expression [4]. This molecular subtype accounts for $10 \%$ to $20 \%$ of all breast cancer cases $[3,5]$. It is often considered to be the most aggressive with the majority of these tumors being high grade at time of diagnosis and basal-like in their appearance [6]. This aggressiveness is especially alarming considering the patient populations most commonly diagnosed with this form of cancer. TNBC is most common among women under 50 years of age, among women that are African American or Hispanic, and among women that harbor breast cancer gene 1 (BRAC1) mutations [3,7,8] (Figure 1). The aggressiveness of TNBC could be due to its heterogeneous nature and the limited treatment options, given that targeted therapies for breast cancer primarily focus on ERa and HER2. TNBCs are currently treated with a combination of surgery, chemotherapy and radiation [3]. Given the propensity of TNBC to be of a higher grade and more aggressive, there is an increased likelihood of metastasis within this subtype [5].

$\mathrm{ER}+\mathrm{BC}$ is the most common type of breast cancer observed in the clinic, accounting for approximately $60 \%$ to $75 \%$ of all breast cancer diagnoses [9]. These tumors express ER $\alpha$ and their growth and proliferation is driven by estrogen [10]. ER+ tumors are typically slow-growing and found in older patients, as most patients with ER+BC are post-menopausal [7]. This subtype also has the best prognosis, especially at early stages, due to targeted therapies that have been developed [3] (Figure 1). These therapies target different aspects of estrogen processing and can be classified into three major categories: aromatase inhibitors, selective estrogen receptor modulator (SERM), and selective estrogen receptor 


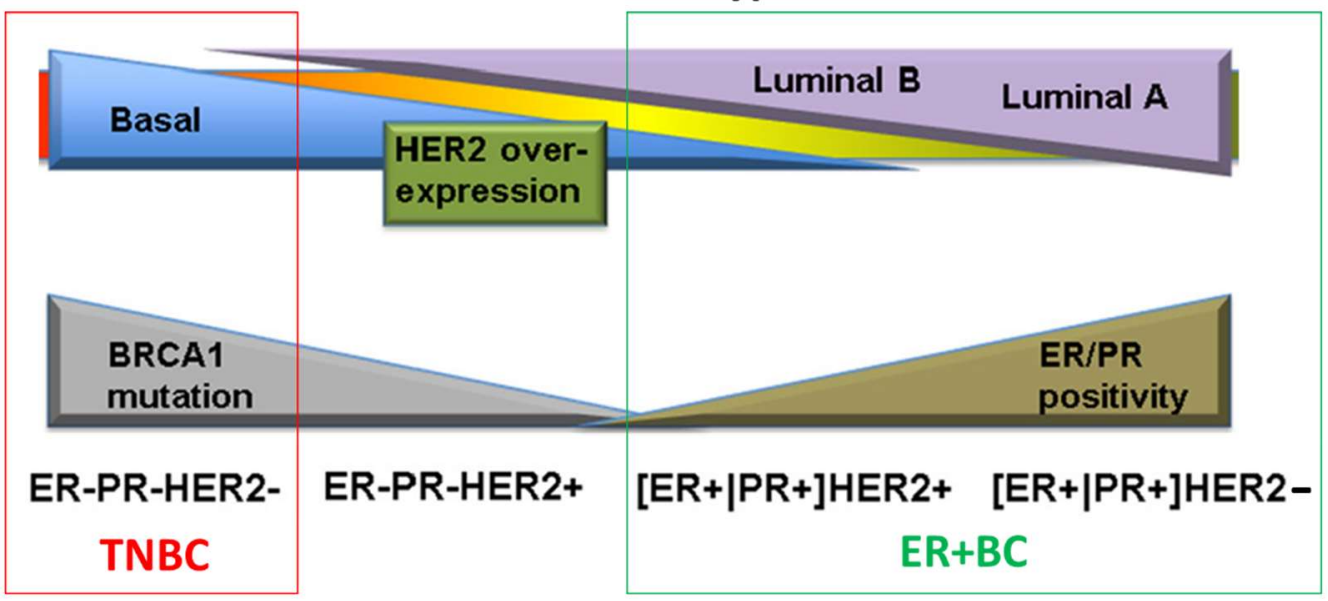

\section{Molecular Subtypes}

Figure 1: General subtypes of Breast Cancer. This schematic illustrates patient outcomes according to breast cancer subtypes. Adapted from Dai et al, 2015 [8]. Abbreviations: Estrogen Receptor (ER), Progesterone Receptor (PR), Human Epidermal Growth Factor Receptor 2 (HER2), Triple Negative Breast Cancer (TNBC), Estrogen Receptor Positive Breast Cancer (ER+BC). 
degraders (SERD), which also has anti-estrogen activity [10]. The different categories of endocrine therapy all target estrogen but do so via several different mechanisms (Figure 2). Aromatase Inhibitors, such as letrozole, prevent the conversion of androgens to estrogens [11]. Tamoxifen is a SERM and its primary mechanism of action is through competitive binding against estrogens for the estrogen receptor resulting in the inhibition of the estrogen driven pro-proliferative transcription program $[12,13]$. Fulvestrant $(\mathrm{ICl} 128,780)$ is a "pure"-antiestrogen or SERD [12]. Fulvestrant binds to the estrogen receptor and attenuates estrogen signaling through blocking estrogen binding and increased ER protein degradation [12]. Tamoxifen was the first of these targeted therapies and became the standard first-line therapy in the 1990s [9]. Since its introduction, the mortality rates associated with breast cancer have decreased significantly [3]. Much of this trend has been attributed to Tamoxifen, and its later designated replacements, that target estrogen signaling in ER+ tumors as well as improvements in detection methods [3].

With these advances in therapies and detection techniques, the current overall survival rate for female breast cancer has improved and is approaching $90 \%$; however, metastatic disease, where the overall 5 -year survival rate is $22 \%$ [2] is still an overwhelming problem. Metastases or advanced recurrent disease will develop in approximately $30 \%$ of patients despite treatment with standard and advanced therapies [14] and is responsible for $90 \%$ of breast cancer-related deaths [15]. Metastasis occurs when cells disseminate from the primary tumor site 


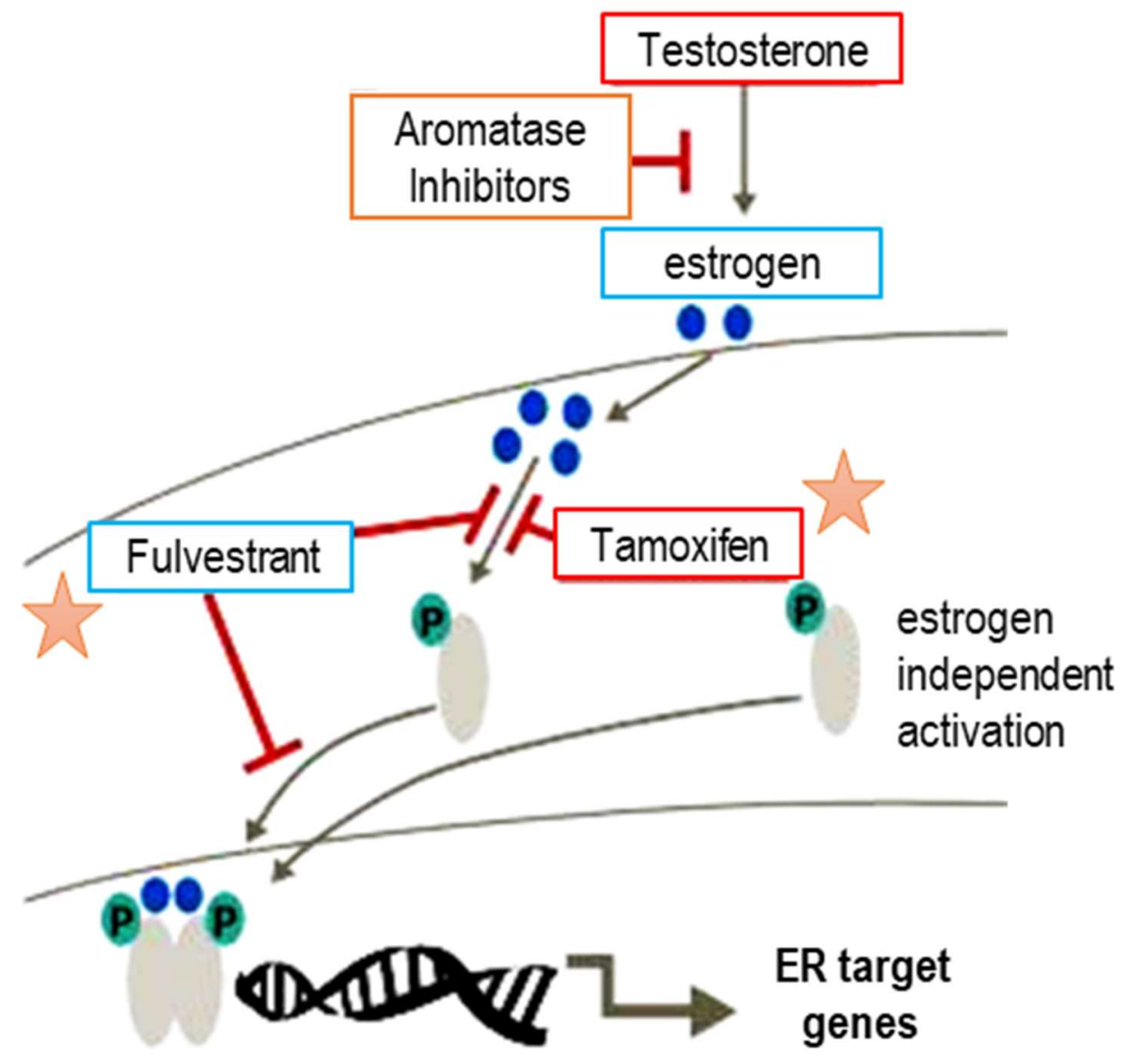

\section{Estrogen Response Elements}

Figure 2: Endocrine Therapy Mechanisms of Action. This schematic shows the mechanisms of action for common endocrine therapies. Aromatase inhibitors inhibit the conversion of testosterone to estrogen. Selective estrogen receptor modulators (SERMs), represented by Tamoxifen, compete with estrogen for binding to the estrogen receptor and block estrogen signaling. Selective estrogen receptor degraders, represented by Fulvestrant, inhibit estrogen signaling and unlike SERMs also degrade the estrogen receptor. Adapted from Schmid 2017 [9]. 
and populate a distant site. The common distant sites for breast cancer metastases are bone, liver, lung, brain, and lymph nodes, which vary depending on sub-type [16]. While metastatic breast cancer can arise from all different breast cancer subtypes [17], TNBC is considered the most aggressive as it correlates with poorer overall survival in both early stage and metastatic disease [4]. These metastases are difficult to treat and unfortunately metastatic disease is often incurable with therapies primarily directed at prolonging survival while maximizing quality of life [14]. Several clinical trials with either single agent or combinatorial therapies have only managed a 3-8 month improvement in overall survival in patients treated with the prior standard of care (anthracyclines) [14], underscoring a lack of understanding of a complex process involving numerous proteins and pathways.

The signaling pathways and processes involved in metastasis are highly regulated and together form the metastatic cascade. This is a series of steps that a cancer cell must undergo in order to spread from the primary tumor and colonize in the secondary site (Figure 3). Metastasis in its earliest form begins with the growth of a tumor within the primary organ. This requires a pro-proliferative protein signature that promotes genes utilized for growth, proliferation and the necessary angiogenesis to support this initial tumor growth [18]. Metastasis truly begins when cells disseminate from the primary tumor [19]. This dissemination requires the cells to undergo the epithelial to mesenchymal transition (EMT). EMT is a normal cellular process that is exploited by the cancer cells in order to promote motility, invasion, and other properties that are needed for the cancer cells to extend beyond the initial tumor [19]. 


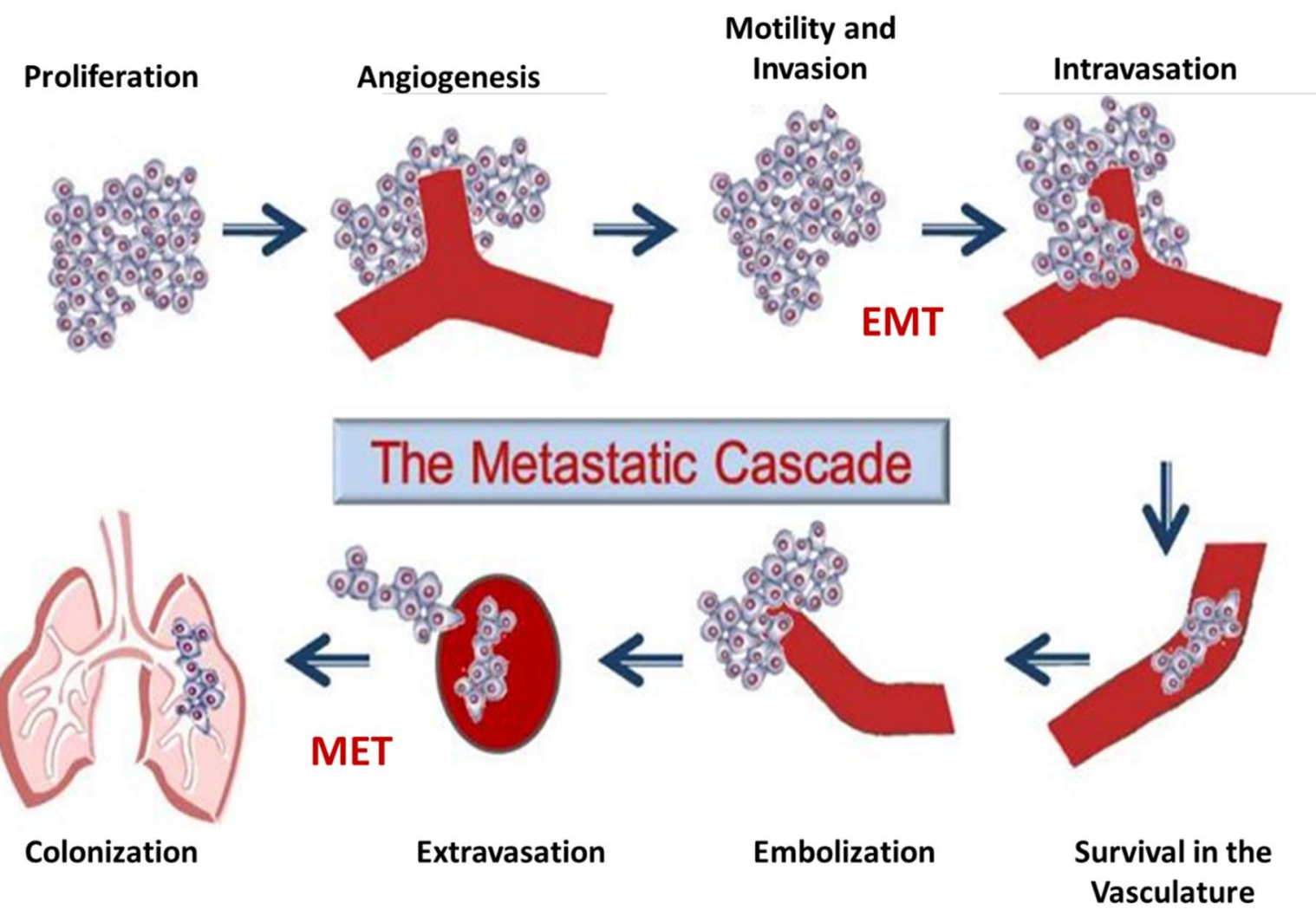

Figure 3: The Metastatic Cascade. This schematic depicts the steps involved in metastasis. First, there must be pro-proliferative signals and angiogenesis to support the growth of the primary tumor. Cells then undergo EMT which will allow for motility and invasion resulting in intravasation into the vasculature. Cells within the vasculature need to survive until they arrive at a secondary site. The cells then undergo extravasation from the vasculature, revert to their epithelial form (MET) and colonize a secondary or metastatic site. Adapted from Daves et al, 2011 [18]. 
EMT is initiated by a group of transcription factors that alter transcription of genes that aid in the invasion and motility of cells. These transcription factors include Snail, Twist, Slug, and Zeb1 [19]. During EMT, cells change dramatically. They typically experience downregulation of epithelial-like markers while simultaneously gaining mesenchymal-like markers which includes loss of cell polarity and changes in the actin cytoskeleton [20]. In order for a cell to invade into the vasculature it needs to degrade the extracellular matrix, which is the role of the matrix metalloproteinases (MMPs). The tumor cells control the expression of MMPs through several different signaling programs that provide a feed-forward mechanism for metastasis as MMPs promote the motility and invasion of cancer cells [21]. The promotion of motility and invasion is classically promoted by actin cytoskeleton regulation via Rho signaling, integrin signaling, and FAK signaling cascades [18].

Once in the vasculature, tumor cells must survive in circulation before they reach a secondary site. According to an established gene signature for metastasis, the cell is protected in these conditions by the NRF2 stress response and integrin signaling until the cell can exit circulation through the process of extravasation [18]. To complete this process, cells must invade through the endothelial wall at the secondary site, which is termed transendothelial migration (TEM) [19]. TEM is only possible when the endothelial wall has been compromised, a process requiring MMPs and VEGF [19]. After extravasation, cells must then colonize a secondary site to form a metastatic tumor. The process of colonization is extremely inefficient as experimental metastasis models have found that secondary lesions form in only 
$0.01 \%$ of instances following injection of tumor cells [19]. This is partially due to the strict requirement for the presence of growth factors at the secondary site that are necessary for tumor growth [21]. Another determination of whether a cancer cell will form a metastatic tumor is the successful completion of the mesenchymal to epithelial transition (MET). There is substantial evidence that suggest that epithelial cells have a growth advantage in a distant site compared to cells that are still mesenchymal [20]. While the general metastatic cascade is known and certain pathways have been identified as contributing to metastasis, there is still a lack of understanding as to the regulation of the process, especially in regard to unknown effectors that can be targeted to either prevent or inhibit metastasis.

Another clinical challenge for breast cancer patients is resistance to therapy. Despite advances in first line treatments, recurrent disease still develops in some breast cancer patients. This could be due to dormancy of the disseminated cells that occurs due to lack of adaption in the foreign site or lack of nutrient availability. Once the cell has adapted to the new environment it can re-enter the cell cycle and begin to proliferate again. It could also be due to tumor cells becoming resistant to therapeutic agents, such as antiestrogens for ER+ breast cancer. While endocrine resistance can ultimately lead to metastatic disease, it can also present as recurrence after treatment or as progression of the primary disease during initial therapy [11]. This phenomenon affects numerous patients, and many will go on to develop resistance to these endocrine therapies. Endocrine resistance is acquired at different rates depending on staging and time after diagnosis. Within 5 years, $10-15 \%$ of early stage ER+ patients will acquire 
resistance, at 15 years $30 \%$ of patients will have recurrent disease despite initial response to endocrine therapy. Eventually $40-50 \%$ of all ER+ patients will experience relapse, while virtually all metastatic ER+ patients will acquire resistance in 2-3 years following the start of treatment [22]. The exact mechanisms by which endocrine resistance occurs and within what patient population are not fully understood.

However, several mechanisms of endocrine resistance have been proposed. One of the mechanisms associated with resistance is dependent upon the expression of ERa. It is suggested that innate resistance is associated with patients that, while ER+, express lower levels of ERa [22]. In addition, mutations in ERa can also lead to resistance. Mutations that lead to constitutive activation of ER without the presence of a ligand would allow the cell to be resistant to both aromatase inhibitors and antiestrogens [11]. Endocrine resistance has also been linked to lack of PR expression in ER+ patients [22]. These patients have a poorer overall survival and often have innate resistance to endocrine therapies [22].

Resistance is also attributed to several other mechanisms that are not associated with the hormone receptors. Altered Tamoxifen metabolism has been linked to resistance to Tamoxifen therapy and is reliant upon the patient's CYP2DG allele [11]. Differential growth factor signaling is also implicated in endocrine resistance [11,22]. Most ER+ tumors that also express HER2 are resistant to endocrine therapy [22]. There is a substantial amount of evidence that supports crosstalk between ER, PI3K, AKT, and mTOR and that this association is linked to resistance due to aberrant activation of these pathways which reduce levels of ER 
$[11,22]$. Studies have shown that patients who responded well to endocrine therapy had altered expression of the proteome compared to non-responding patients. These changes in expression have been attributed to epigenetic regulation of proteins, differences in transcriptional programs, and changes in expression levels of the ER coregulatory proteins [11,22]. A large majority of these proteins control the cell cycle or are associated with metabolism [22].

Altered metabolism has been deemed one of the hallmarks of cancer [23]. The differential metabolism associated with cancer cells not only contributes to initial tumorigenesis but has been established as a factor in therapy resistance and metastasis [24]. The notion that cancer cells differently utilize the metabolic processes was first proposed by Dr. Otto Warburg when he noted in the early 1920s that cancer cells preferentially metabolize glucose to lactate even in the presence of oxygen for oxidative phosphorylation (Warburg effect) $[25,26]$. These changes in metabolism allow the cell to use the fuel sources available to them in nutrient-limiting conditions present in the tumor microenvironment in order to continue to proliferate and survive [24]. Tumors exploit metabolic pathways, namely glycolysis, by increasing the expression or activity of transporters and enzymes responsible for catalyzing the different metabolic reactions in order to generate building blocks (lipids, amino acid, and nucleotides) needed to sustain proliferation [24]. In glycolysis, this is achieved via an upregulation of the glucose transporters to increase glucose uptake coupled with upregulation of glycolytic enzymes or expression of alternative cancer-specific isoforms (i.e. hexokinase 2) in order to increase flux through the glycolytic pathway at a rate greater than what 
is required for pyruvate oxidation which leads to increased pyruvate generation [24]. Additionally, cancer cells also express an alternative isoform of pyruvate kinase (PKM2) which is less efficient and causes an accumulation of glycolytic intermediates. This, in turn, increases flux into the glycolytic branching pathways $[24,27,28]$.

One of those glycolytic branching pathways leads to the de novo synthesis of serine. There are several different metabolic fates of serine that aid in cell survival and proliferation including carbon support for production of cysteine, phospholipid head groups, sphingosine, glycine, glutathione, and purines [28]. Serine can also contribute to redox homeostasis via the reduction of NADPH and can feed into one-carbon metabolism to support histone, DNA, and RNA methylation as a result of SAM generation [29]. In addition, serine also serves as an allosteric activator of PKM, wherein as serine levels increase it promotes PKM2 into its tetrameric form. This form is more active and drives pyruvate generation. Conversely, when serine levels are low, PKM2 is in a dimeric form and causes the accumulation of glycolytic intermediates described above [28]. Cells can obtain serine through either de novo synthesis or uptake via serine transporters. An individual cell's pool of serine is largely determined by the tissue of origin which can determine serine consumption through the expression of the serine transporters [30]. While most cancer cells can import serine, there seems to be a preference for de novo serine synthesis, so much so that increased activation of the serine synthesis pathway (SSP) is now an established component of cancer metabolism [29,31]. In addition, the SSP is the metabolic pathway that allows for 
simultaneous utilization of glucose and glutamine as fuel sources to generate serine and intermediates that feed into the TCA cycle. The SSP is a series of three reactions that originate from the glycolytic intermediate 3-phophoglycerate and accounts for approximately $10 \%$ of 3-phosphoglycerate that is metabolized [32]. Phosphoglycerate dehydrogenase $(\mathrm{PHGDH})$ is the initiating reaction as it catalyzes the conversion of 3-phosphoglycerate to 3-phosphopyruvate [33]. The PHGDH reaction in most tissue types is the rate-limiting step and determines the overall flux through the pathway. Phosphoserine aminotransferase 1 (PSAT1) catalyzes the second step in the serine synthesis pathway through conversion of 3-phosphohydroxypyruvate to 3-phosphoserine while interconverting glutamate to a-ketoglutarate [28]. The last reaction within this pathway is the conversion of 3phosphoserine to serine which is catalyzed by the enzyme phosphoserine phosphatase (PSPH) [28] (Figure 4). This pathway is utilized differently based on tissue type in that some tissues do not express enzymes within this pathway, whereas other tissues rely heavily on de novo serine production.

In the liver, the enzymes within this pathway are expressed at relatively high levels compared to other tissue types [34] and are subject to regulation by diet and hormone changes [35]. It has been shown that the expression levels of PHGDH and PSPH can be directly regulated based on the protein and carbohydrate intake ratios in the diet [35]. Another difference in normal liver is that this pathway is not regulated by the activity of PHGDH. Flux through the SSP in the liver is determined by the activity of PSPH and is driven completely by the cellular need for serine [35]. 


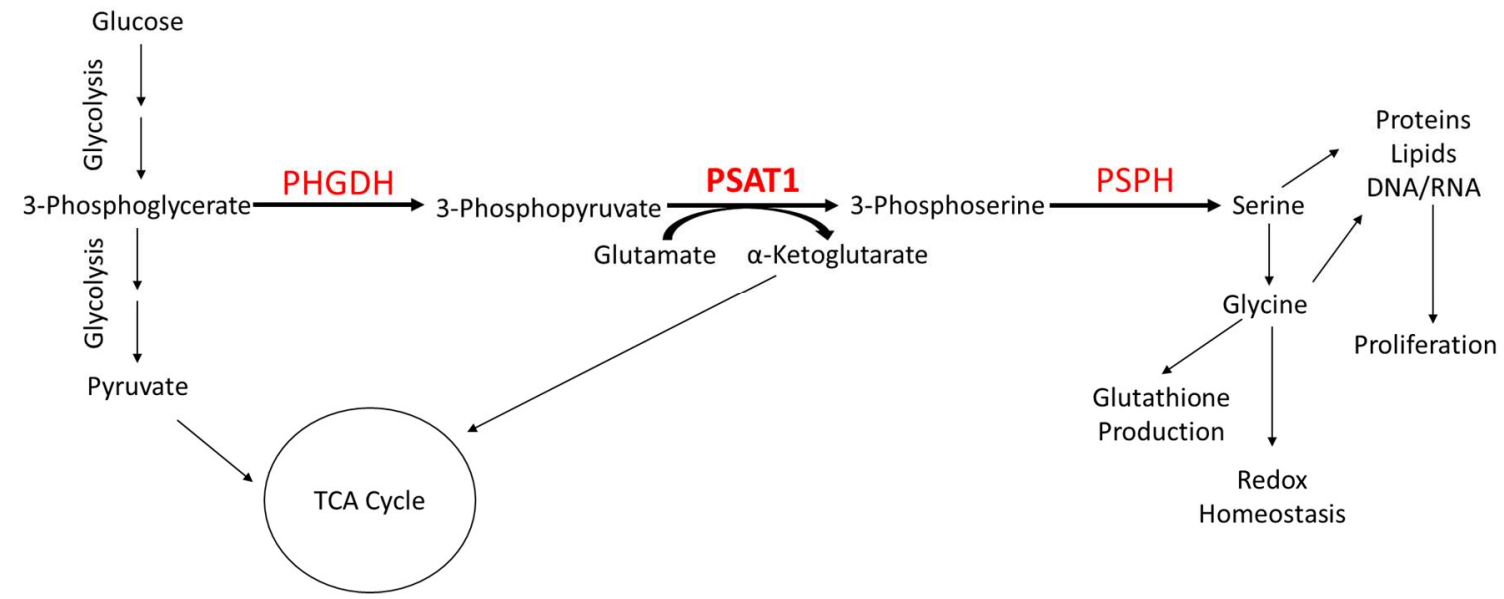

Figure 4: Schematic of the Serine Synthesis Pathway. This schematic illustrates the three-step serine synthesis pathway and its involvement in downstream processes. This pathway is catalyzed by the enzymes phosphoglycerate dehydrogenase (PHGDH), phosphoserine aminotransferase 1 (PSAT1), and phosphoserine phosphatase (PSPH). 
The SSP in the brain also differs from most other tissue types. In the brain, there is a reliance upon de novo serine synthesis as serine is known to have low permeability across the blood-brain barrier [36] and thus the proteins within this pathway are greatly expressed within this tissue type $[34,36]$. This is especially true during development. Patients that harbor mutations that decrease the activity of any of the serine synthesis enzymes present with neurological disorders [36]. This has been observed for all three enzymes and the extent of the neurological deficit's correlates to how much enzyme activity has been maintained. Mutations in PHGDH have been found in numerous patients and result in severe neurological issues [36]. Patients have also presented with mutations in PSAT1, which reduced activity to $15 \%$, and these patients also had severe neurological impairments [37].

As mentioned previously, increased flux through the SSP and increased expression of the SSP enzymes have been correlated with tumorigeneses in numerous cancer types [29]. The concept that serine biosynthesis and cancer are linked is not new. This was first introduced in the 1970s where it was found that there was increased growth in rat hepatoma cell lines that had higher PHGDH activity [38]. This was followed by work in the 1980s that found flux through the SSP was elevated and that this increase correlated with increased tumor growth [39]. In the years since these original findings, SSP enzymes have been established as crucial enzymes that are necessary for tumorigenesis in several different cancer types [29] and have been linked to therapy resistance and metastasis [28].

Much of this work has focused on the rate-limiting enzyme, PHGDH, as this 
would establish a metabolic requirement for general SSP function. However, there is evidence to support alternative roles for other enzymes within this pathway. Therefore, the rest of this dissertation will mainly focus on the relevance of the enzyme PSAT1. PSAT1 was first molecularly characterized in 2003 [40]. There are two different isoforms of PSAT1, PSAT1 $\alpha$ and PSAT1 $\beta$, that can be formed via alternative splicing [40]. Although there are two isoforms, only one, PSAT1 $\beta$, is presumed to be physiologically functional as it exhibits a greater level of expression compared to the $\alpha$ isoform [40]. The expression of PSAT1 depends on tissue type and cell cycle phase. PSAT1 is highly expressed in the brain, liver, kidney and pancreas and reaches maximum expression levels during S-phase [40]. As mentioned earlier, PSAT1 can also convert glutamate to $\alpha$-ketoglutarate. This gives PSAT1 a role in embryonic stem cell differentiation via control of $\alpha$ ketoglutarate levels [41] as it is estimated that PSAT1 accounts for $50 \%$ of the anaplerotic flux into the TCA cycle [27]. There has also been a substantial amount of evidence to support a role for PSAT1 specifically in cancer.

PSAT1 has been shown to be more active in neoplastic and proliferating tissues when compared to normal tissues [42]. This is due to lack of PSAT1 expression in many normal tissue types including lung, colorectal, ovarian and mammary tissues [34]. However, in tumors originating from these tissues expression of PSAT1 is greatly increased [43]. This upregulation of PSAT1 in these neoplastic cells has been linked to several different mechanisms, including epigenetic, miRNA, and transcriptional control.

Epigenetic regulation of PSAT1 has been shown to be a mechanism for 
increased serine synthesis. One epigenetic mechanism is reliant upon the interaction between menin and MLL1 which, when activated, maintains expression of the SSP enzymes in order to support proliferation, cell viability and tumor growth in Ewing Sarcoma [44]. Another epigenetic mechanism involves methylation at H3K9. In this context, euchromatic histone lysine methlytransferase 2 (G9A) adds an activating monomethylating mark, $\mathrm{H} 3 \mathrm{~K} 9 \mathrm{me} 1$, in order to drive transcription [45]. Conversely, KDM4C removes the repressive modification of $\mathrm{H} 3 \mathrm{~K} 9 \mathrm{me} 3$ which allows the transcription of this SSP enzyme [46]. Transcription following this epigenetic activation also requires activating transcription factor 4 (ATF4) [46].

This transcription factor has been shown to regulate PSAT1 in several different cancer types. In lung cancer, ATF4, under control of NRF2, regulates SSP enzyme expression in order to support glutathione and nucleotide production [47]. Also in lung cancer, ATF4 tethers MDM2 to the chromatin in order to drive activation of serine synthesis in order to support amino acid metabolism and redox homeostasis [48]. Lastly, ATF4 has been shown to regulate expression of PSAT1 in breast cancer to promote cell cycle progression [49].

There are also proteins other than ATF4 that have been implicated in regulating the serine synthesis pathway. The entire pathway is regulated by $p 73$ via glutaminase 2 (GLS-2) in lung cancer as a result of a need for converting glutamine into glutamate [50]. In Ewing Sarcoma, EWS-FLI1 drives SSP expression while increasing the uptake of glutamine in order to maintain redox homeostasis [51]. Along the same idea, SSP enzyme expression has been shown to be upregulated in response to different stress signals found in the tumor 
microenvironment. In liver cancer, glucose and glutamine deprivation resulted in a C-MYC-dependent increase in the enzymes of the SSP to support glutathione production, cell cycle progression, and nucleic acid synthesis [52]. Arginine deprivation was also shown to cause an upregulation in the SSP enzymes [53]. It has also been shown that serine deprivation causes an increase in the expression of the SSP enzymes to support cell survival by providing serine to maintain proliferation and mammalian target of rapamycin complex 1 (mTORC1) activity [54]. The SSP enzymes, including PSAT1, have also been shown to be induced by hypoxic conditions found in the tumor microenvironment [55].

PSAT1 can also be regulated independently from the other enzymes in serine synthesis pathway. PSAT1 has been shown to be a direct target of miR340, miR-365, and miR-424 [56-58]. This regulation occurs in both esophageal (miR-340 and miR-365) and colorectal cancer (miR-424). In every instance, the miRNA was acting as a tumor suppressor and loss of the miRNA resulted in an increase in PSAT1 expression and a subsequent increase in the tumorigenic properties of those cancer types [56-58].

Besides miRNA regulation, there are several other mechanisms implicated in the specific regulation of PSAT. In lung cancer, PSAT1 expression is controlled by the deactivation of NF1. The subsequent activation of FAK1 results in a glutamate dependence that is reliant upon PSAT1 [59]. In breast cancer two different means of regulation have been suggested for PSAT1. One proposed mechanism is through the transcription factors TAZ/YAP [60]. In this scenario TAZ/YAP induce PSAT1 expression to mediate a growth dependence on 
exogenous glutamine [60] and this is primarily observed in high grade TNBCs $[60,61]$. The other proposed mechanism in breast cancer is regulation by the $\mathrm{PI} 3 \mathrm{~K} / \mathrm{Akt} / \mathrm{mTOR}$ pathway [62]. Control of PSAT1 in this pathway was achieved via a reduction of mRNA and protein levels of PSAT1 following mTOR inhibition and MyrAkt induction was able to attenuate this reduction in expression [62]. It was also determined that the regulation of PSAT1 by the PI3K/Akt/mTOR pathway was independent of proliferation [62]. Induction of expression of PSAT1 by mTORC1 was also observed in pancreatic cancer, but in that context, it seemed to be a means of SSP regulation and not PSAT1 specifically [63]. These various mechanisms utilized for the regulation of PSAT1 demonstrate a need for the expression of this protein in cancer cells and suggests that PSAT1 plays a crucial role in malignant disease.

As noted, PSAT1 has been demonstrated to have increased expression in several types of cancer. This upregulated expression appears to be directly related to survival in most of these cancer types. Its expression has been correlated with increased proliferation, resistance to therapy, metastasis, and poorer patient outcomes. The following section will focus on what role, if known, PSAT1 plays in various cancer types and if these roles are related to the SSP or an independent function of PSAT1.

\section{PSAT1 in Thyroid Cancer}

In thyroid cancer, the serine synthesis pathway is activated and requires PSAT1 activity. It was found that the expression levels of the SSP enzymes could determine the subtype of thyroid cancer, as the SSP enzymes had higher 
expression in the poorly differentiated carcinoma (PDC) and papillary thyroid carcinoma (PTC). Yet, they had very low expression in the medullary carcinoma (MC) subtype [64]. This group also found that a BRAF mutation (V600E) associated with PSAT1 expression. Patients with this BRAF mutation had higher expression of the SSP enzymes compared to patients with wild-type BRAF [64].

\section{PSAT1 in Nasopharyngeal Carcinoma}

In nasopharyngeal carcinoma, PSAT1 was identified as a potential biomarker for prognosis [65]. In this cancer type, high PSAT1 expression associated with an aggressive clinical course and advanced tumor stage. In addition, it correlated with reduced disease-specific survival, distant-metastaticfree survival, local-recurrence-free survival, overall survival, and was an overall indicator of poorer patient prognosis [65].

\section{PSAT1 in Melanoma}

The effects of PSAT1 in melanoma are directly related to its function regarding serine biosynthesis. Melanoma has one of the highest frequencies of PHGDH amplifications compared to other cancer types [66]. This amplification of

PHGDH, along with increased expression of the other SSP enzymes, results in increased flux through the serine biosynthetic pathway [67]. Cells that harbor these PHGDH amplifications are sensitive to PHGDH, or other SSP enzyme, suppression where silencing leads to a reduction in cell proliferation [67]. These SSP enzymes, including PSAT1, have also been identified as necessary for the observed resistance to BRAF inhibitors in this cancer type [68].

PSAT1 in Pancreatic Cancer 
The role of PSAT1 in pancreatic cancer is also related to its function in the serine synthesis pathway. The SSP enzymes in pancreatic cancer can be induced by mTOR [63]. This overexpression is required for further down-stream one-carbon metabolism that supplies the s-adenosylmethionine levels in order to meet the methylation needs of the cell [63]. The overexpression of PSAT1 is also required for tumorigenesis of pancreatic cancer, especially under the conditions of liver kinase B1 (LKB1) loss. Upon loss of LKB1, PSAT1 is required for proliferation, colony formation, and subcutaneous tumor growth [63]. SSP enzymes have also been identified as necessary components of BRAF inhibitor resistance [68]. In resistant cell types, the BRAF inhibitor vemurafenib induced the expression of the SSP enzymes and resulted in an increase in cell proliferation [68].

\section{PSAT1 in Multiple Myeloma}

The expression of PSAT1, and other serine synthesis enzymes, in multiple myeloma has been directly associated with resistance to therapeutic treatment [69]. It was observed that all SSP enzymes had higher expression in cell types that were determined to be resistant to bortezomib [69]. This correlation was so significant that it was determined that SSP enzyme expression could potentially be used as a biomarker to identify patients that would be resistant to this therapeutic option [69].

\section{PSAT1 in Hepatocellular Carcinoma}

The role of PSAT1 in normal liver differs from other tissues and this also extends to tumors originating from this tissue type. In hepatocellular carcinoma, $\mathrm{PSPH}$, the rate-limiting enzyme in the liver, is significantly upregulated compared 
to normal liver expression while PHGDH and PSAT1 are downregulated [70]. It is suggested that these expressions patterns are due to a developed reliance on glutamine within the tumor in order to fuel the TCA cycle [70].

\section{PSAT1 in Glioblastoma}

With the dependence in the brain on de novo serine synthesis, these proteins are normally expressed at higher levels than other tissues [34]. In glioblastoma, there is a reduction in the expression of PSAT1 in tumors compared to normal tissue and this reduction in expression is exacerbated in higher grade tumors compared to lower grade tumors [71]. It was also determined that certain therapies, i.e. Regorafenib, require high PSAT1 expression in order to suppress tumor growth in glioblastoma multiforme [71].

\section{PSAT1 in Ewing Sarcoma}

In Ewing sarcoma, the entire serine synthesis pathway is highly active, and these enzymes (PHGDH, PSAT1, and PSPH) are essential for proliferation, viability, and tumor growth [44]. This pathway has also been associated with regulation of redox homeostasis and protecting these tumors against DNA damage

and apoptosis [51]. The expression of the SSP enzymes are also an indicator of prognosis within this cancer type. Patients with higher expression are classified as a high-risk group with poorer overall survival [51].

\section{PSAT1 in Esophageal Squamous Cell Carcinoma}

PSAT1 expression is elevated in esophageal squamous cell carcinoma compared to normal tissue [72]. Characteristically, elevated PSAT1 expression contributes to proliferation, epithelial to mesenchymal transition, invasion, and 
progression $[57,58,72]$. Higher expression of PSAT1 also enhanced tumor formation in vivo [72]. Functionally, it is suggested that PSAT1 is contributing to these tumorigenic processes via a promotion of GSK3ß/snail activity [72]. Clinically, increased expression of PSAT1 has been correlated with stage of disease, lymph node metastasis, distant metastasis, and overall poorer patient prognosis [72].

\section{PSAT1 in Colon Cancer}

PSAT1 is highly expressed in colon tumor tissue compared to normal colon $[73,74]$ and has even been identified as the most upregulated gene in colorectal carcinoma [75]. Increased PSAT1 expression in colon cancer has been shown to have an integral role in tumor growth both in vitro and in vivo as well as evasion of apoptosis [56]. Clinically, higher levels of PSAT1 expression has been associated with chemo-resistance $[73,75]$. Increased PSAT1 expression correlates with a reduced sensitivity to oxaliplatin [73] as well as resistance to irinotecan, 5fluorouracil, and leucovorin therapy and a resulting decrease in patient survival time [75]. This finding has led to the suggestion that PSAT1 be used as a biomarker for resistant colorectal carcinoma [75]. Mechanistically, it seems that PSAT1 is contributing to an increased growth rate and chemo-resistance via a canonical function in serine-synthesis and downstream one-carbon metabolism [76].

\section{PSAT1 in Ovarian Cancer}

In ovarian cancer, PSAT1 expression is increased in tumor tissue compared to adjacent normal tissue [77-79]. This overexpression can be used as a biomarker to sort into different molecular categories as PSAT1 was observed to be strongly 
expressed in the ovarian clear cell carcinoma subtype [78]. It has also been found that PSAT1 expression levels correlate to clinical stage and tissue differentiation [79]. Elevated PSAT1 expression contributes to disease occurrence, development, and prognosis [79] via a promotion of GSK3 $\beta$ phosphorylation, angiogenesis, and enhanced expression of HIF-1a, VEGF, and $\beta$-catenin [77]. In addition, elevated PSAT1 expression in ovarian cancer has been linked to resistance to cisplatin treatment [77].

\section{PSAT1 in Lung Cancer}

In lung cancer there is evidence for both SSP and independent roles of PSAT1. The function of PSAT1 in lung cancer that is related to serine biosynthesis is driven by a need for flux through the pathway in order to generate serine. This supports cell proliferation and mTORC1 activity during times of serine deprivation [54]. This also occurs as a response to oxidative stress [50] as a means to regulate redox homeostasis during these conditions [48]. Reliance upon the SSP has also been defined as a specific molecular subtype in lung adenocarcinoma. The enzymes in the SSP are upregulated in this pathway in order to maintain flux through the serine pathway and correlates with significantly poorer patient prognosis [80]. In addition, PSAT1 was found to be significantly upregulated in non-small cell lung cancer [81]. This increase contributed to cell cycle progression, cell proliferation, tumorigenesis, and was involved in the regulation of the Rb-E2F pathway activity by inhibition of cyclin D1 degradation [81]. This suggests that PSAT1 may have a function outside of its enzymatic activity in contributing to generation of serine. In addition, clinically overexpressed PSAT1 conferred a 
poorer clinical outcome for patients [81] PSAT1 was also identified as mediating a glutamine dependence in a subset of Kras driven lung adenocarcinomas that harbor a loss of function mutation in NF1 via a FAK1-dependent mechanism [59]. PSAT1 in Breast Cancer

A connection between PSAT1 expression and breast cancer was first described in 2005 by Martens et al. They found that the extent of methylation on the PSAT1 promoter, and thus PSAT1 mRNA expression, was indicative of a response to tamoxifen therapy in ER+BC with recurrent disease [82]. Since then, there has been an increase in the investigation into the role of the serine synthesis pathway and PSAT1 in the development, progression, and treatment of breast cancer. Most of this work has looked at PSAT1 in the context of its canonical function within the serine synthesis pathway.

It has been determined that serine synthesis pathway enzyme expression levels in breast cancer could predict patient survival [83]. The enzymes in the SSP were upregulated in a metastatic variant of TNBC breast cancer and that this increase was correlated with decreased relapse-free and overall survival of patients with TNBC [84]. It was also noted in these studies that increased serine biosynthesis promoted bone metastasis via the stimulatory effect of serine on osteoclastogenesis [84] and that PSAT1 expression specifically was required for this development [85]. Certain breast cancers exhibit a dependence on flux through the serine synthesis pathway, driven by a dependence on PHGDH as the rate-limiting enzyme. In these subsets, SSP enzyme expression is required for tumorigenesis and correlates with aggressiveness of disease [86-88]. This 
requirement for flux through the SSP, also termed serine synthesis-dependence, makes these cells sensitive to manipulation in SSP enzyme expression wherein either genetic reduction or pharmaceutical inhibition causes a significant proliferative defect in these cell types $[86,87,89]$. As was mentioned earlier, hypoxia can induce expression of the SSP enzymes. This hypoxic induction of the SSP enzymes allows for the maintenance of redox homeostasis as serine synthesis reactions generate NADPH [90]. This causes an enrichment in breast cancer stem cells and indicates a role for this pathway in the evasion of cell death from chemotherapy treatment and in the formation of secondary tumor sites $[55,91]$. The increased expression of the SSP enzymes are not restricted to tumor cells. It has also been showed that there is an increase in SSP enzymes in stromal tissue that correlates with an increase in histological grade of breast phyllode tumors [92]. In addition, there is evidence to support that depending on the origination of the disease, i.e. ductal or lobular, expression of the SSP enzymes will be higher in either the tumor cells (invasive lobular carcinoma) or stromal tissue (invasive ductal carcinoma) [93].

Outside of the serine synthesis pathway in general, there has been investigation into the role of PSAT1 in breast cancer. PSAT1 was shown to have higher expression in estrogen receptor negative cancers compared to estrogen receptor positive cancers due to a loss of promoter methylation [94]. In addition to ER status, PSAT1 promoter methylation (i.e. lack of PSAT1 expression) correlated with low-grade, low-proliferation, lymph node positive BC in women who were postmenopausal and Caucasian [95]. Low promoter methylation of PSAT1 (i.e. high 
PSAT1 expression) was associated with higher tumor grade, $\mathrm{pN}$ stage, and poorer disease-free survival in patients [96]. As mentioned earlier, PSAT1 mRNA levels were able to be a predictor of response to tamoxifen treatment [82]. That work was further expounded upon and demonstrated that protein levels of PSAT1 were also associated with poorer patient outcome upon tamoxifen treatment [97]. Further, high PSAT1 expression has been correlated to poorer overall survival, recurrence free survival, and identified as part of a gene signature inclusive of all breast cancer subtypes [98].

While it is evident that there is an increase in the expression of PSAT1 in several cancer types and that increased PSAT1 generally associates with clinical outcomes, I will be focusing the rest of this dissertation on the role(s) of PSAT1 in breast cancer. It is known that PSAT1 is increased in breast cancers and that this increase correlates with poorer survival for patients; however, the requirement of PSAT1 in different subtypes of breast cancer is still poorly understood in the progression of this disease. While there is a clear indication that there is a role for PSAT1's function within the serine synthesis pathway in breast cancers that are serine synthesis-dependent, this only accounts for a limited subset of breast cancers. In cancers that are classified as independent of serine synthesis, yet still express elevated levels of PSAT1, it is unclear what function or role increased PSAT1 provides the tumor in this context. The majority of this doctoral work has been completed in the context of TNBC as the expression of PSAT1 is greater in this molecular subtype even in those TNBCs designated as serine synthesis independent. However, PSAT1 has also been reported to be expressed in ER+BC, 
especially in tumors that are resistant to endocrine therapy. To date, there is no indication of whether this therapy induced increase in expression promotes either elevated flux through the serine pathway or a non-canonical function of PSAT1 that contributes to acquired resistance.

These gaps in knowledge are the basis for this dissertation. The role of PSAT1 in a serine synthesis-independent system will be examined in TNBC. The rationale for investigation in this molecular subtype is that while a large portion of TNBCs $(70 \%)$ upregulate the serine biosynthetic enzymes, only $6 \%$ of that $70 \%$ are due to genetic amplification of PHGDH [87]. These statistics suggest that there is a large subset that may upregulate PSAT1 independently of other SSP enzymes and thus the function for this increase is unknown. Due to the metastatic nature of TNBCs, the variable independent increases observed in PSAT1, PSAT1's correlation with higher grade TNBCs, and the association between higher grade tumors and metastasis, we speculate that PSAT1 may be involved in the metastatic potential of TNBC (Aim 1) and this function of PSAT1 is unrelated to its function in serine biosynthesis (Aim 3).

The other aim (Aim 2) of this dissertation is to further investigate a finding by Martens et al. High expression of PSAT1 was demonstrated as the strongest negative correlation with response to endocrine therapies in a study on patients with recurrent ER+ breast cancer [82]. In a follow-up study these results were confirmed at the protein level and in a second patient cohort [97]. This second study suggests that the contribution of PSAT1 to resistance may rely on flux through the serine synthesis pathway as increases in PHGDH were also observed. 
It is my goal to examine the effects of altered PSAT1 expression on the sensitivity to endocrine therapy in both endocrine sensitive and resistant ER+BC. In addition, we will examine whether these suggested functions of PSAT1 are the result of its involvement in the serine biosynthetic pathway.

The elevated expression of PSAT1 in breast cancer compared to normal mammary tissue, combined with previously published data and our own results suggests that PSAT1 contributes to breast cancer progression by promoting TNBC metastasis in a serine-independent context and endocrine resistance in ER+BC possibly through a requirement for serine synthesis. I have devised three aims to evaluate this hypothesis.

1. Determine the effect of PSAT1 suppression in breast cancer metastasis in vitro and in vivo. This aim will be evaluated in a specific subset of TNBC that is "serine synthesis-independent" and will implement several in vitro assays of metastatic characteristics.

2. Determine the effect of PSAT1 expression on endocrine resistance within ER+BC. This aim will be evaluated in both ER+ endocrine sensitive and endocrine resistant cell lines and determine if suppression of PSAT1 affects sensitivity to endocrine therapies.

3. Determine if there is a requirement for serine synthesis and thus PSAT1 in breast cancer progression. The requirement for serine synthesis will be evaluated in our TNBC model system and employ both the suppression of PSAT1 as well as the suppression of the rate-limiting enzyme PHGDH. 


\section{CHAPTER 2}

SELECTIVE LOSS OF PHOSPHOSERINE AMINOTRANSFERASE 1 (PSAT1)

\section{SUPPRESSES TRIPLE NEGATIVE BREAST CANCER METASTASIS}

\section{Introduction}

Breast cancer is the most prevalent cancer diagnosis and the second leading cause of cancer-related deaths among women. Metastatic disease is responsible for over $90 \%$ of breast cancer related mortalities [15], which culminates in an overall survival rate of $22 \%$ for metastatic patients [2]. Metastasis or advanced recurrent disease affects approximately $30 \%$ of patients and is clinically challenging as therapies are often directed at prolonging survival without compromising quality of life [14]. Metastasis can arise from all breast cancer subtypes [17] but triple negative breast cancer (TNBC) is considered the most aggressive as it correlates with poorer overall survival in both early stage and metastatic disease [4]. Separate from hormone receptor positive breast cancers, TNBC is characterized by the lack of estrogen receptor- $\alpha(E R \alpha)$, progesterone receptor (PR), and human epidermal growth factor receptor 2 (HER2) expression [4].

Despite the inherent heterogeneity of TNBC tumors, $70 \%$ of TNBCs upregulate the expression of enzymes within the serine synthesis pathway (SSP) [87]. The SSP is a glycolytic shunt that is responsible for converting 3- 
phosphoglycerate into serine through a series of three reactions. The first reaction, and rate-limiting step, is catalyzed by phosphoglycerate dehydrogenase (PHGDH) and converts 3-phosphoglycerate into 3-phosphohydroxypyruvate. The second enzyme, phosphoserine aminotransferase (PSAT1), couples the conversion of glutamate to a-ketoglutarate with the production of 3-phosphoserine. Phosphoserine phosphatase (PSPH) then dephosphorylates 3-phosphoserine into serine. Most of the previous work involving the SSP and TNBC has focused on a role for PHGDH. Select primary TNBCs and TNBC cell lines have been previously designated as "serine-dependent" or reliant on the cellular production of serine. These cells harbor genetic amplifications of PHGDH or have extremely high PHGDH expression, within which PHGDH silencing causes a significant decrease in proliferation due to loss of alpha-ketoglutarate production through PSAT1 [87]. However, these gene amplifications of PHGDH only account for $6 \%$ of primary tumors and $18 \%$ of breast cancer cell lines [87]. "Serine synthesis-independent" TNBC cell lines do not harbor genetic amplifications of PHGDH and loss of SSP enzymes yields no anti-proliferative effect in the presence of exogenous serine. Yet, many of these cell types demonstrate elevated expression of the SSP enzymes compared to normal breast tissue, particularly PSAT1, suggesting that these enzymes may have additional functions in TNBC progression $[55,87]$.

While PSAT1 is not expressed in normal mammary tissue [34], it is elevated in several breast cancer subtypes as well as in several other aggressive malignancies including colon cancer, nasopharyngeal carcinoma, human esophageal squamous cell carcinoma, and non-small cell lung cancer 
$[49,55,65,72,73,81]$. Control of PSAT1 expression is driven by several oncogenic pathways and is differently regulated throughout cell-cycle progression $[42,44,47,49,50,52,60]$. Increased PSAT1 expression has been correlated with multiple tumorigenic characteristics, including metastasis and chemoresistance, and is correlated with poorer patient prognosis $[49,72,73,81,84]$.

Based on previous reports detailing the requirement for SSP enzymes in "serine synthesis-dependent" TNBC proliferation coupled with PSAT1's elevated expression in "serine synthesis-independent" TNBC cells, we examined PSAT1's potential contribution to other TNBC features, particularly in metastatic potential. In this report, we demonstrate that PSAT1 expression increases with TNBC grade and that selective suppression of PSAT1 inhibits metastatic characteristics both in vitro and in vivo without affecting proliferation. Importantly, loss of PHGDH in "serine synthesis-independent" cells did not phenocopy PSAT1 silencing, suggesting that these effects are specific to PSAT1 in these cell types. Together, these data indicate that PSAT1 plays a role in metastatic potential in TNBC. 


\section{Methods and Materials}

\section{Immunohistochemistry}

PSAT1 expression was determined in a panel of commercially available tissue microarrays (TMA) that contained de-identified TNBC tumor core sections with provided clinical characteristics, such as grade and histological status of the estrogen and progesterone receptors and HER2 (US Biomax, BR487, BR243, BR1503, and BR1504). TMAs were dewaxed and rehydrated using a series of xylene and ethanol washes. Tissues were then blocked with 5\% goat serum and incubated with 1:100 dilution of anti-PSAT1 antibody (ProteinTech) for 16 hours at $4^{\circ}$. Sections were subsequently incubated with 1:1000-fold dilution of HRPconjugated anti-rabbit antibody. PSAT1 was detected using DAB stain (Vector Laboratories), counterstained with hematoxylin, and image was digitally captured using AperioScope digital slide scanner.

Analysis of PSAT1 staining in TNBC sections (ER-, PR-, and HER2staining as defined by the provided histological reports with each TMA) was performed using Aperio ImageScope software with the positive pixel count algorithm. Areas of analysis excluded stromal tissue in the tumor samples and was defined to ductal epithelial in the normal breast tissue sections. Comparison of relative PSAT1 levels between normal, grade 1, grade 2, and grade 3 TNBC tissues was determined by dividing the positive pixel count by total pixels (sum of positive and negative) as determined by the software.

\section{Cell Culture}


The MDA-MB-231 and HCC1806 TNBC cell lines were obtained from ATCC. MDA-MB-231 cells were cultured in IMEM supplemented with 5\% FBS and gentamicin and HCC1806 cells were cultured in RPMI-1640 medium supplemented with $10 \%$ FBS and gentamicin. HEK 293 T cells were also obtained from ATCC and cultured in DMEM supplemented with 10\% FBS and gentamicin. All cells were incubated at $37^{\circ}$ in $5 \% \mathrm{CO}_{2}$.

\section{Plasmid Generation}

The PSAT1 and TIMP2 genes were isolated via the utilization of primers targeting the 3 ' and 5' ends of the mature RNA encoding for either PSAT1 or TIMP2. These primers were adapted to include the restriction enzymes ECOR1 and BAMH1. Polymerase chain reactions were performed to amplify the region and the products were then separated via gel electrophoresis. The corresponding bands were excised from the gel and purified. They were then ligated into either a FLAG-tagged vector (PSAT1) or a GFP-tagged vector (TIMP2), transformed into bacterial strains, and plated on LB agar with ampicillin. A single bacterial colony was then cultured in LB broth and ampicillin for 24 hours and plasmids were isolated from the culture via the Qiagen Miniprep kits and confirmed by sequencing.

\section{Transfections}

PSAT1 siRNA and scrambled negative control siRNA were purchased from Ambion. PHGDH siRNA and non-targeting control pool siRNA were purchased from Dharmacon. PSAT1 shRNA, PHGDH shRNA, and Control shRNA were purchased from Sigma. The FLAG-PSAT1 and GFP-TIMP2 were generated as 
described above. For overexpression, FLAG-PSAT1, GFP-TIMP2, and the empty vectors were transfected into HEK 293 cells using the Polyplus jetPRIME reagent. For transient suppression, siRNAs were transfected into either MDA-MB-231 or HCC1806 cells using RNAiMax Lipofectamine or Polyplus INTERFERin systems according to manufacturer's protocol. For stable suppression cell line generation, shRNAs were transfected into cells using the Polyplus jetPRIME reagent according to manufacturer's protocol and clonal selection for gene silencing was performed using puromycin. The sequences for all siRNA and shRNA species are as follows: PSAT1 RNAi: 5'-CCCUAAACUUGGGAGUUAUtt-3', negative control RNAi: Silencer Select Negative Control No. 2 cat\#4390846, PHGDH RNAi: 5'CGACAGGUUGCUGAAUGA-3', non-targeting pool RNAis: 5'UGGUUUACAUGUCGACUAA-3', 5'-UGGUUUACAUGUUGUGUA-3', 5''UGGUUUACAUGUUUUCUGA-3', 5'-UGGUUUACAUGUUUUCCUA-3', PSAT1 ShRNA: $\quad$ 5'CCGGGCACTCAGTGTTGTTAGAGATCTCGAGATCTCTAACAACACTGAGTG $\begin{array}{llll}\text { CTTTTTG-3', } & \text { PHGDH } & \text { 5'- }\end{array}$ CCGGCTTCGATGAAGGACGGCAAATCTCGAGATTTGCCGTCCTTCATCGAA GTTTTTG-3', Control shRNA: CCGGCAACAAGATGAAGAGCACCAACTCGAGTTGGTGCTCTTCATCTTGTT GTTTTT-3'.

\section{Immunoblot Assays}

Whole-cell lysates were prepared in IP Lysis Buffer (Pierce) containing protease and phosphatase inhibitors. Proteins were separated on $10 \%$ SDS- 
polyacrylamide gels and transferred to PVDF. Membranes were blocked with 5\% non-fat dry milk in TBS-T and subsequently probed with 1:1000-fold dilution of antiPSAT1 (Proteintech, 10501-1-AP) or anti-PHGDH (Sigma HPA021241) antibodies for 16 hours at $4^{\circ}$. Washed membranes were then incubated with 1:5000 dilution of HRP-conjugated anti-rabbit or anti-mouse secondary antibodies. Protein detection was done by exposure to ECL Prime chemiluminescent reagent (GE Healthcare). Protein loading was assessed using anti- $\beta$-actin antibody (Sigma, A2228).

\section{Cell Proliferation}

For shRNA silencing, MDA-MB-231 $(50,000)$ cells with or without stable knock-down of PSAT1 or PHGHD were seeded in triplicate in a 12-well (Corning) plate. Cell proliferation was assessed by counting of trypan blue excluded cells 24 and 48-hours post-seeding. For siRNA suppression, MDA-MB-231 $(50,000)$ or HCC1806 $(30,000)$ were seeded in 12-well or 24-well (Corning) plate respectively, 24-hours post-transfection. Cells were then counted via trypan blue exclusion 24 or 48 -hours post-seeding.

\section{Wound Healing Assay}

MDA-MB-231 or HCC1806 cells were seeded at 200,000 cells per well in a 12-well (Corning) plate. Cells were either seeded directly (stable shRNA suppression) or 24-hours post siRNA transfection. Scratch in the confluent monolayer was generated with a sterile $200 \mu \mathrm{L}$ pipette tip. Images were taken at OHR and $24 \mathrm{HR}$ time intervals and \% wound healing was quantified using ImageJ.

\section{Migration and Invasion Assays}


For migration assays, MDA-MB-231 and HCC1806 cells were serum starved for 24 hours and then plated (25,000 and 50,000 respectively) into Boyden chambers in serum-free medium. Serum-containing medium was placed in each well to serve as the chemoattractant. At 24 hours, inserts were fixed with $100 \%$ methanol for 10 minutes and non-migrated cell were removed with a cotton swab. The inserts were then washed with PBS and stained with crystal violet. Images were captured via an EVOS microscope and multiple 4X fields were analyzed with ImageJ.

For invasion assays, MDA-MB-231 cells were serum starved for a period of 24 hours and then plated $(50,000)$ into Corning Biocoat Matrigel Invasion Chambers (Corning) following rehydration according to manufacturer's protocol. The cells were allowed to invade for 24 hours at which point the inserts were fixed with $100 \%$ methanol for 10 minutes and non-invasive cells and remaining matrigel layer were removed via cotton swab. The inserts were then washed with PBS and stained with crystal violet. Images were captured via an EVOS microscope and multiple 4X fields were analyzed with ImageJ.

\section{Quantitative-Real Time Polymerase-Chain Reaction}

MDA-MB-231 or HCC1806 cells were collected and total RNA was isolated using the RNeasy Mini Kit (Qiagen). One milligram of RNA was converted to cDNA using the High-Capacity RNA-to-cDNA kit (appliedbiosystems) according to the manufacture's protocol. Samples were then analyzed for qPCR via the TaqMan Fast Advanced (appliedbiosystems) system with human probes for PSAT1 (Hs00795278_mH), PHGDH (Hs00198333_m1), and ACTB (Hs01060665_g1). 


\section{Anchorage-Independent Growth}

A bottom layer of $0.6 \%$ noble agar in complete medium was prepared in sixcentimeter dishes. MDA-MB-231 cells were seeded at 50,000 cells per dish in a $0.3 \%$ agar solution. Cell colonies were refreshed with $0.25 \%$ agar/medium solution every 3-5 days during the 21-day assay. Images were captured via a Nikon digital camera (DXM1200F) attached to a Nikon (SMZ1500) microscope.

\section{Actin Cytoskeleton Staining}

MDA-MB-231 $(30,000)$ or HCC1806 $(60,000)$ cells were plated in Lab-Tek II Chamber slides (154526) either directly or following transfection with siRNA species. Forty-eight hours after plating, cells were fixed with $3.7 \%$ formaldehyde solution. The cells were then permeabilized with $0.1 \%$ Triton X-100 in PBS before staining with phalloidin (Thermo Fisher) and DAPI (Thermo Fisher) following manufacturer's protocol. Cellular staining was visualized using an Olympus FV3000 confocal microscope equipped with Flouview software (Olympus America Inc.) under 40X magnification.

\section{Animal Model for Experimental Metastasis}

The in vivo study was approved by the University of Louisville's Institutional Animal Care and Use Committee. The experimental in vivo metastasis model was performed as previously established for MDA-MB-231 cells [99]. Briefly, MDA-MB231 cells with or without PSAT1 silencing were collected, washed, and resuspended in PBS at a concentration of $1 \times 10^{6} / \mathrm{mL}$. One hundred microliters of cell suspension were intravenously injected into the tail vein of athymic nude mice (Charles River). Lung tissue was resected 8 weeks post-injection, formalin fixed, 
and embedded in paraffin. FFPE lung tissue was sectioned and stained with hematoxylin and eosin to detect MDA-MB-231 lung lesions. Images were captured using AperioScope digital slide scanner and micro-metastatic foci were counted from three separate image fields (under $4 \mathrm{X}$ magnification) across individual tissue sections from each animal.

\section{Co-Immunoprecipitation}

HEK 293T cells were transfected with FLAG-PSAT1 and GFP-TIMP2. Forty-eight hours post transfection, cell pellets were collected and whole-cell lysates were prepared in IP Lysis Buffer (Pierce) containing protease and phosphatase inhibitors. Anti-FLAG M2 magnetic beads (Sigma) were prepared according to manufactures instructions prior to overnight incubation with $1 \mathrm{mg}$ of protein lysate. Proteins not bound to the beads were removed by washing prior to elution. Remaining proteins were separated on 10\% SDS-polyacrylamide gels and transferred to PVDF. Membranes were blocked with 5\% non-fat dry milk in TBS-T and subsequently probed with 1:1000-fold dilution of anti-FLAG (DYKDDDDK) (Cell Signaling, 23685), anti-PSAT1 (Proteintech 10501-1-AP), or anti-GFP (Sigma G1546) antibodies for 16 hours at $4^{\circ}$. Washed membranes were then incubated with 1:5000 dilution of HRP-conjugated anti-rabbit or anti-mouse secondary antibodies. Protein detection was done by exposure to ECL Prime chemiluminescent reagent (GE Healthcare).

\section{Statistical Analysis}

Statistical analysis of all studies was performed with Graph Pad Prism software using either analysis of variance or unpaired t test. $p$ values for all results 
are indicated in their respective figure legends. 


\section{Results}

\section{PSAT1 expression increases with TNBC grade}

In support of the clinical relevance of the serine synthetic pathway in TNBC, we performed immunohistochemistry analysis of breast tissue samples from normal tissue, or at varying grades of TNBC. Similar to previous reports [34], we found that normal breast tissues express low levels of PSAT1 compared to TNBC (Figure 5A). Quantitation of PSAT1 staining revealed a trend towards elevated levels in grade 1 tumor tissue that is significantly increased in higher grades within these TNBC tissues (Figure 5B). These results demonstrate that PSAT1 is expressed in breast tumor tissue, suggesting that PSAT1 has a potential role in the progression of TNBC.

\section{Suppression of PSAT1 inhibits migration and invasion of select TNBC cell} types without affecting cell proliferation

Prior studies have established the SSP as a crucial pathway in cancer progression, including TNBC $[84,87]$. However, most of this work has focused on a subset of TNBC designated as "serine-synthesis dependent" in which suppression of any of the SSP enzymes causes a significant proliferative defect. However, there are cell lines in which serine synthesis is dispensable for cell proliferation; yet they still upregulate SSP enzymes when compared to normal breast tissue [87]. This increased expression of SSP enzymes, namely PSAT1, in these serine synthesis-independent cell lines suggests that they may promote tumorigenic properties beyond enhanced proliferation. Based on this, we sought to determine if there was a metastatic role for PSAT1 in two defined serin 
A
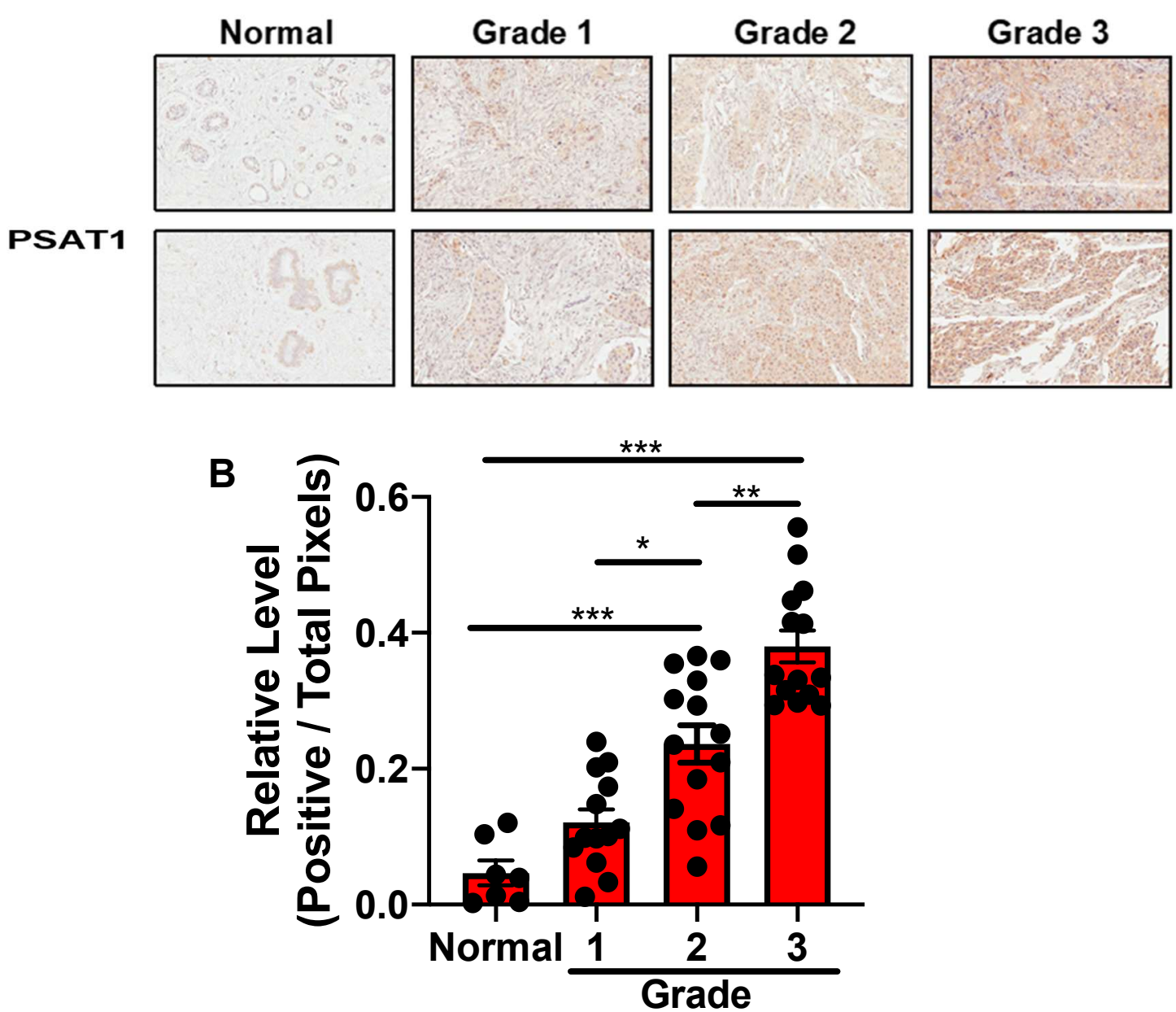

Figure 5: PSAT1 expression increases with TNBC grade. A) Formalin-fixed paraffin embedded tissue sections were examined by immunohistological staining for PSAT1. Shown are two independent representative images (20X) from either normal breast tissue or from different grades of TNBC from tissue microarray core sections. B) Quantitation of PSAT1 staining demonstrates a significant increase in expression in higher TNBC grade. Relative levels of PSAT1 was determined from multiple normal $(n=7)$, grade $1(n=11)$, grade 2 $(n=14)$, and grade $3(n=14)$ independent TMA tissue sections. Quantification is demonstrated as relative level (positive / total pixel) of PSAT1 expression and shown are mean \pm SEM. ${ }^{*} p<0.01,{ }^{* *} p<0.005,{ }^{* * *} p<0.0001$. 
synthesis-independent TNBC cell models, MDA-MB-231 and HCC1806 [87]. We first found differential expression of PHGDH and PSAT1 in both cell types. While MDA-MB-231 cells predominantly expressed PSAT1 compared to low level of PHGDH, HCC1806 cells exhibited similar levels of both SSP enzymes (Figure 6). Consistent with previous reports [55], we found that loss of PSAT1, via either stable shRNA or transient siRNA silencing, did not negatively affect cell proliferation (Figure 7A\&B, Figure 8A\&B). Alteration in cell morphology, in part due to changes in actin cytoskeleton rearrangement, can directly influence the motile capability of tumor cells. To examine whether loss of PSAT1 affected cytoskeleton structure and cell morphology, we performed phalloidin staining to visualize the F-actin cytoskeleton structure. Upon suppression of PSAT1, MDAMB-231 cells exhibited changes in both F-actin cytoskeleton arrangement and overall cell morphology compared to control cells (Figure 7C). In particular, there was a reduction in the spindle-shaped morphology normally observed in these TNBC cells and a disruption in the actin stress fibers (Figure 7C). These results suggest that PSAT1 may be important for maintenance of cell morphology and may contribute to other tumorigenic properties in the MDA-MB-231 cells, particularly cell motility. To determine the relevance of PSAT1 loss on this cellular function, we examined the migratory ability of MDA-MB-231 cells, by both wound healing and Boyden chamber transwell assays. Both stable and transient suppression of PSAT1 significantly decreased motility of MDA-MB-231 cells by $>50 \%$ in both experimental procedures (Figure 9A\&B, Figure 10A\&B). We next investigated the invasive potential of those cells using the matrigel coated 

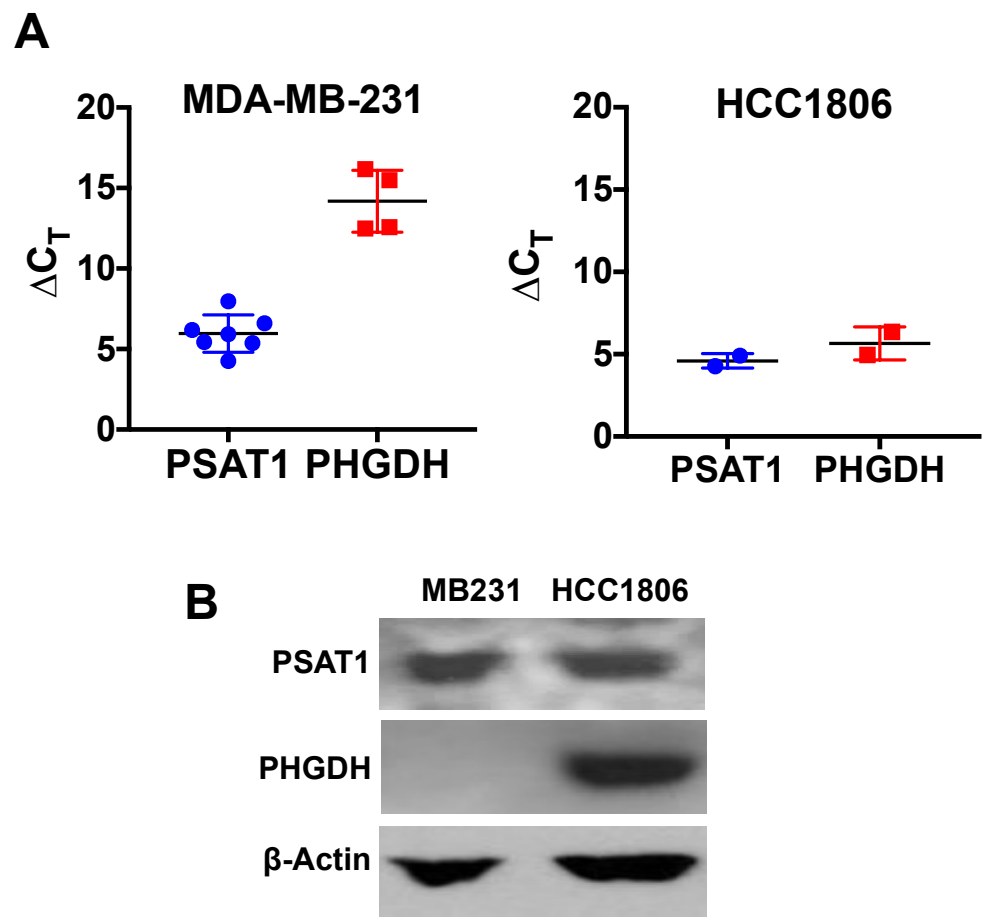

Figure 6: PSAT1 and PHGDH transcript and protein expression in MDA-MB-231 and HCC1806 TNBC cell lines. A) Transcript levels of PSAT1 and PHGDH was determined by RT-PCR. Data is presented as change in $\mathrm{C}_{\mathrm{T}}$ values between PSAT1 or PHGDH and $\beta$-actin (mean \pm SD). B) Western analysis of PSAT1 and PHGDH expression in both the MDA-MB-231 and HCC1806 cell lines. 

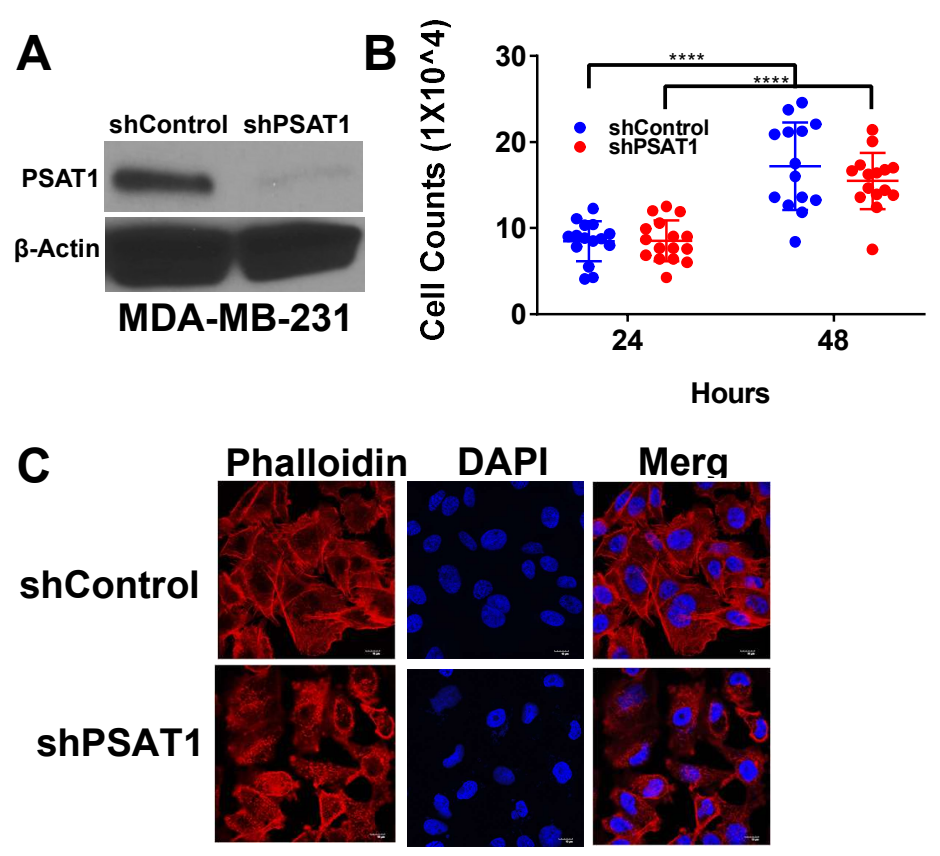

Figure 7: Loss of PSAT1 does not affect proliferation but alters appearance actin-cytoskeleton. A) Western blot analysis of PSAT1 expression in MDA-MB-231 cells selected for stable expression of control or PSAT1-specific shRNA. B) Cell proliferation in control or PSAT1-shRNA MDAMB-231 cells. Data are represented as mean \pm SD of viable cell counts at 24 and 48 hours. Results were analyzed by 2-way ANOVA, p<0.0001 C) Representative images of cytoskeleton F-actin filaments upon phalloidin staining in both control and PSAT1 shRNA MDA-MB-231 cells. Note: Control is the same in the PHGDH experiments 


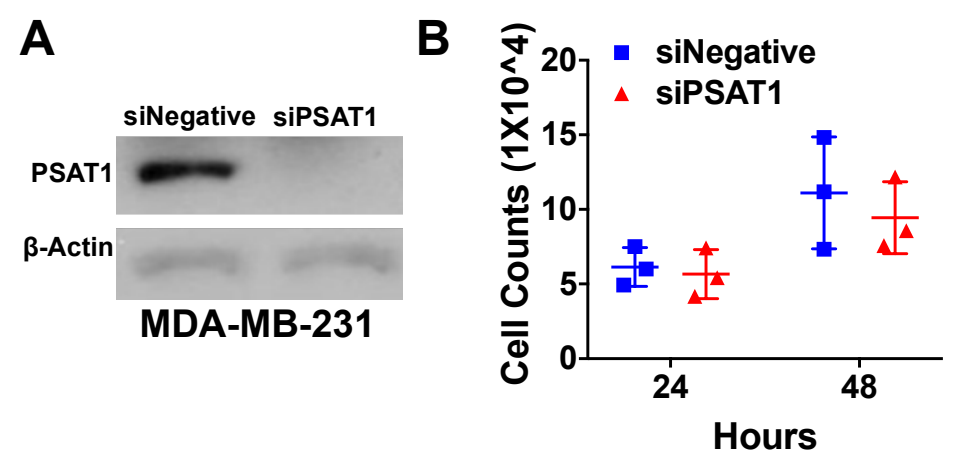

Figure 8: SiRNA suppression of PSAT1 has no proliferative effect. A) Representative western blot analysis demonstrating PSAT1 expression in MDAMB-231 transfected with control or PSAT1 specific siRNA. B) Cell proliferation at 24 and 48 hours after transfection with control or PSAT1 siRNA. Data is presented as viable cell counts and shown is mean \pm SD. Results were analyzed by 2-way ANOVA. 


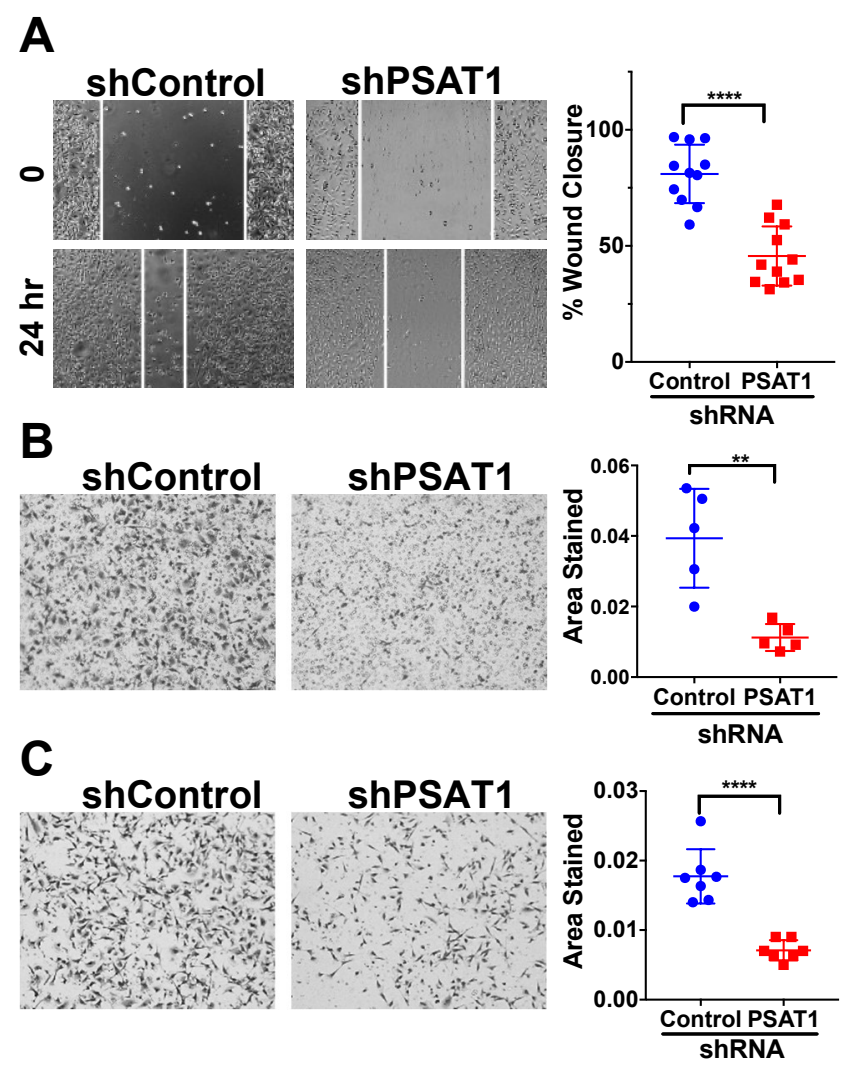

Figure 9: Loss of PSAT1 suppresses MDA-MB-231 motility and invasion in vitro. A) Representative images of wound healing assays for both control and PSAT1 silencing in MDA-MB-231 cell lines at 0 and 24 hours. Quantification is demonstrated as $\%$ wound closure (mean $\pm \mathrm{SD}$ ), $p<0.0001$ as determined by one-way ANOVA with Tukey's comparison test. B) Representative images for migration assays for both control and PSAT1 suppressed cells. Quantification is presented as area stained and shown are mean \pm SD, $p=0.0079$ as determined by one-way ANOVA with Tukey's comparison test. C) Representative images of Matrigel invasion assays with both stable shRNA MDA-MB-231 cell lines. Quantification is reported as area stained and represented as mean $\pm S D, p<0.0001$ as determined by unpaired $t$ test. Note: Controls are the same as those represented within the PHGDH experiments. 

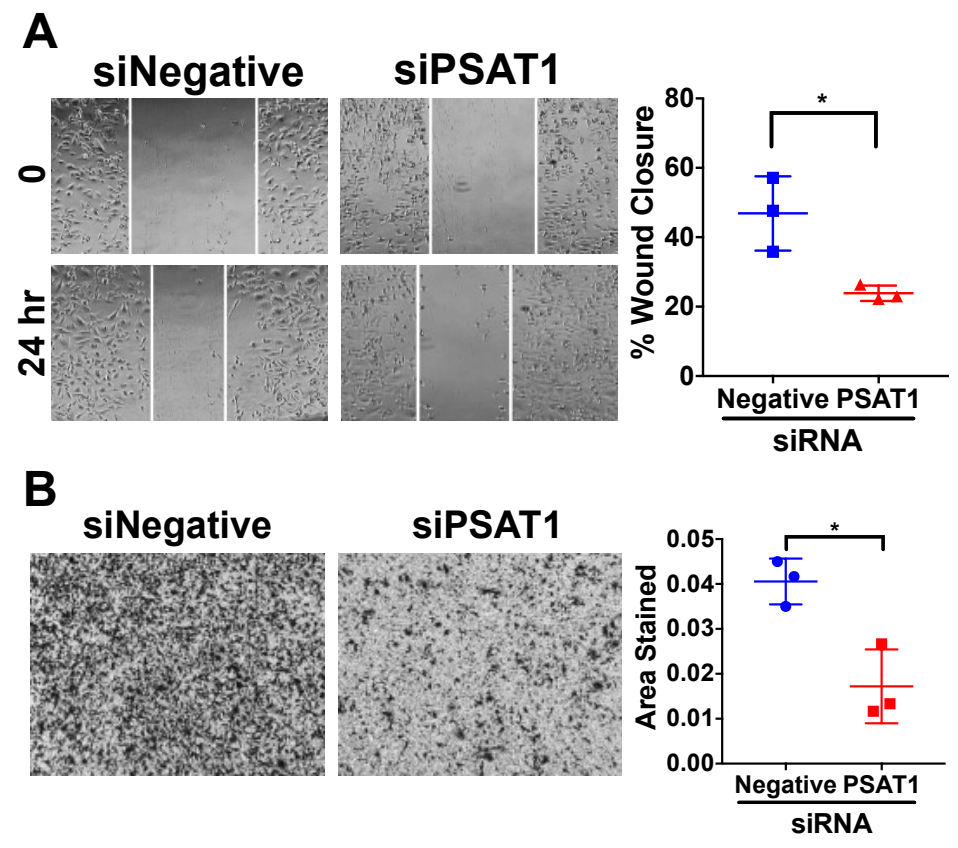

Figure 10: SiRNA suppression of PSAT1 decreases motility of MDA-MB231 cells in vitro. A) Representative images of wound healing assays of MDAMB-231 cells with transient suppression of PSAT1. Quantification is presented as $\%$ wound closure (mean $\pm \mathrm{SD}$ ), $\mathrm{p}=0.0217$ as determined by unpaired $t$ test. B) Boyden chamber migration assays were quantified as mean \pm SD area stained, $p=0.0139$ as determined by unpaired $t$ test. 
transwells and found that suppression of PSAT1 also significantly inhibited MDAMB-231 cell invasiveness (Figure 9C).

To determine if these results were unique to these cells or extend to other serine synthesis independent cell systems, we examined the effects of PSAT1 suppression on HCC1806 cells that exhibit comparable PSAT1 and PHGDH expression (Figure 6). Similar to the MDA-MB-231 cells, we observed no proliferative defect upon transient loss of PSAT1 (Figure 11A\&B). In addition, changes in HCC1806 actin stress fibers and cell morphology as seen with phalloidin staining was also comparable to the MDA-MB-231 cells upon PSAT1 suppression, wherein cells exhibited loss of elongated stress fibers (Figure 11C). Consistent with these findings, the migratory ability of these cells was also significantly inhibited in both the wound healing (Figure 12A) and Boyden chamber migration assays (Figure 12B) upon PSAT1 suppression. Taken together, these results suggest that while PSAT1 silencing does not adversely affect the proliferative capacity of serine synthesis-independent TNBC cells, PSAT1 loss does significantly inhibit TNBC motility and migration.

\section{Suppression of PHGDH does not phenocopy the loss of PSAT1 on migratory ability}

Given the differential levels of PHGDH between these cell lines, this data suggests that the function of PSAT1 in contributing to motility may be separate from its primary role in serine synthesis. To more directly examine this, we tested both MDA-MB-231 (low PHGDH) and HCC1806 (similar PHGDH expression to PSAT1) in the cell motility and migration models under both stable and transient 

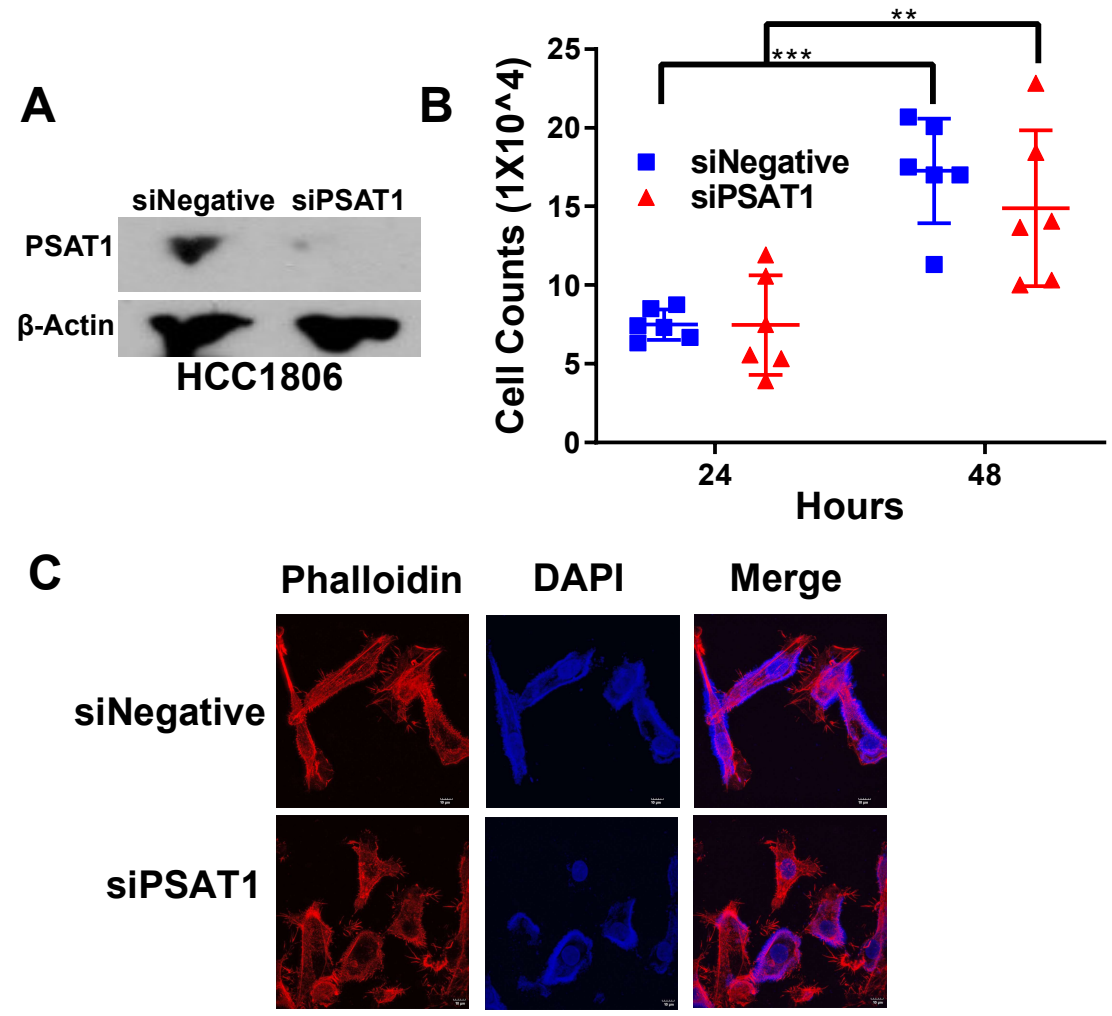

Figure 11: Suppression of PSAT1 in HCC1806 cells does not have an antiproliferative effect. A) Representative western blot of PSAT1 levels after transient transfection of negative control or PSAT1 siRNA. B) Cell proliferation measured via trypan blue exclusion at 24 and 48 hours. Data is shown as mean \pm SD of viable cell counts and analyzed by 2-way ANOVA, Control $p=0.0004$, PSAT1 $p=0.0061$. C) Representative images of cytoskeleton F-actin filaments upon phalloidin staining in both negative control and PSAT1 siRNA treated HCC1806 cells. 
A

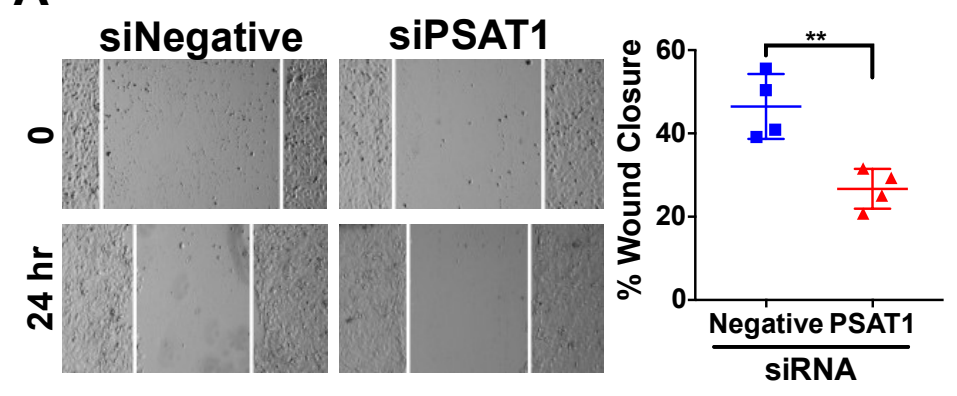

B
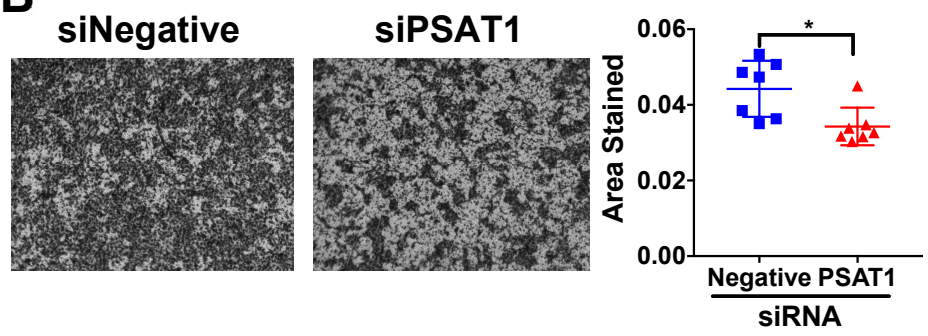

Figure 12: Decreased motility of HCC1806 cells upon suppression of PSAT1. A) Representative wound healing assay images. Quantification is presented as \% wound closure and shown is mean \pm SD, $p=0.005$ as determined by unpaired $t$ test. B) Image of cell migration after 24-hours. Quantification is presented as area stained and shown is mean $\pm S D, p=0.02$ as determined by unpaired $t$ test. 
suppression of PHGDH. As PHGDH catalyzes the rate-limiting step in cellular serine production, suppression of this enzyme will underscore the relevance of the SSP for the inhibitory effects we observe. Similar to previous studies, we found no significant effect on proliferation upon loss of PHGDH in the MDA-MB-231 cells (Figure 13A\&B and Figure 14A\&B). Unlike our studies with PSAT1 silencing, PHGDH suppression did not affect the cell morphology or the F-actin cytoskeletal structure as observed by phalloidin staining (Figure 14C). We also observed no significant inhibition in either the wound healing (Figure 15A and Figure 16A) or Boyden chamber migration assays (Figure 15B and Figure 16B), contrary to our PSAT1 studies.

Alternatively, we also investigated PHGDH function in the HCC1806 cells to demonstrate that these effects are not dependent simply on the dysregulation of the serine synthesis pathway. Similar to MDA-MB-231 cells, suppression of PHGDH did not affect HCC1806 proliferation (Figure 17A\&B), F-actin cytoskeletal arrangement compared to control cells (Figure 17C), nor inhibit the migratory ability of these cells as observed in wound healing (Figure 18A) or Boyden chamber migration (Figure 18B). These results demonstrate that suppression of PHGDH does not phenocopy the inhibitory effects on these metastatic characteristics that results upon PSAT1 silencing. This suggests that promotion of the metastatic characteristics seen in serine synthesis-independent TNBC is selective for PSAT1, which may be driven through a function unrelated to its role in de novo serine production.

\section{Suppression of PSAT1 inhibits experimental metastasis}



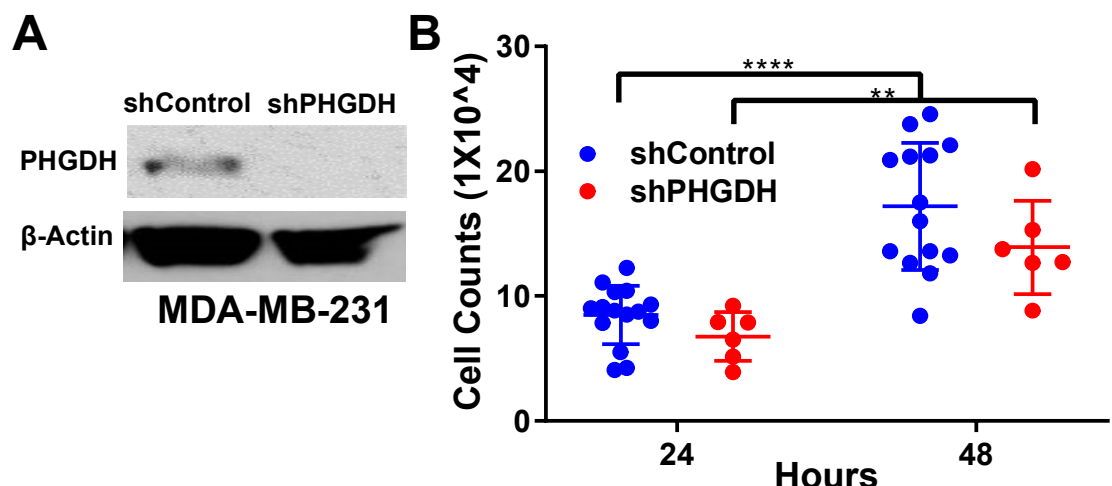

Figure 13: PHGDH is dispensable for MDA-MB-231 proliferation in vitro. A)

Representative western blot analysis demonstrating PHGDH expression in MDA-MB-231 cells stably expressing control or PHGDH specific shRNA. B) Cell proliferation at 24 and 48 hours with or without PHGDH expression. Data is presented as viable cell counts and shown are mean \pm SD for both control and PHGDH shRNA. Results were analyzed by 2-way ANOVA, Control $p<0.0001$, PHGDH $p=0.0057$. Note: Controls are the same as those represented within the PSAT1 experiments. 


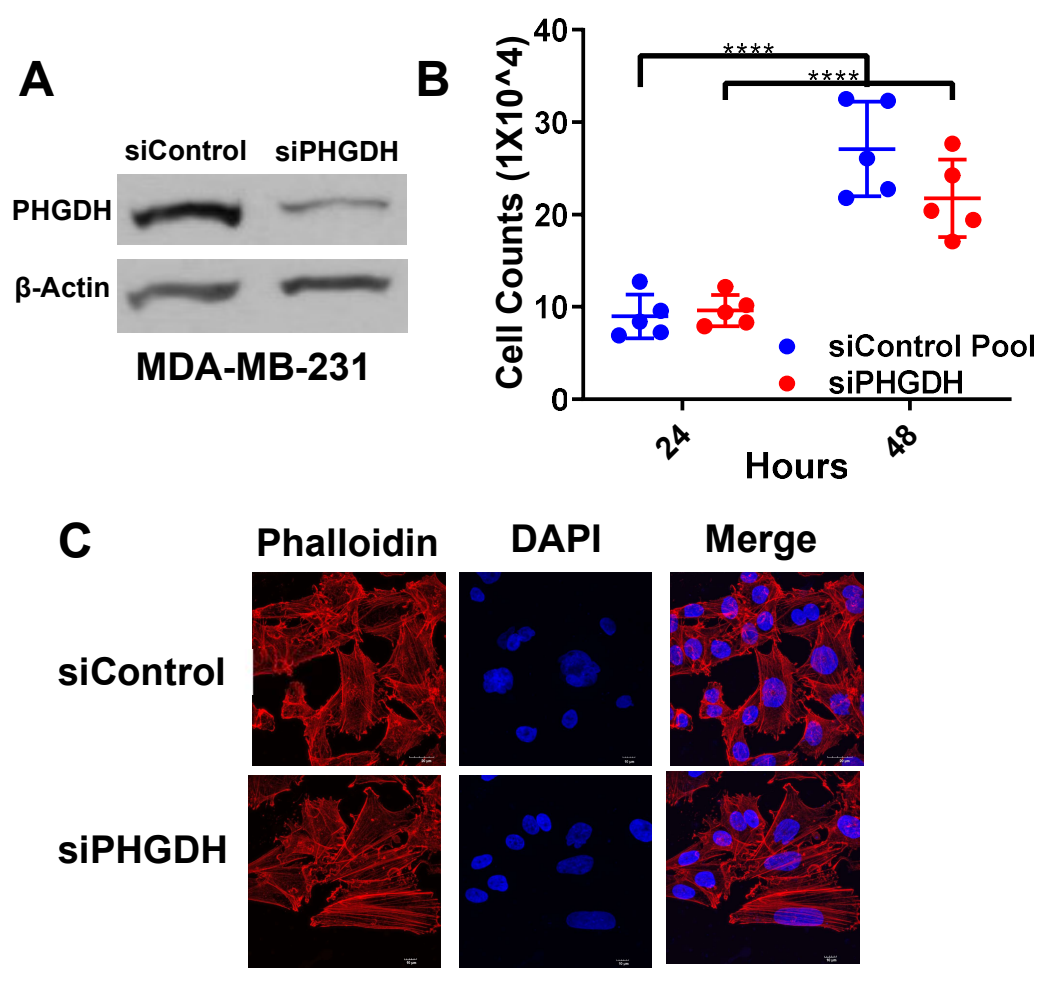

Figure 14: SiRNA suppression of PHGDH in MDA-MB-231 cells has no effect on in vitro proliferation. A) Representative western blot analysis demonstrating PSAT1 expression in MDA-MB-231 transfected with control of PHGDH specific siRNA. B) Cell proliferation at 24 and 48 hours after transfection with control or PHGDH siRNA. Data is presented as viable cell counts and shown is mean $\pm S D$. Results were analyzed by 2-way ANOVA, $p<0.0001$. C) Representative images of F-actin filaments after phalloidin staining in control or PHGDH siRNA treated MDA-MB-231 cells. 

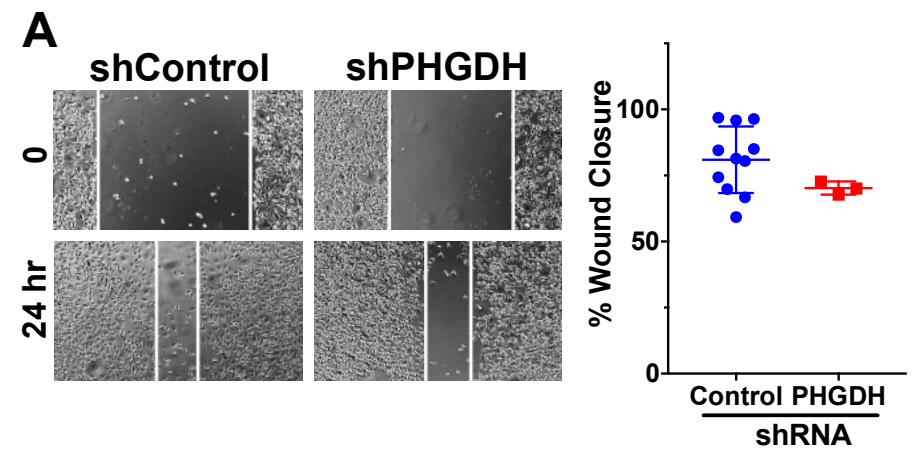

B

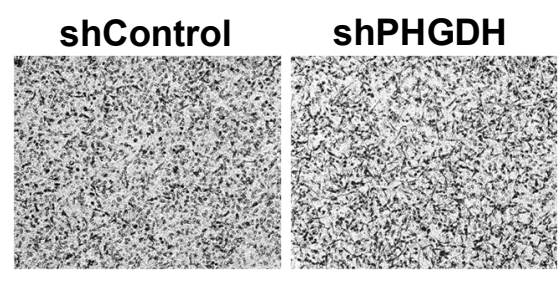

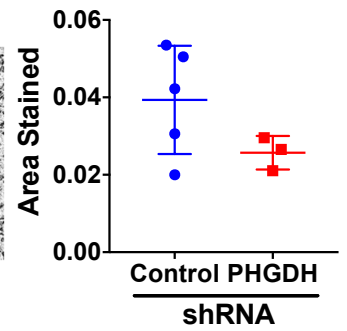

Figure 15: PHGDH is dispensable for MDA-MB-231 motility in vitro. A) Wound healing assays twenty-four hours post-scratch. Data is demonstrated as $\%$ wound closure and shown are mean $\pm S D, p=0.9810$ as determined by one-way ANOVA. B) Representative images for Boyden chamber migration assays. Quantification is presented as area stained (mean \pm SD), $p=0.6477$ as determined by one-way ANOVA. Note: Controls are the same as those represented within the PSAT1 experiments. 


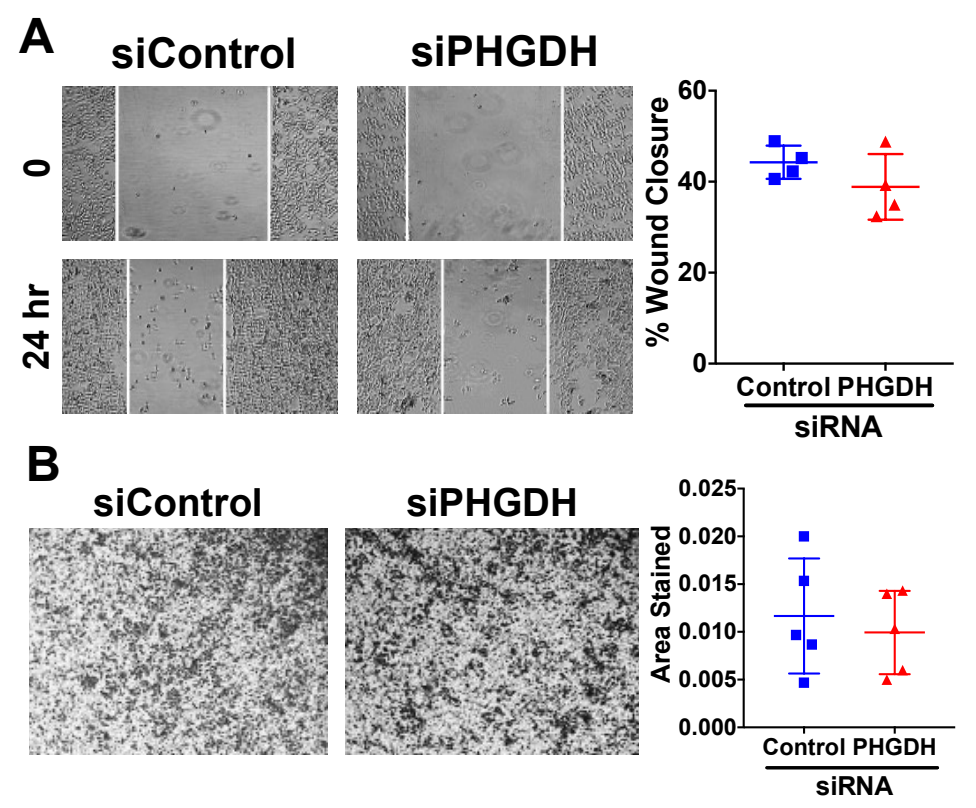

Figure 16: SiRNA suppression of PHGDH in MDA-MB-231 cells has no effect on motility or migration. A) Representative images of wound healing assays of MDA-MB-231 cells with transient suppression of PHGDH. Quantification is presented as $\%$ wound closure (mean \pm SD), $p=0.2284$ as determined by unpaired $t$ test. B) Boyden chamber migration assays were quantified as mean $\pm S D$ area stained, $p=0.6160$ as determined by unpaired $t$ test. 

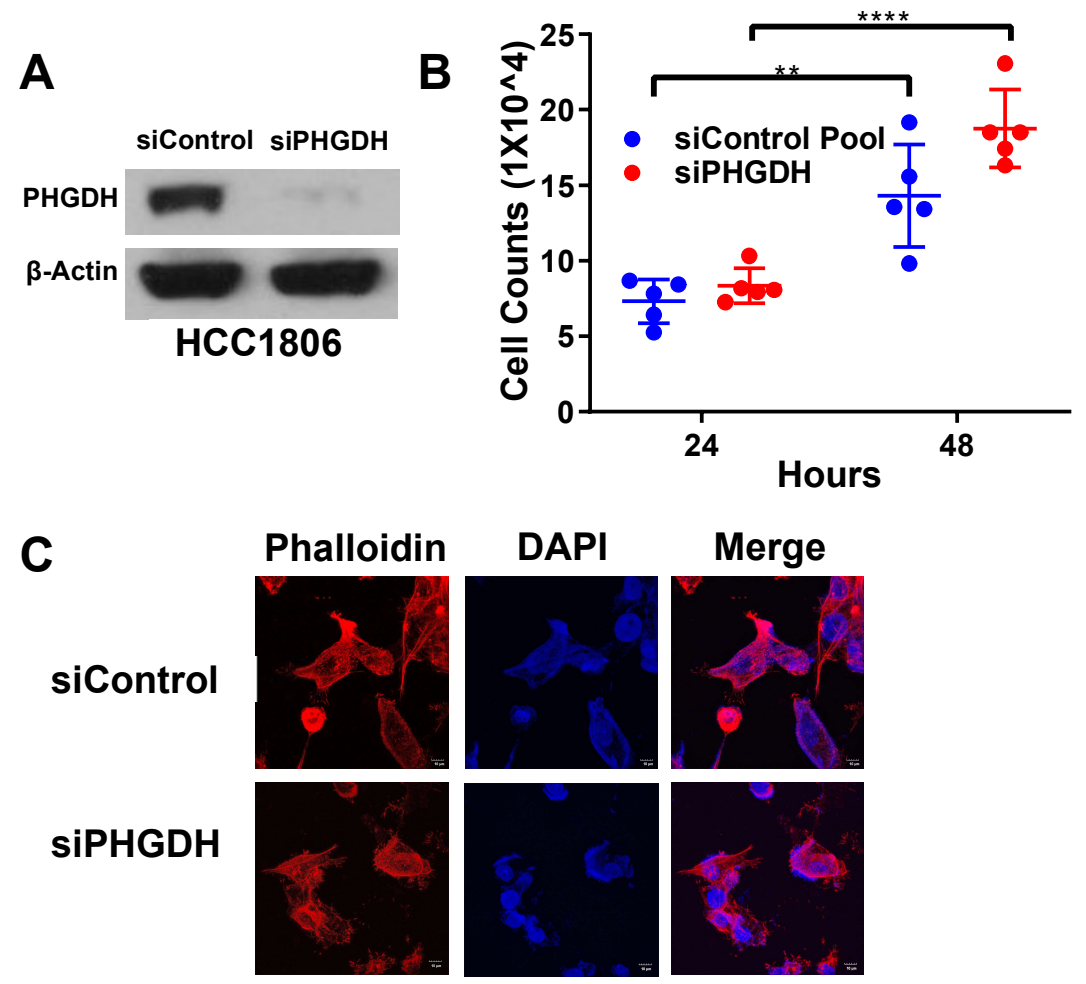

Figure 17: Loss of PHGDH does not affect HCC1806 proliferation. A) Western blot analysis for PHGDH expression in HCC1806 cells after transient transfection of control or PHGDH siRNA. B) Cell proliferation at 24 or 48 hours, which is presented as viable cell counts (mean \pm SD). Results were analyzed by 2-way ANOVA, Control $p=0.0011$, PHGDH $p<0.0001$. C) Representative images of phalloidin stained F-actin filaments upon PHGDH suppression in HCC1806 cells. 

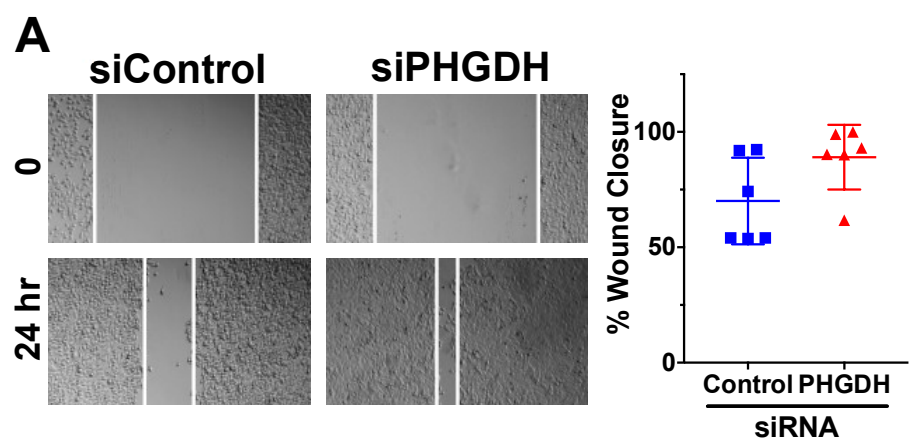

B

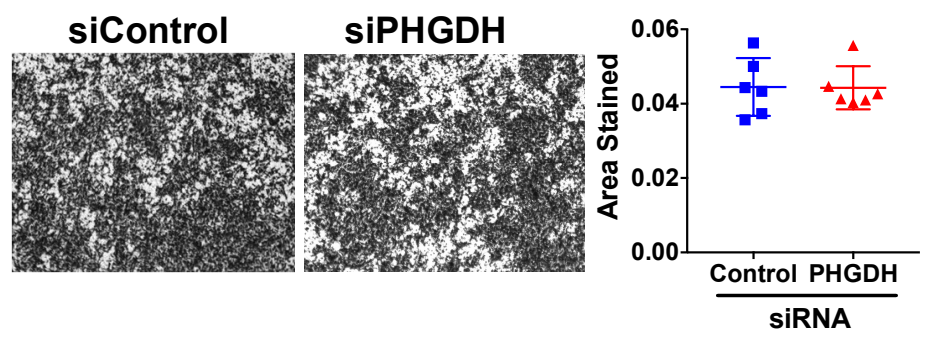

Figure 18: Loss of PHGDH does not suppress HCC1806 motility or migration potential. A) Representative images and quantification of wound healing in HCC1806 cells. Data is presented as \% wound closure and shown are mean $\pm S D, p=0.0754$ as determined by unpaired $t$ test. B) Boyden chamber migration assays of HCC1806 cells with or without PHGDH. Quantification is demonstrated as area stained (mean $\pm \mathrm{SD}$ ), $p=0.9563$ as determined by unpaired t test. 
To further investigate the potential role of PSAT1 in TNBC metastasis, we utilized an established mouse model of experimental metastasis entailing TNBC lung nodule formation after tail-vein injection (Figure 19A) [99]. Sixty days postinjection, we found a significant decrease in the number metastatic foci from MDAMB-231 cells lacking PSAT1 compared to mice injected with control cells (Figure 19B\&C). We also found that the no injection mice did not develop tumor nodules during the course of this experiment (Figure 19B). To ensure that the decrease observed was not due to a loss of anchorage-independent growth, we examined both control and PSAT1 silenced cells for the ability to grow in soft agar. We found that loss of PSAT1 does not affect anchorage-independent growth of these cells (Figure 20), supporting that loss of PSAT1 inhibits the metastatic potential of TNBC in vivo. 


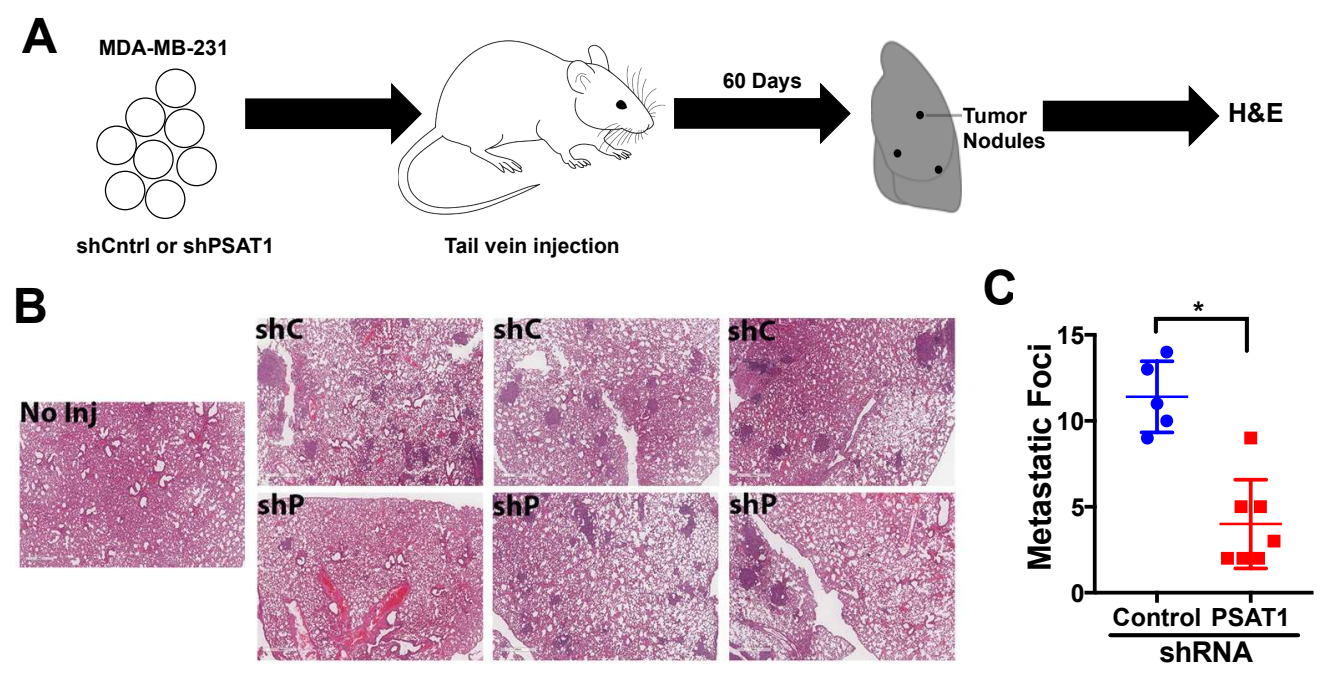

Figure 19: PSAT1 silencing inhibits MDA-MB-231 experimental metastasis. A) Outline of experimental protocol for the in vivo study. B) Representative H\&E staining (4X) of lung sections from individual mice receiving no injection or MDA-MB-231 cells with or without stable knock-down of PSAT1. Micro-metastases are indicated by the dark punctate areas of staining. C) Micro-metastases were quantified as the sum across three image fields under $4 \mathrm{X}$ magnification from an individual mouse lung section $(\mathrm{N}=5$, shControl; $\mathrm{N}=7$, shPSAT1) and presented as metastatic foci (mean $\pm \mathrm{SD})$. No micro-metastases were found in no injection control animals. Data analyzed by non-parametric unpaired t-test with Mann-Whitney $U$ test, ${ }^{*} p=0.0051$. 
A

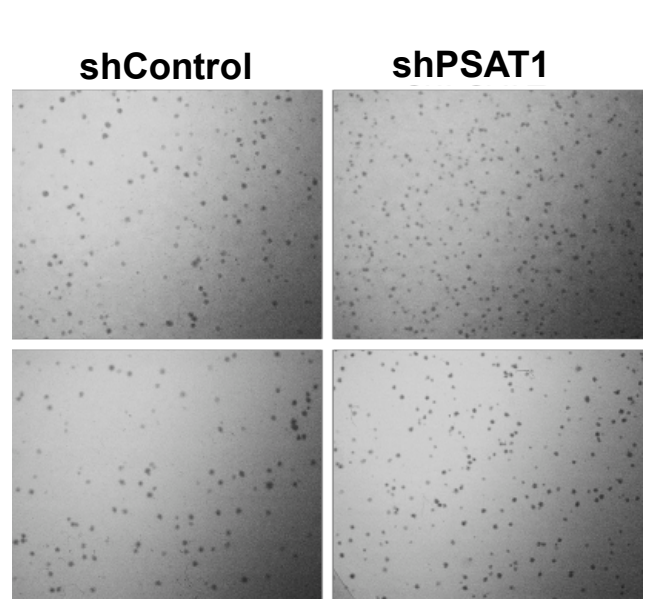

MDA-MB-231

B

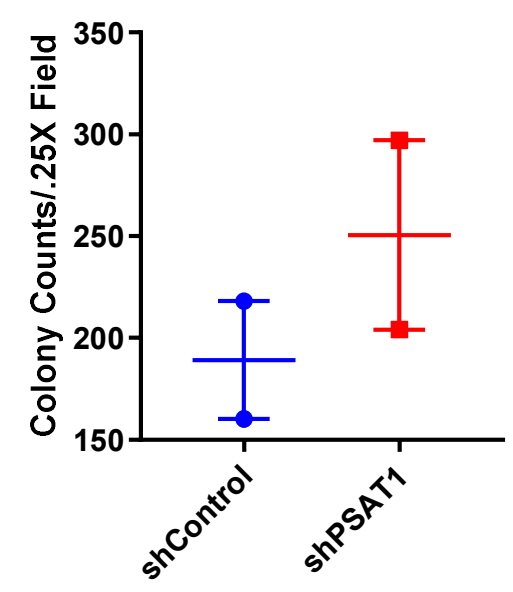

Figure 20: Suppression of PSAT1 does not affect anchorageindependent growth of MDA-MD-231 cells. A) Soft agar colony formation assays of MDA-MB-231 cells stably expressing control or PSAT1-specific shRNA. Shown are representative images of colonies two weeks after plating from two independent experiments. B) Quantification of soft agar assays. Data points are individual experiments. Statistical analysis not performed as $n=2$. 


\section{Discussion}

In this report, we demonstrate that PSAT1 expression increases in higher grades of TNBC. Unlike PHGDH, no genetic amplifications have been observed for PSAT1 and elevated levels are the result of oncogenic pathways that are activated as TNBC progresses. Multiple studies have previously demonstrated that activating transcription factor 4 (ATF4) can control PSAT1 expression. ATF4 has been shown to regulate PSAT1 in several different cancer types, including breast cancer [46-49]. Importantly, in all tumors examined, ATF4 activation increases expression of not only PSAT1 but also enzymes within the entire serine synthesis pathway, including PHGDH and PSPH. As this is not selective for PSAT1 expression, as we have found in the MDA-MB-231 cells, this suggests other regulatory mechanism(s) in TNBC that may be directly controlling PSAT1.

Within breast cancer, the transcription factors TAZ/YAP have been shown to have higher activity in basal breast cancer subtype and to selectively induce expression of PSAT1 in TNBC, specifically in the MDA-MB-231 cell line [60]. TAZ expression is activated by the overexpression of the transcription factors Twist and Snail [61]. This increased TAZ/YAP expression/activity has been correlated with high histological grade breast cancer [61] as well as the promotion of oncogenic transformation, enhancing tumorigenic properties, induction of cancer stem celllike activity and resistance to breast cancer drug therapies [100]. TAZ/YAP have also been linked to increased metastatic capability in breast cancer [101]. In addition, PSAT1 has also been shown to be induced by mTOR and be under the regulation of the PI3K/Akt/mTOR pathway in breast cancer [62]. This pathway has 
been demonstrated to increase as breast cancer progresses with the highest expression levels correlating with higher grade mammary tumors [102]. The $\mathrm{PI} 3 \mathrm{~K} / \mathrm{Akt} / \mathrm{mTOR}$ pathway has also been highly associated with metastasis [102]. Taken together, multiple pro-tumorigenic pathways that mediate breast cancer progression converge to increase PSAT1 expression that then may contribute to the metastatic potential of TNBC.

We now show that suppression of PSAT1 in two defined serine synthesisindependent TNBC cell lines does not affect cell proliferation, yet, there is a significant reduction in the in vitro metastatic capabilities of cell migration and invasion due, in part, to alterations in F-actin cytoskeletal rearrangement and cell morphology. Despite substantial PHGDH expression in a representative cell type (HCC1806), this anti-metastatic effect seems to be selective for PSAT1 as PHGDH suppression did not phenocopy loss of PSAT1. Further, PSAT1 contribution to metastatic activity extended in vivo as protein suppression significantly inhibits tumor nodule formation without disrupting the anchorage-independent growth of these cells. As the tail-vein injection experimental model is limited in its assessment of the entire metastatic cascade, particularly tumor cell escape from a primary tumor, future examination of effects of PSAT1 loss in metastatic potential from primary orthotopic tumors will be necessary to fully assess its function in TNBC metastasis. Yet, these results suggest that PSAT1 may have an alternate function in contributing to TNBC metastasis that is separate from its role in serine synthesis.

It is important to note that these studies were carried out in TNBC cell types 
that have been designated as independent of de novo serine synthesis. While expressing SSP enzymes, there is little to no metabolic flux through the SSP in these cells, thereby necessitating a requirement for external serine for cell proliferation. Conversely, it is well documented that there are TNBC cell lines and patient tumors that are designated as serine synthesis-dependent. This subset exhibits extremely high expression of PHGDH primarily due to genetic amplifications. This drives increased flux through the SSP and allows for growth in serine lacking conditions. Yet, even in serine complete conditions, suppression of PHGDH or PSAT1 causes a significant reduction in cell proliferation in vitro due to disruptions in glutaminolysis and nucleotide production [49,87]. In addition, suppression of PHGDH, or the use of PHGDH inhibitors, have been shown to have a detrimental effect on the growth of these tumors in vivo $[86,87]$.

While serine synthesis-dependent tumor cells are sensitive to PHGDH inhibitors, these compounds had no effect on serine synthesis-independent cell types [86] and furthermore did not affect serine flux within this subset [87]. Consistent with these results, suppression of PSAT1 or PHGDH also did not affect the proliferative capacity in the MDA-MB-231 cells in our studies $[55,87]$. Conversely, others have also demonstrated that overexpression of PSAT1 in other independent cell types was able to increase proliferation and metastatic capability [49]. Whether these effects were directly due to increased de novo serine synthesis is unclear but supports a role for PSAT1 in promoting cell metastasis. These previous findings coupled with our results demonstrating the metastatic inhibitory 
effects upon suppression of PSAT1 indicate a selective role for PSAT1 and not a result of dysregulation of de novo serine production.

While these results suggest an activity for PSAT1 apart from serine synthesis, its pro-metastatic function(s) in these cell types is unclear. There have been several mechanisms proposed for non-canonical functions of PSAT1. For example, in esophageal cancer, PSAT1 acts upstream of Akt that controls downstream targets of GSK-3B and snail, which work to promote tumor progression and enhance metastatic characteristics [72]. In non-small cell lung cancer, it has been suggested that PSAT1 inhibits the degradation of cyclin D1, which leads to an alteration in the Rb-E2F pathway [81]. However, despite these potential non-canonical activities of PSAT1, decreased PSAT1 generally resulted in loss of cell proliferation. This is not evident in our systems and suggests another yet unknown function towards metastatic potential. Alternatively, Yang et al postulates that TAZ/YAP's regulation of PSAT1 is driven by a need to generate $\alpha$ ketoglutarate in order to feed into the TCA cycle. They found that the serineindependent MDA-MB-231 cell line exhibits a high level of TAZ/YAP and are designated as glutamine dependent [60]. This observation from Yang et al combined with the implications of TAZ/YAP in metastasis indicates that this might be a mechanism in which PSAT1 is contributing to the metastatic characteristics of TNBC. In addition, the observed disruption in actin fibers upon suppression of PSAT1 indicates that PSAT1 may contribute particularly to metastatic potential by maintenance of cytoskeleton structure in promoting cell motility. 
Given the extent of PSAT1 expression across TNBC, even within subsets not dependent on de novo serine synthesis, suggests that it may serve an alternate function. These studies support a non-canonical role as loss of PHGDH did not phenocopy PSAT1 suppression with regards to metastatic potential. Continued work will be necessary to define whether PSAT1 functions in a similar manner in additional TNBC cell types and the mechanisms by which PSAT1 supports TNBC metastasis. Yet, despite the uncertainty of these mechanism, we believe that PSAT1 is an appealing potential therapeutic target for TNBC patients; particularly in suppressing metastatic spread. 


\section{Extended Results and Discussion}

In addition to the PHGDH suppression experiments that were done, we also performed metastatic characteristics assays in the presence of exogenous serine (Figure 21). Although the culture media for MDA-MB-231 cells contain 400uM serine, we wanted to ensure that serine did not become limiting in our studies and that our results are truly independent of a requirement for de novo serine synthesis and utilization. We observed that the addition of exogenous serine $(2.5 \mathrm{mM})$ did not rescue the PSAT1 suppression phenotype in either our wound healing (Figure 21) or our migration assays (Figure 22). Since addition of exogenous serine was unable to rescue this phenotype these data further suggests that PSAT1 contributes to TNBC metastasis independent of its role in serine synthesis.

Thus, to address the non-canonical function of PSAT1 that we observed in these studies, we have preliminarily attempted to identify potential binding partners of PSAT1 that could contribute to its anti-metastatic function. Database analysis revealed several potential binding partners of PSAT1 that were identified through large high throughput interaction screens. Two proteins that may influence this metastatic phenotype are of particular interest. Tissue inhibitor of metalloproteinases 2 (TIMP2), when expressed and active, typically inhibits tumor growth, invasion, and metastasis via its inhibition of the matrix metalloproteinases (MMPs) [103]. However, there have been reports of TIMP2 having multiple roles and that in breast cancer there is evidence to support TIMP2's involvement in promoting proliferation, protecting against apoptosis, and correlating to poorer progression-free survival [103] The contradictory roles of TIMP2 as both a 

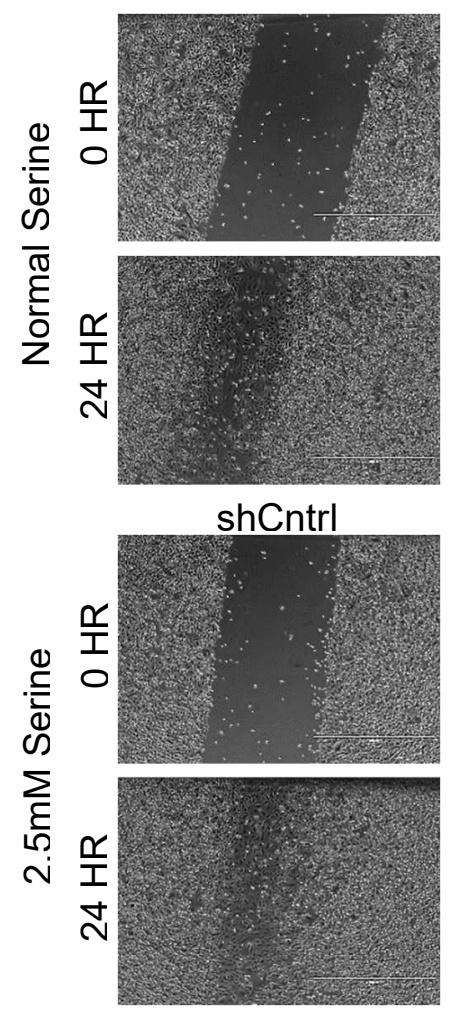

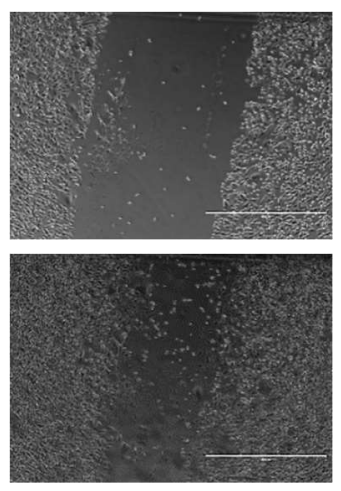

ShPSAT1

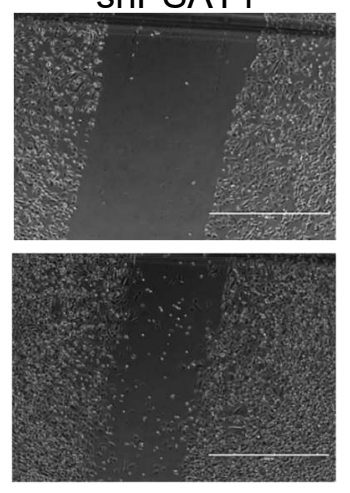

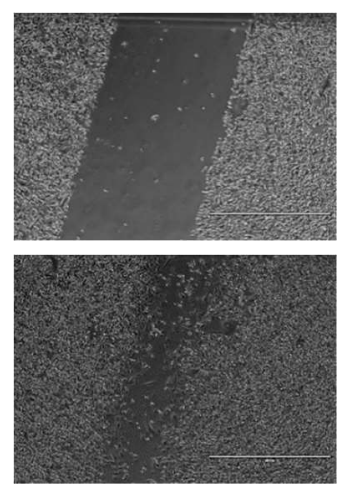

shPHGDH

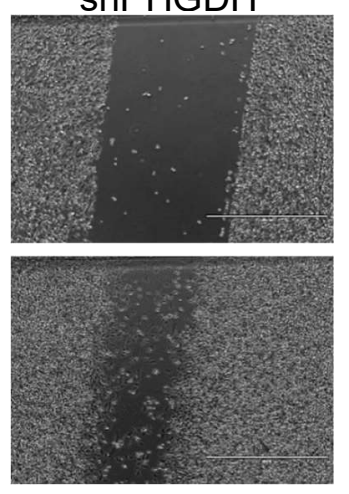

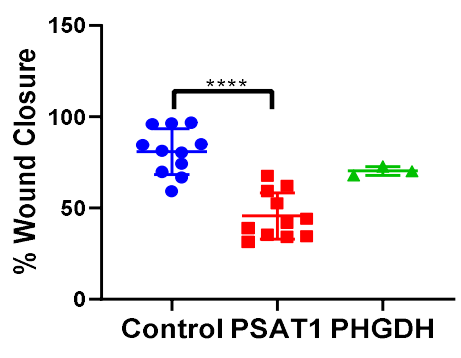

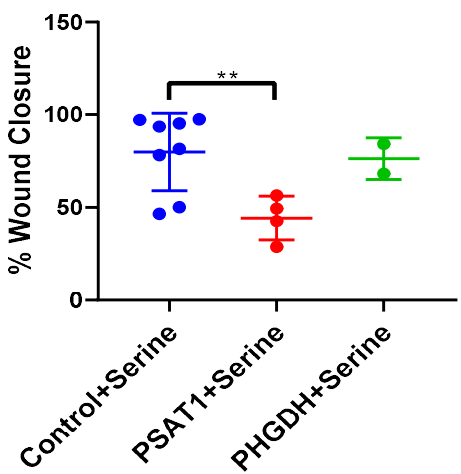

Figure 21: Exogenous serine does not rescue PSAT1 suppression on cell motility. Representative images of wound healing at 24 hours post scratch with or without the addition of $2.5 \mathrm{mM}$ exogenous serine for the MDA-MB-231 cells transfected with shRNA against control, PSAT1, or PHGDH. Data analyzed by one-way ANOVA, ${ }^{* * * *} p<0.0001,{ }^{* *} p=0.0035$. 


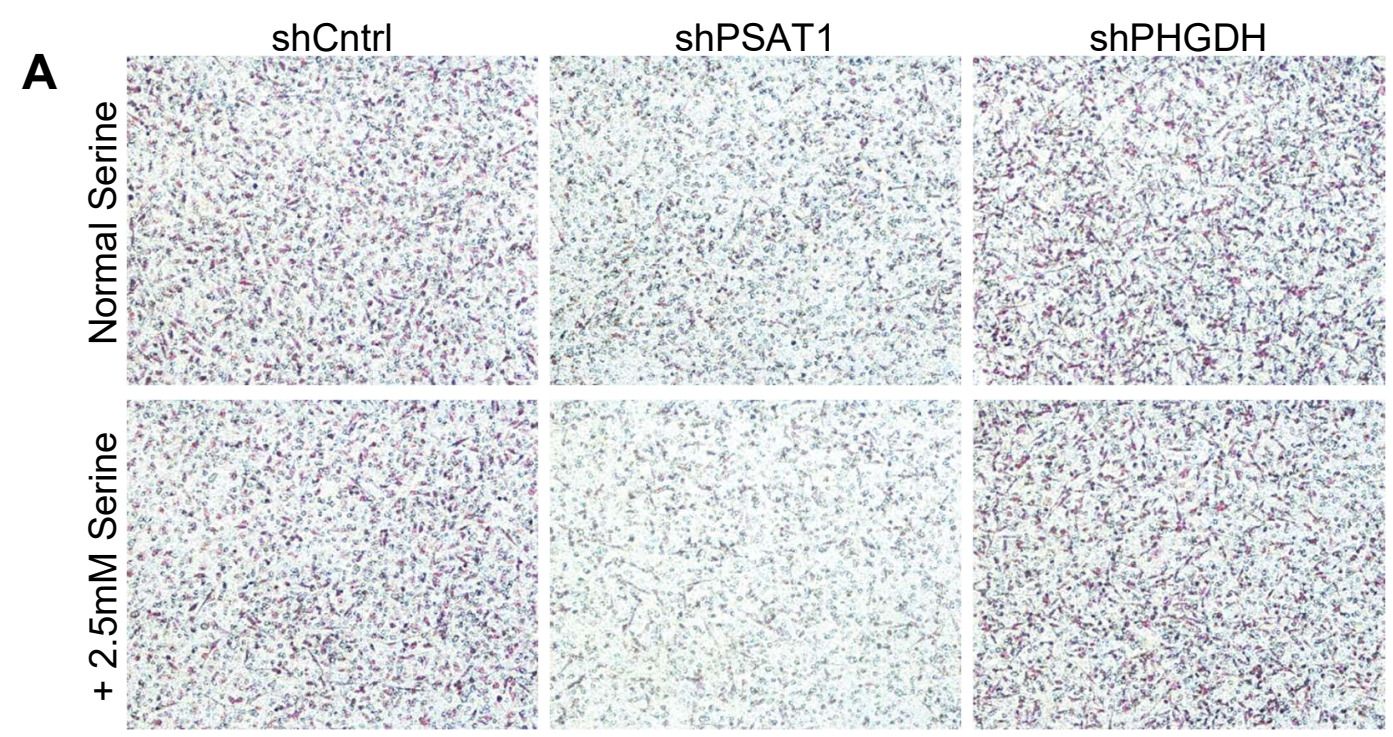

B
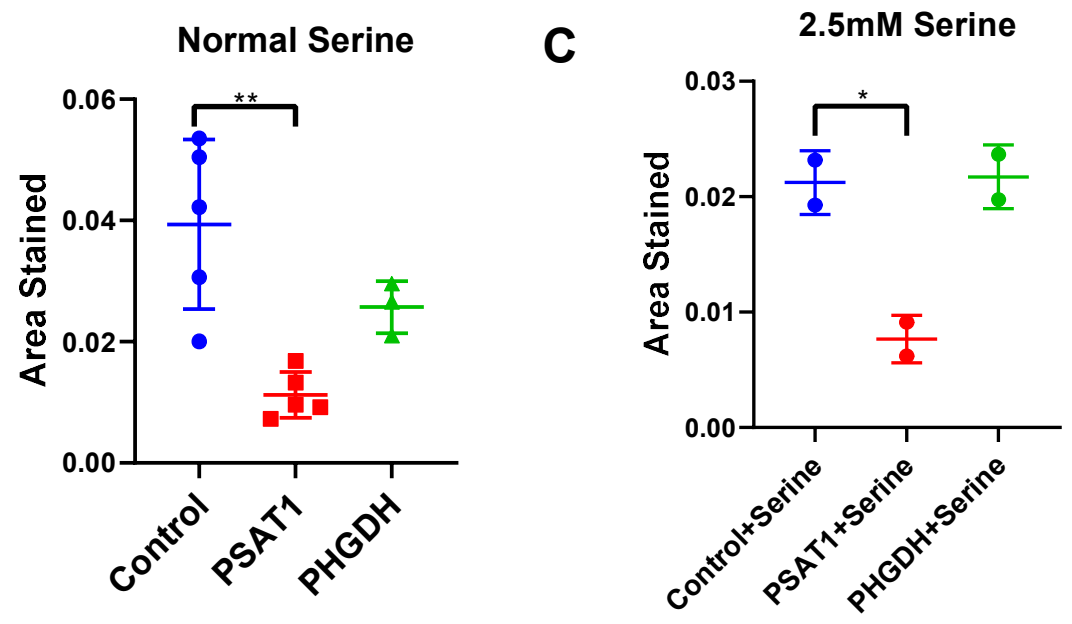

Figure 22: Exogenous serine does not rescue effect of PSAT1 suppression on cell migration. A) Representative images of Boyden chamber migration assays 24 hours post seeding with or without the addition of exogenous serine for the MDAMB-231 cells transfected with shRNA against control, PSAT1, or PHGDH. B) Quantification of migration assays under normal serine conditions. Data analyzed by one-way ANOVA, $p=0.0021$. C) Quantification of migration assays with $2.5 \mathrm{mM}$ Serine. Data analyzed by one-way ANOVA, $p=0.0264$. 
correlator with low recurrence and poorer prognosis [104] could possibly be due to its interacting partners. To confirm an interaction between PSAT1 and TIMP2, I performed a co-immunoprecipitation assay (Figure 23) and found an association between PSAT1 and TIMP2. It is plausible that the interaction of TIMP2 and PSAT1 interferes with the ability of TIMP2 to inhibit MMPs and thus may promote a pro-metastatic phenotype. However, upon loss of PSAT1, the TIMP2-PSAT1 interaction is abolished and TIMP2 is able to inhibit the MMPs and thus result in the inhibition of the metastatic characteristics. Further studies to evaluate this mechanism include endogenous co-immunoprecipitation studies to confirm the interaction between PSAT1 and TIMP2. In addition, MMP activity assays would also be performed utilizing both control and PSAT1 suppressed cells in order to determine if PSAT1 suppression, and potentially loss of PSAT1-TIMP2 interaction, has an effect on MMP activity.

A second potential interacting protein suggested was ras responsive element binding protein 1 (RREB1). This protein's drosophila homolog, hindsight (HNT), has been shown to be involved in collective cell migration, the regulation of cell adhesion, and to play a part in cell morphology [105]. These affects were shown to occur via the JNK and Stat pathways. Additionally, it was shown in breast epithelial cells that the expression of RREB1 was required for cell spreading and migration as suppression of RREB1 did not affect cell viability but rendered the cells immobile [105]. Thus, in an alternative mechanism, the dramatic differences that we observed on cell morphology and migration upon PSAT1 suppression could be attributed to a loss of the interaction between PSAT1 and RREB1. Either 


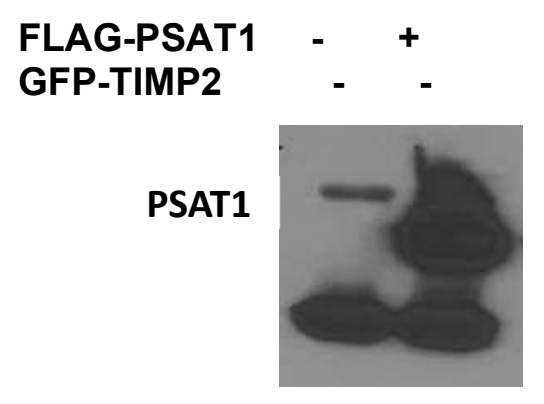

IP: FLAG

IB: PSAT1

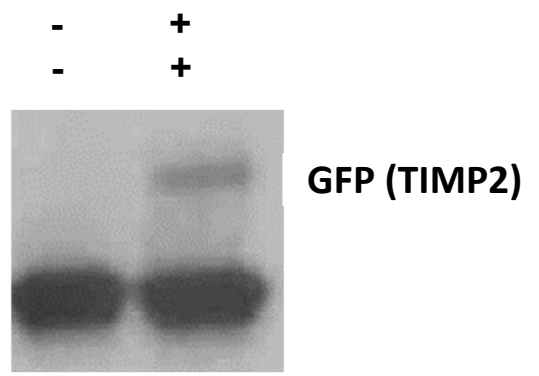

IP: FLAG

IB: GFP

Figure 23: PSAT1 and TIMP2 are binding partners. Western blot analysis following immunoprecipitation of FLAG-PSAT1. Lysates were immunoprecipitated using M2-FLAG beads and membranes were immunoblotted for either PSAT1 or GFP, as a measure of TIMP2 expression. 
of these mechanisms may be valid means by which PSAT1 is contributing to the metastatic characteristics of TNBC independent of its metabolic activity. Further work would need to be done to fully investigate PSAT1's role as it may relate to these pathways. 


\section{CHAPTER 3}

\section{PHOSPHOSERINE AMINOTRANSFERASE 1 (PSAT1) AND THE SERINE SYNTHESIS PATHWAY ALTERS SENSITIVITY TO 4-HYDROXYTAMOXIFEN \\ TREATMENT IN ESTROGEN-RECEPTOR POSITIVE BREAST CANCER}

\section{Introduction}

Breast cancer is the most common cancer that afflicts US women [3]. Importantly, breast cancer is not a singular disease, but is characterized by several different histological subtypes that have now been identified. Estrogen receptor positive breast cancer $(E R+B C)$ is the most common breast cancer subtype [3] and it accounts for $65 \%$ to $75 \%$ of all breast cancer cases [3]. ER+BC has a higher incidence rate in older white women living in more western countries, like the United States [7]. ER+ tumors express the estrogen receptor and the growth/proliferation of these tumors are driven by estrogens binding to the estrogen receptor and promoting transcription of pro-proliferative genes [10]. As these tumors are reliant upon this estrogen-driven growth, therapies that target the estrogen signaling pathway have become successful treatment options for patients and much of the decline in mortality associated with breast cancer is credited to their success [3].

All endocrine therapies target portions of estrogen signaling through different mechanisms. The first clinical endocrine therapy, Tamoxifen, was originally classified as an anti-estrogen because it functions as an estrogen 
antagonist in the breast, however, in other tissues it has agonistic properties [10]. It has thus been reclassified as a selective estrogen receptor modulator (SERM). Tamoxifen can function on both estrogen receptor $\alpha(E R \alpha)$ and estrogen receptor $\beta$ (ER $\beta$ ) [106]. When tamoxifen is bound to ER $\beta$ it acts as a pure antiestrogen, however, when tamoxifen is bound to ER $\alpha$ it can act as a partial agonist [106]. Tamoxifen's primary mechanism of action in the breast is through competition with estrogens binding to the estrogen receptor that results in the inhibition of the estrogen driven pro-proliferative transcription program [12,13]. In addition to binding to the estrogen receptor, tamoxifen has also been demonstrated to recruit corepressors to the estrogen response elements in the promoters of estrogen receptor target proteins as an additional mechanism of inhibition [106].

Fulvestrant, $\mathrm{ICl}$, is considered a pure antiestrogen that exhibits no agonistic activity $[10,107]$. Fulvestrant is also classified as a selective estrogen receptor degrader (SERD) [9]. Fulvestrant differs from tamoxifen in its mechanism of action as fulvestrant binds to the estrogen receptor and increases protein degradation resulting in attenuation of the estrogen driven pro-proliferative transcriptional program [12]. Fulvestrant also has a higher binding affinity for ER compared to tamoxifen and is a more potent inhibitor of cell proliferation [107]. Fulvestrant is often given to patients that have had disease progression following tamoxifen treatment [10]. Fulvestrant can be used as a second line treatment because it has been shown to have no cross-resistance with tamoxifen [107].

Despite numerous effective therapeutic options for patients with $E R+B C$ and a better patient prognosis, there are more deaths linked to $E R+B C$ than any 
other subtype [11]. This is largely attributed to resistance to endocrine therapy, which can be classified as either innate or acquired resistance. Innate, or de novo, resistance relates to patients that have no initial response to endocrine therapy [11]. Acquired resistance includes patients that initially respond to endocrine therapy but then experience disease recurrence or progression [11]. Clinically, the issue of endocrine resistance affects between $10 \%$ and $15 \%$ of all patients within five years of diagnosis [22]. By 15 years following diagnosis, it is estimated that $30 \%$ of ER+BC patients will become resistant to endocrine therapies [22].

With the potential for endocrine resistance to affect $30 \%$ of all ER+BC patients, there is an increased need to identify genes that may promote loss of response to these therapeutic options. One of the genes that has been linked to endocrine resistance is phosphoserine aminotransferase 1 (PSAT1) [82]. PSAT1, along with phosphoglycerate dehydrogenase (PHGDH) and phosphoserine phosphatase (PSPH) comprise the serine synthetic pathway. PSAT1 has been implicated in numerous different cancer types as contributing to proliferation, resistance, metastasis and has been implicated in breast cancer resistance to endocrine therapy $[49,57,58,65,72,81,82,97]$. In these initial reports concerning PSAT1 in breast cancer, it was determined that the expression of the PSAT1 transcript correlated to poorer progression-free survival in ER+ patients that were treated with tamoxifen with the PSAT1 correlation being the most significant [82]. This was later confirmed at the protein level [97] and this study also suggested an increase in PHGDH expression in tamoxifen insensitive breast cancer [97]. 
Together, these results suggest a potential metabolic requirement for SSP acting in promoting endocrine resistance.

In this report, we examine the clinical relevance of PSAT1 and PHGDH in tamoxifen-resistant $\mathrm{ER}+\mathrm{BC}$ and using in vitro models seek to determine if sensitivity to tamoxifen treatments or antiestrogens can be altered by manipulation of PSAT1 expression. 


\section{Methods and Materials}

\section{Chemicals}

(Z)-4-Hydroxytamoxifen was obtained from Sigma (H7904) and was dissolved in 100\% pure Ethyl Alcohol (Sigma - E7023). ICI 182,780 (Fulvestrant) was obtained from TocrisBioscience (CAS No: 129453-61-8) and was dissolved in dimethyl-sulfoxine (Fisher BioReagents - BP231). Concentrated stock solutions were prepared, aliquoted and stored at $-20^{\circ} \mathrm{C}$ for a period of 30 days. Diluted working stocks were prepared fresh for each application.

\section{Cell Culture}

MCF-7 cells were purchased from ATCC. LCC9 and LY2 cells were provided by Dr. Carolyn Klinge under an approved material transfer agreement from Dr. Robert Clarke $[108,109]$. All cells were cultured in IMEM supplemented with $5 \% \mathrm{FBS}$ and gentamicin. All cells were incubated at $37^{\circ}$ in $5 \% \mathrm{CO}_{2}$.

\section{Plasmid Construction and Transfections}

Human PSAT1 was generated from cDNA prepared from the LCC9 cell line. RNA was extracted using the RNeasy kit (Promega) and $1 \mathrm{mg}$ of RNA was used to produce cDNA using the reverse transcriptase kit (Thermo) with random primers. Primers homologous to the 5' and 3' ends of the mature RNA encoding for PSAT1 were adapted to include restriction sites for ECOR1 (5') and BAMH1 (3'). Polymerase chain reactions were performed to amplify PSAT1 from LCC9 cDNA preparation. PCR products were separated via gel electrophoresis and bands corresponding to the correct PCR product were excised from the gel and purified. PSAT1 fragments underwent restriction digest with ECOR1 and BAMH1 and then 
were ligated into the pcDNA3 vector that was similarly digested. Ligations were then transformed and grown on LB agar plates with ampicillin. Isolated colonies were cultured in LB broth and ampicillin for 24 hours and plasmids were isolated via the Qiagen Miniprep kits. Plasmids, encoding either PSAT1 gene or empty vector were verified by sequencing. Plasmids were then transfected into the MCF7 cell line using the Polyplus jetPRIME reagent according to manufacturer's protocol and clonal selection for PSAT1 expression was performed using geneticin.

\section{siRNA and shRNA Transfections}

PSAT1 shRNA and Control shRNA were purchased from Sigma. ShRNAs were transfected into LCC9 cells using the Polyplus jetPRIME reagent according to manufacturer's protocol. Clonal selection for gene silencing was performed using puromycin. The sequences for the shRNA and siRNA species are as follows: PSAT1 shRNA:

5'CCGGGCACTCAGTGTTGTTAGAGATCTCGAGATCTCTAACAACACTGAGTG $\begin{array}{llll}\text { CTTTTTG-3', } & \text { Control } & \text { 5'- }\end{array}$ CCGGCAACAAGATGAAGAGCACCAACTCGAGTTGGTGCTCTTCATCTTGTT GTTTTT-3', PHGDH RNAi: 5'-CGACAGGUUGCUGAAUGA-3', non-targeting pool RNAis: 5'-UGGUUUACAUGUCGACUAA-3', 5'-UGGUUUACAUGUUGUGUA-3', 5'-UGGUUUACAUGUUUUCUGA-3', 5'-UGGUUUACAUGUUUUCCUA-3'.

\section{Immunoblot Assays}

Whole-cell lysates were prepared in IP Lysis Buffer (Pierce) containing protease and phosphatase inhibitors. Proteins were separated on $10 \%$ SDS- 
polyacrylamide gels and transferred to PVDF. Membranes were blocked with 5\% non-fat dry milk in TBS-T and subsequently probed with 1:1000-fold dilution of antiPSAT1 (Proteintech, 10501-1-AP) or anti-PHGDH (Sigma HPA021241) antibodies for 16 hours at $4^{\circ}$. Washed membranes were then incubated with 1:5000 dilution of HRP-conjugated anti-rabbit or anti-mouse secondary antibodies. Protein detection was done by exposure to ECL Prime chemiluminescent reagent (GE Healthcare). Protein loading was assessed using anti- $\beta$-actin antibody (Sigma, A2228). Densitometry was performed via ImageJ.

\section{Stable Isotype Resolved Metabolomics (SIRM)}

MCF7 or LY2 cells were cultured in glucose-free IMEM supplemented with $5 \%$ dialyzed serum and $1 \mathrm{~g} / \mathrm{L}{ }^{13} \mathrm{C}_{6}$ - glucose (Sigma) for 48 hours. Metabolism was quenched via exposure to cold acetonitrile and cell metabolites were extracted using an acetonitrile/chloroform $/ \mathrm{H}_{2} \mathrm{O}$ mixture. Polar metabolites were subsequently lyophilized and analyzed by Liquid Chromatography Mass Spectrometry (LCMS) in collaboration with the Resource Center for Stable Isotype Resolved Metabolomics Core facility at the University of Kentucky.

\section{Treatments and Response}

Cells were treated with either 4-hydroxytamoxifen (4-OHT) or ICI 182,780 (ICl) for a period of four days [110]. Treatment response was measured by FluoReporter Blue Fluorometric dsDNA Quantitation Kit (Molecular Probes F2962). In brief, cells were plated at 5,000 (MCF-7) or 2,500 (LCC9) cells per well

on day 0 . On day 1 cells were treated with indicated concentrations of either 4$\mathrm{OHT}$ or $\mathrm{ICl}$. On day 4 the plates were collected and analyzed according to 
manufacturer's protocol. Treatment responses were calculated by percent decrease in fluorescence compared to the vehicle control.

\section{Quantitative-Real Time Polymerase-Chain Reaction}

MCF-7 or LCC9 cells were collected and total RNA was isolated using the RNeasy Mini Kit (Qiagen). One milligram of RNA was converted to cDNA using the High-Capacity RNA-to-cDNA kit (appliedbiosystems) according to the manufacture's protocol. Samples were then analyzed for qPCR via the TaqMan Fast Advanced (appliedbiosystems) system with human probes for PSAT1 (Hs00795278_mH), PHGDH (Hs00198333_m1), and ACTB (Hs01060665_g1).

\section{Kaplan Meier Analysis}

Retrospective analysis of specific gene expression on patient survival was performed by Kaplan-Meier analysis on de-identified patient clinical data obtained from either an IRB-approved institutional biorepository [111] or within the online database KM Plotter (kmplot.com) [112]. ER+BC patients treated with tamoxifen or any endocrine therapy were stratified for differential PSAT1 or PHGDH expression [113]. The hazard ratio (HR) with $95 \%$ confidence intervals and the logrank $p$ value are indicated. 


\section{Results}

\section{Clinical relevance of PSAT1 in ER+ patients treated with tamoxifen}

It has been previously observed that PSAT1 transcript and protein levels correlated with a poorer progression-free survival in ER+ patients treated with tamoxifen $[82,97]$. We initially validated these prior studies in two separate patient cohorts, including an institutional clinical population and individuals within the KM Plotter database. All patients presented with ER+BC and had undergone tamoxifen treatment as the sole endocrine therapy. In agreement, analysis of both patient cohorts revealed a negative correlation between higher PSAT1 expression and disease-free survival (Figure 24A) or relapse-free survival (Figure 24B). These results, as well as the previous studies, suggest that PSAT1 may be involved in tamoxifen resistance of $E R+B C$ patients.

\section{Manipulation of PSAT1 expression can alter sensitivity to tamoxifen therapy}

Prior studies have established in vitro models used to investigate the mechanisms that drive endocrine sensitive breast cancer to endocrine resistance [114]. One model system utilizes the sensitive MCF-7 ER+ cell line and its resistant derivatives. These derivative cell lines were generated by exposing parental MCF7 cells to increasing concentrations of endocrine therapies over an extended period of time (Figure 25A). Acquired from our collaborator, Dr. Carolyn Klinge, we employed this system to assess the contribution of PSAT1 and the SSP in general to tamoxifen sensitivity. We initially found that there was differential expression of PSAT1 between the resistant (LCC9 and LY2) and parental (MCF7) lines (Figure 25B). Transcript and protein analysis showed increasing levels of 


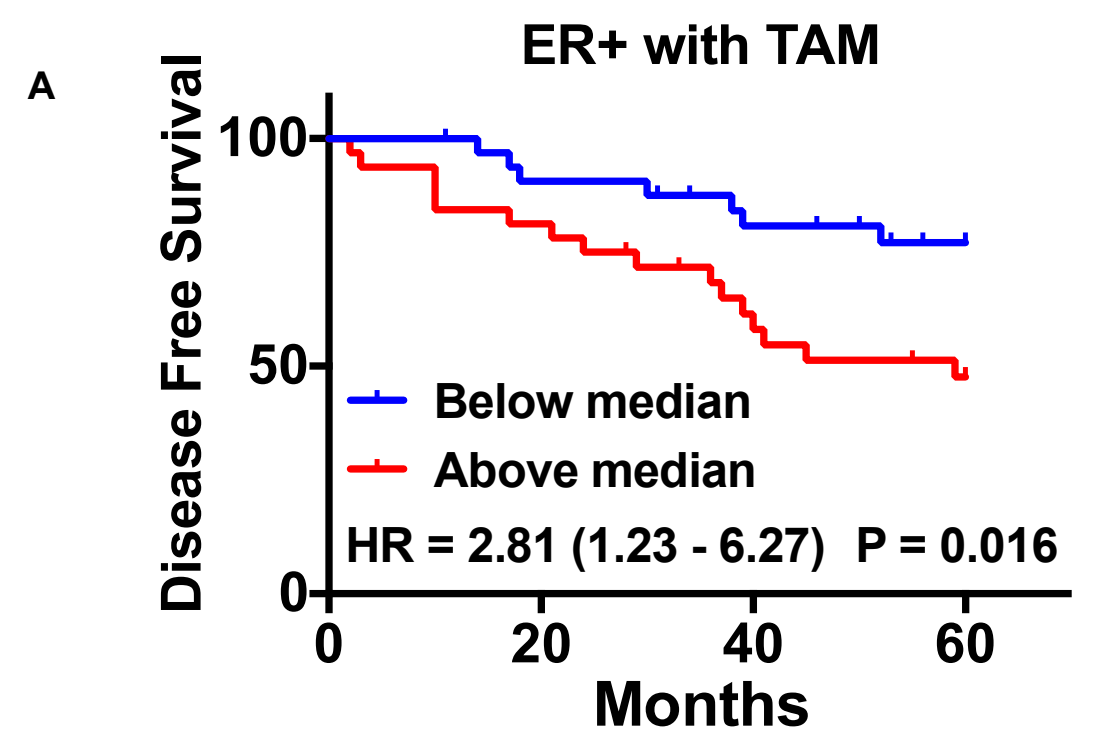

B

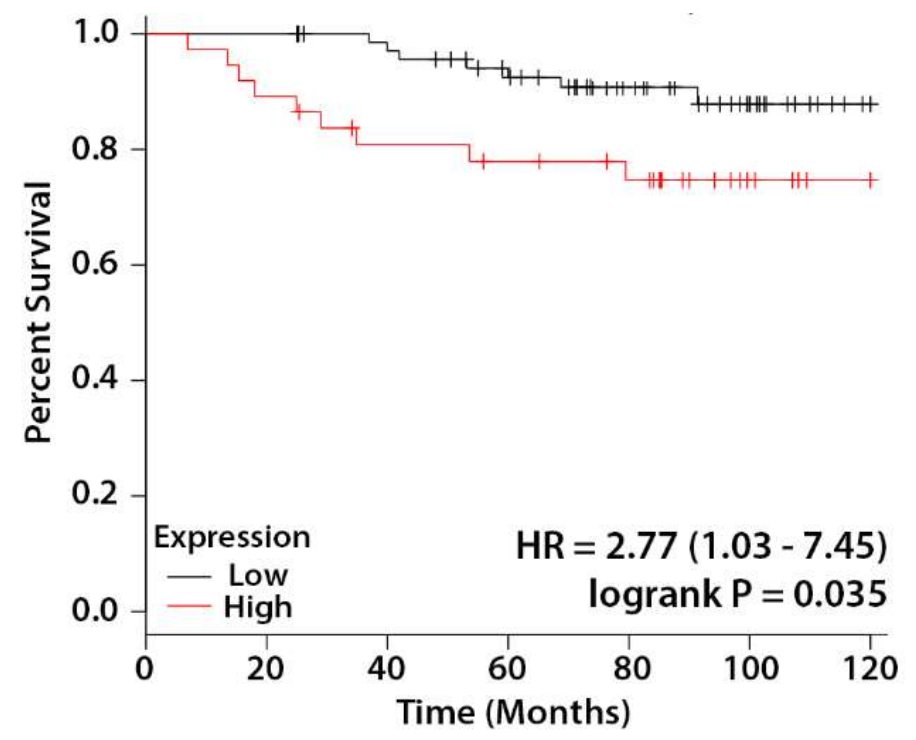

Figure 24: Clinical relevance of PSAT1 in Tamoxifen treated ER+BC. A) Disease-free survival of ER+ patients treated with tamoxifen stratified by above $(n=33)$ or below $(n=32)$ the median PSAT1 expression. Kaplan-Meier analysis was performed from previous transcriptomic analysis on a patient cohort within an institutional biorepository. B) Kaplan-Meier analysis of patient outcomes was done using KM Plotter database. Relapse-free survival of ER+ patients treated with tamoxifen stratified by low $(n=73)$ or high $(n=37)$ PSAT1 expression. 
A

\begin{tabular}{|l|lr|}
\hline \multicolumn{2}{|l|}{ Endocrine Sensitive } & Endocrine Resistant \\
\hline MCF-7 & LCC9 & LY2 \\
\hline
\end{tabular}

B

C

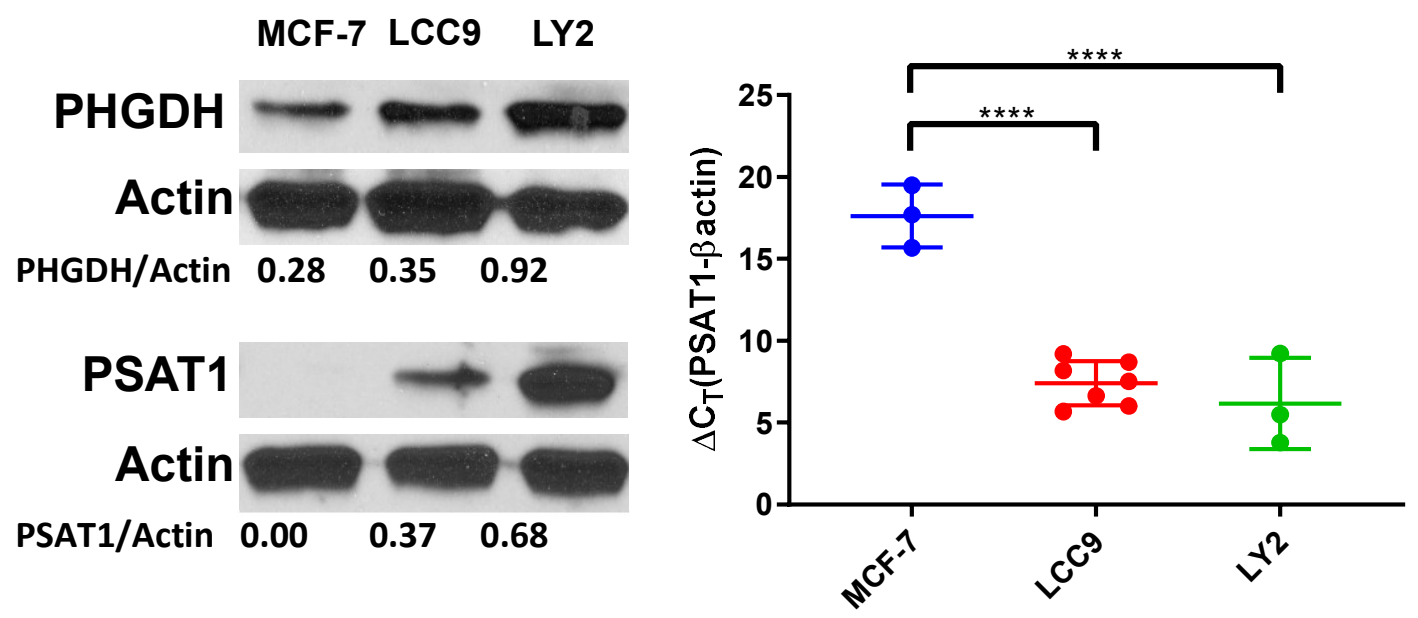

Figure 25: Differential PSAT1 expression between sensitive and resistant cell lines. A) Schematic summarizing the in vitro model system used in these studies. B) Representative western blots demonstrating the differential expression between the endocrine sensitive MCF-7 parental line and the endocrine resistant derivatives (LCC9 and LY2). Densitometry analysis was performed using ImageJ. C) Transcript levels, represented as $\Delta \mathrm{CT}$, of PSAT1 between the different cell lines. 
PSAT1 in the resistant cell types (Figure 25 B and C). Importantly, the MCF-7 sensitive cell line exhibits no PSAT1 expression as compared to the resistant lines. To determine whether the increase in enzyme expression corresponds to elevated SSP activity, we used stable isotope resolved metabolomics (SIRM) to evaluate glucose carbon incorporation into de novo serine production (Figure 26A). Using the LY2 cells, as they demonstrated the greatest enzyme expression, we found elevated SSP in the resistant cell line as evidence of greater levels of ${ }^{13} \mathrm{C}$ carbon from glucose into serine $(m+3$ serine), while activity in the MCF-7 line was below detectable limits (Figure 26B).

This differential expression of SSP enzymes, particularly PSAT1, between sensitive and resistant lines indicates that SSP activity may influence tamoxifen sensitivity. To investigate this potential metabolic consequence, we chose to alter PSAT1 levels in these cell models as endocrine sensitive MCF-7 cells do not express this SSP enzyme. First, PSAT1 was overexpressed in the MCF-7 cells (Figure 27A-B) to determine its effect on $4 \mathrm{OHT}$ response compared to the parental cells. Overexpression of PSAT1 in the MCF-7 cell line reduced the sensitivity of these cells at two different concentrations of 4-OHT (Figure 27C). Exposure to 100 or $500 \mathrm{nM} 4 \mathrm{OHT}$ led to $40-45 \%$ reduction in MCF-7 cell proliferation. Yet, PSAT1 expressing cells had reduced sensitivity to 4OHT (22$25 \%$ decrease respectively). Conversely, the contribution of PSAT1 to $4 \mathrm{OHT}$ resistance was also examined under loss of PSAT1 in the resistant LCC9 cell line. Using stable shRNA, PSAT1 was suppressed in the LCC9 cells (Figure28A) and resulted in a sensitization to $4 \mathrm{OHT}$ treatment (Figure 28B). Taken together, these 
A

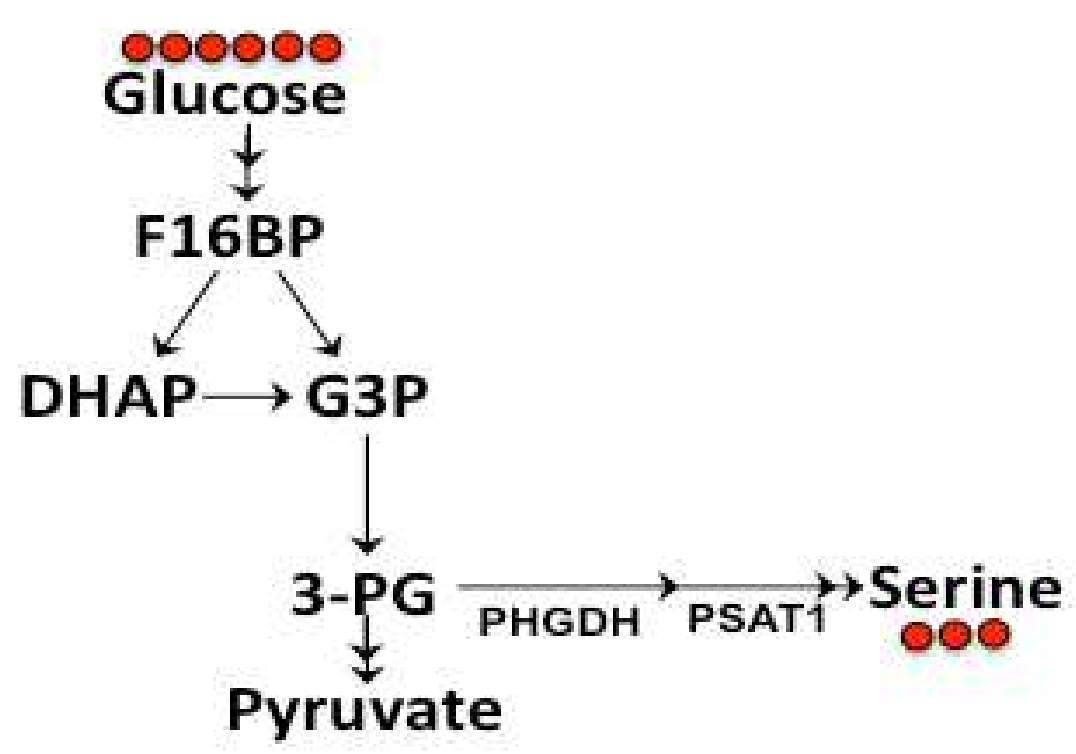

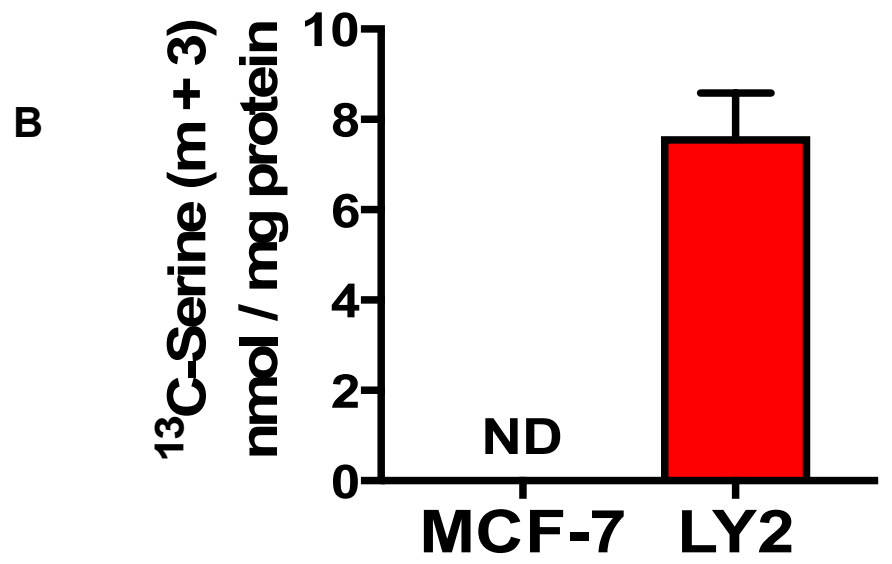

Figure 26: Metabolomic differences between endocrine sensitive and endocrine resistant cell types. A) Schematic demonstrating serine labeling patterns derived from ${ }^{13} \mathrm{C}$-Glucose labeling. B) ${ }^{13} \mathrm{C}$-Serine levels for the endocrine sensitive parental MCF-7 cell line and the endocrine resistant derivative (LY2) cell line. 
A
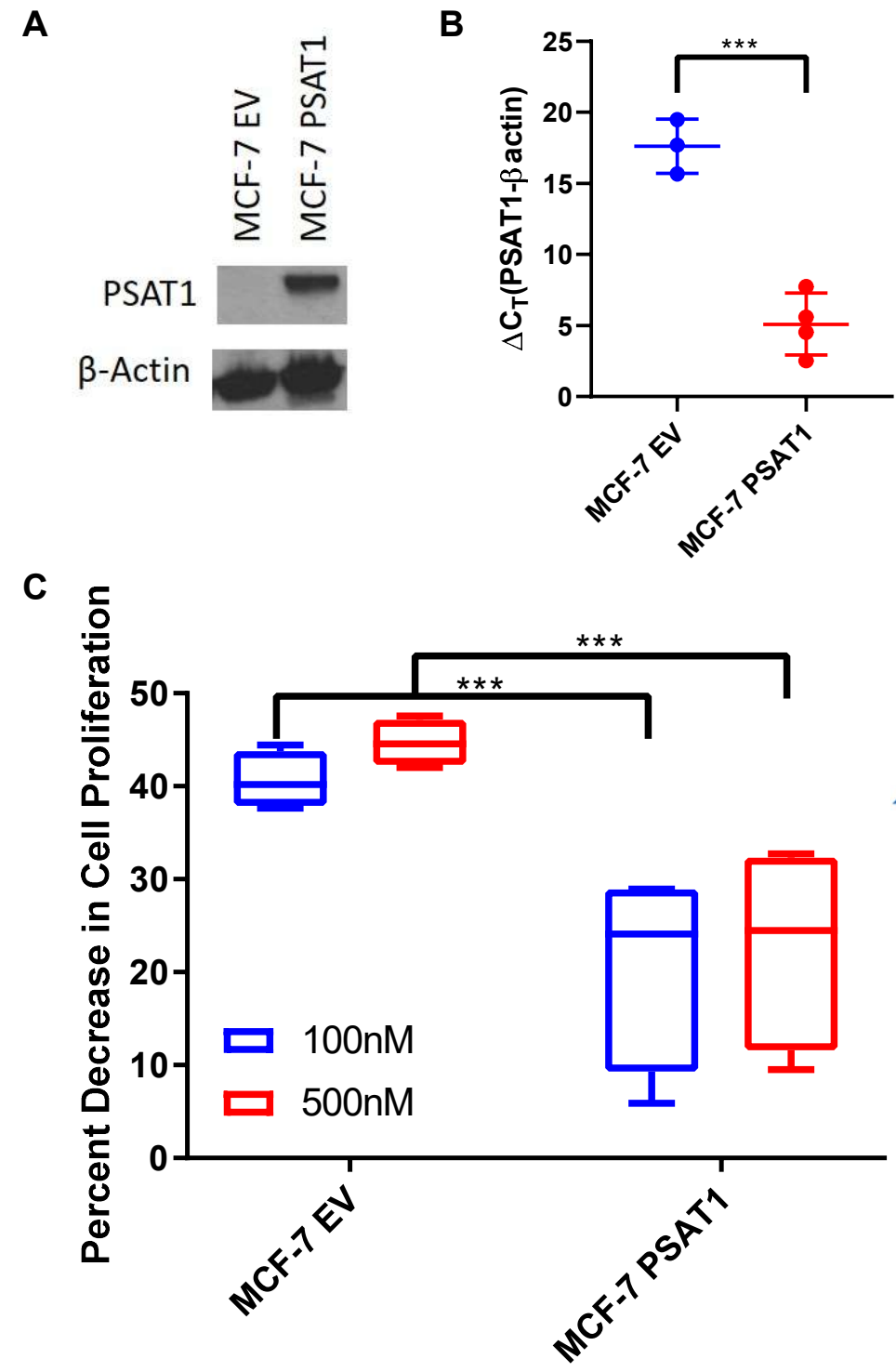

Figure 27: Overexpression of PSAT1 reduces sensitivity to 4hydroxytamoxifen. A) Representative western blot demonstrating the level of overexpression of PSAT1 in the MCF-7 cells. B) Transcript levels demonstrating the extent of PSAT1 overexpression in the MCF-7 cells. $p=0.0005$ as determine by unpaired t test. C) Response to $4 \mathrm{OHT}$, measured as percent decrease in cell proliferation, for both the MCF-7 empty vector and MCF-7 PSAT1 overexpression cell lines. $p=0.0006$ 100nM 4OHT, $p=0.0002$ 500nM 4OHT as determined by 2-way ANOVA with Tukey's comparison test. 
A

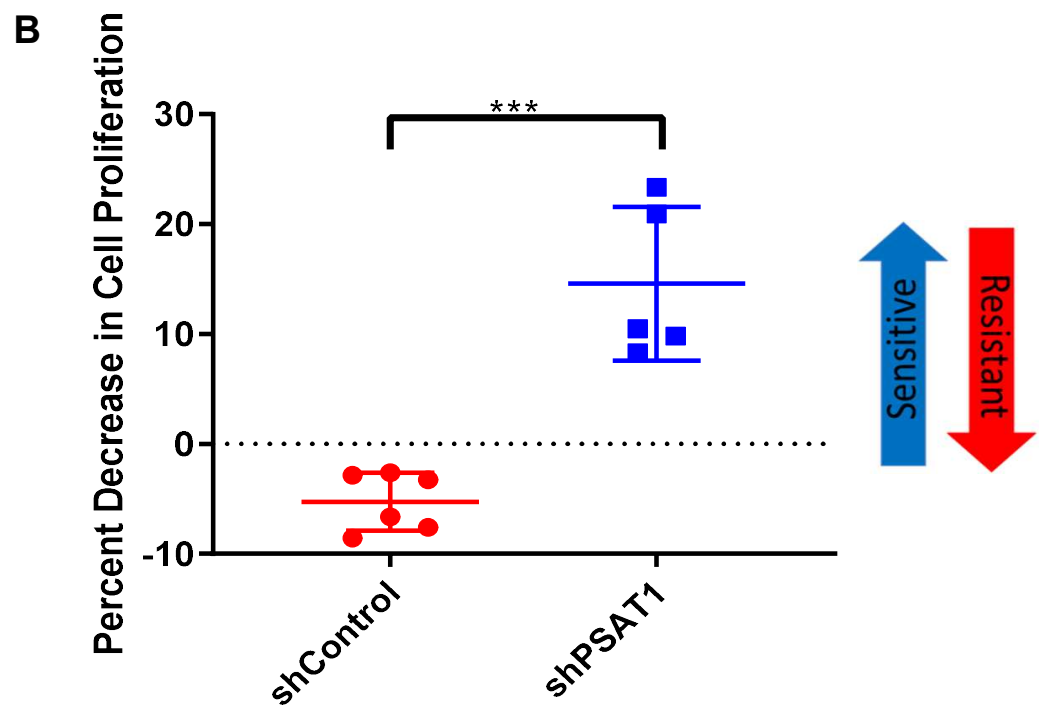

Figure 28: Suppression of PSAT1 increases sensitivity to 4hydroxytamoxifen. A) Representative western blot demonstrating the suppression of PSAT1 in the LCC9 cell line. B) Response to 500nM 4OHT, measured as percent decrease in cell proliferation, for both the LCC9 shControl and LCC9 shPSAT1 cell lines. $p=0.0001$ as determined by unpaired test. 
results indicate that PSAT1 and SSP activity may be a contributing factor in the resistance to tamoxifen of ER+ patients.

\section{Clinical relevance of PHGDH in ER+ patients treated with tamoxifen}

As part of the prior study on PSAT1 protein expression in clinical ER+BC samples, the authors also suggested that PHGDH might also be contributing to resistance in ER+ patients treated with tamoxifen [97]. This was attributed to the finding of increased expression of PHGDH in the tamoxifen treated patients that exhibited poorer clinical outcomes. However, no statistical or experimental analysis were performed to validate this potential relevance of PHGDH. Using KM plotter, we sought to determine if this observation could be associated with patient survival. Analyzing the same patient cohort used for PSAT1 (Figure 24), we found that patients with higher PHGDH expression also had significantly poorer relapsefree survival compared to patients with low PHGDH expression (Figure 29). Similar to PSAT1, suppression of PHGDH also sensitized LCC9 endocrine resistant cells to $4 \mathrm{OHT}$ treatment (Figure 30 ).

\section{Clinical correlation involving SSP enzymes and in vitro sensitivity to endocrine therapy is selective for tamoxifen}

Endocrine therapies encompass several different approaches to suppress estrogen signaling. Beyond $4 \mathrm{OHT}$, the pure antiestrogen Fulvestrant and aromatase inhibitors are frequently used in the clinic. Inevitably, patients will also become resistant to these endocrine therapies as well. We next wanted to determine whether SSP enzymes contributed to resistance to other endocrine treatments. Within in vitro experiments, we examined the effect of PSAT1 


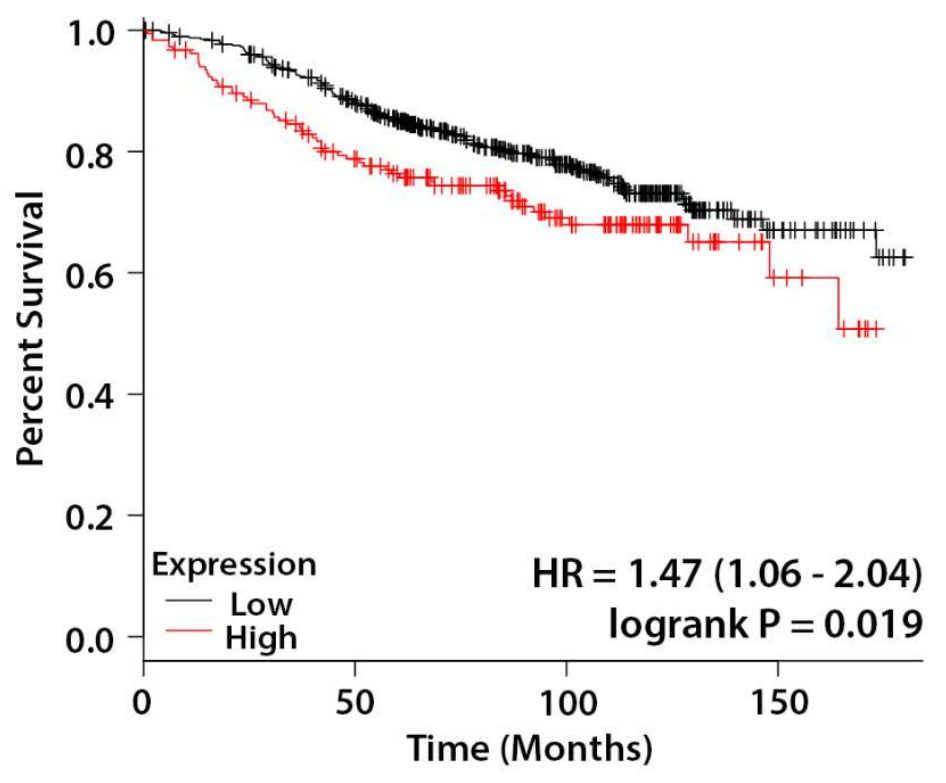

Figure 29: Clinical relevance of PHGDH in ER+ patients treated with tamoxifen. KM Plotter was used to determine relapse-free survival of ER+ patients treated with tamoxifen that was stratified by low $(n=486)$ or high $(n=184)$ PHGDH expression. 
A

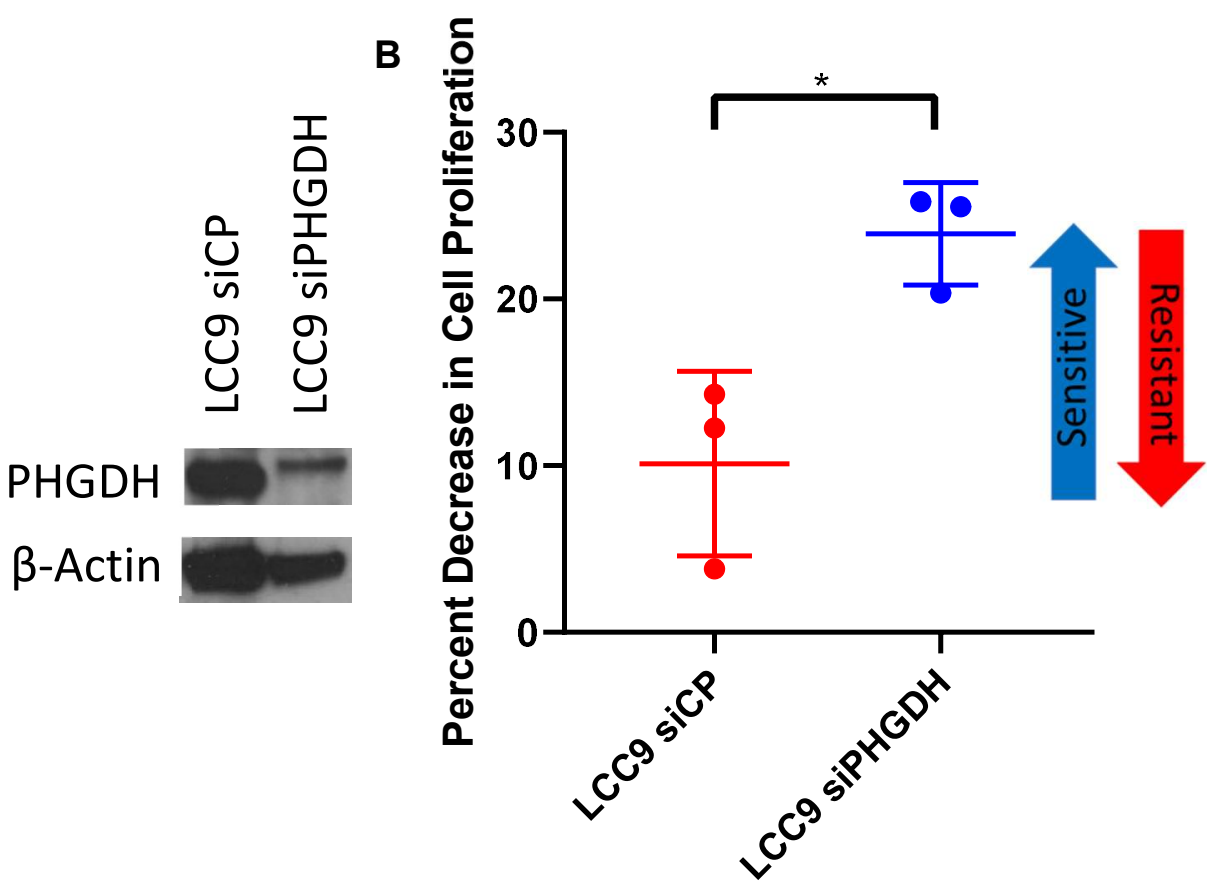

Figure 30: Suppression of PHGDH increases sensitivity to 4hydroxytamoxifen. A) Representative western blot demonstrating the suppression of PHGDH in the LCC9 cell line. B) Response to 500nM 4OHT, measured as percent decrease in cell proliferation, for both the LCC9 siRNA Control Pool (siCP) and LCC9 siPHGDH cell lines. $p=0.0197$ as determined by unpaired t test. 
suppression on sensitivity to ICI in LCC9 cells, as the LCC9 cell line is also resistant to ICI treatment [109]. We treated either control or PSAT1 suppressed cells with different concentrations of $\mathrm{ICl}$ and found no significant difference in cell proliferation (Figure 31A). To determine if this result extended to clinical data, we analyzed a patient cohort that was inclusive of ER+BC patients treated with any endocrine therapy. We then stratified these patients based on transcript levels of either PSAT1 (Figure 31B) or PHGDH (Figure 31C). When all endocrine therapies are included, significant association between high expression of the SSP enzymes and relapse-free survival is lost (Figure 31), contrary to what we observed with tamoxifen as the sole therapy (Figure 24, Figure 29). These data suggest that the contribution of PSAT1 or PHGDH to endocrine resistance may be limited to tamoxifen therapy. 

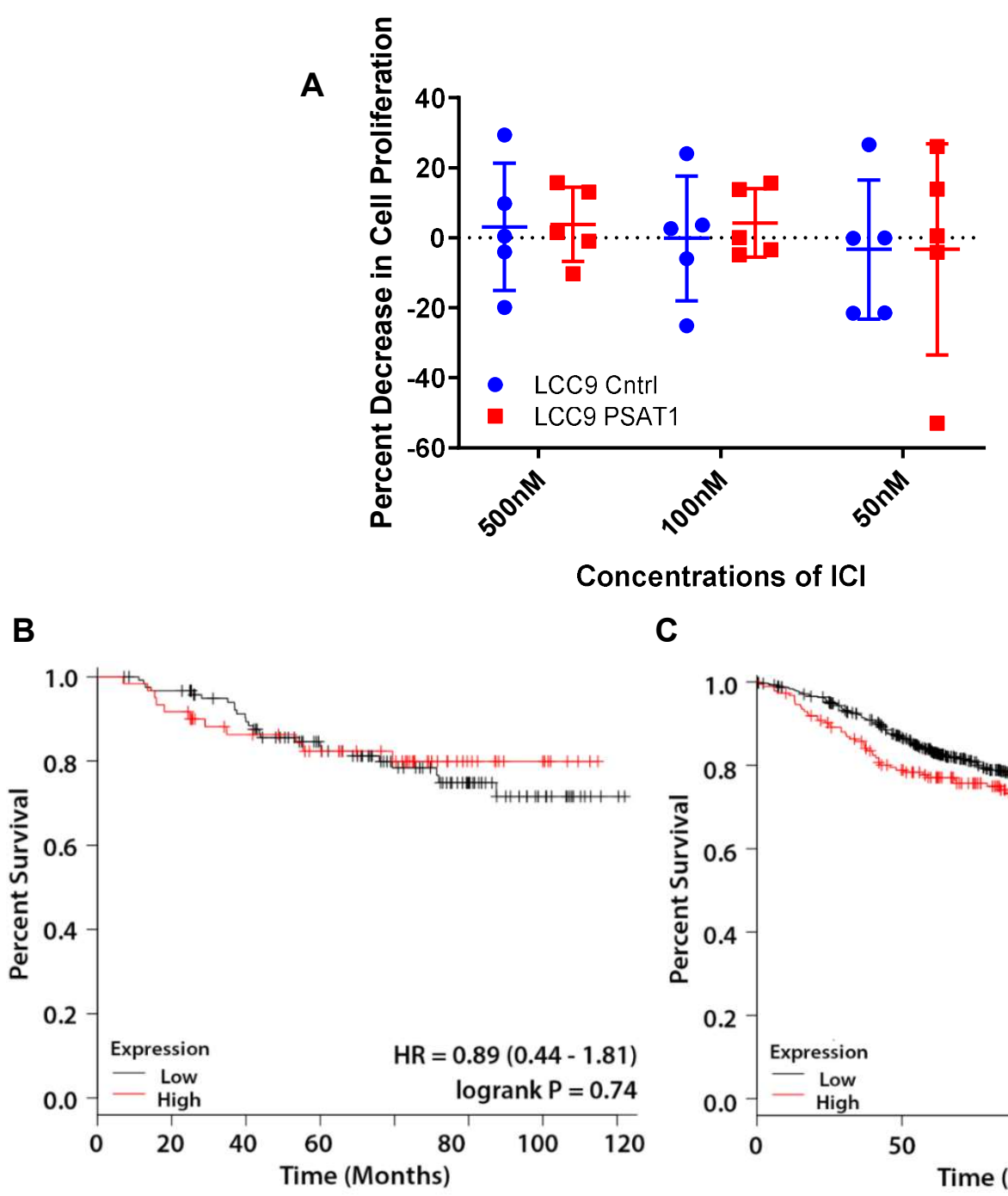

C

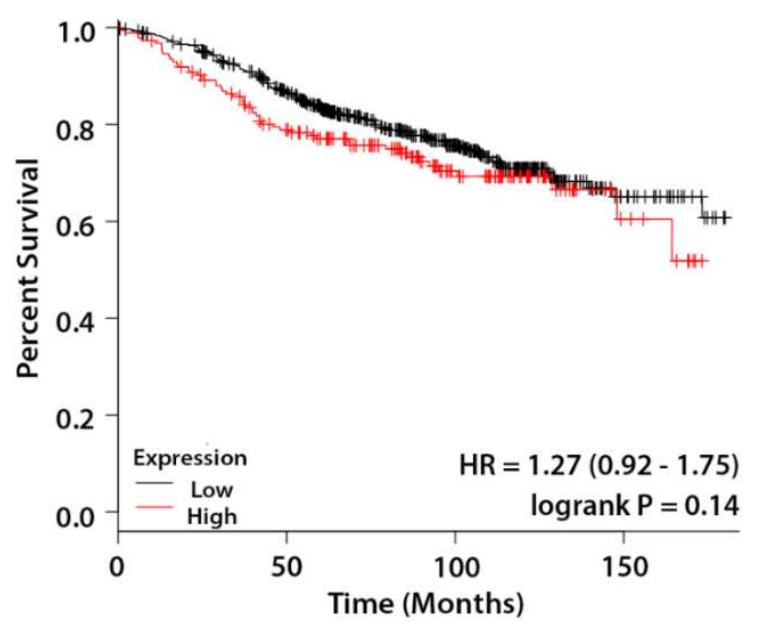

Figure 31: Correlation between SSP enzymes and relapse-free survival and in vitro sensitivity to endocrine therapy is selective for tamoxifen. A) LCC9 control of PSAT1 suppressed cells in response to ICI treatment at different concentrations. Response measured as percent decrease in cell proliferation. $p>0.999950 \mathrm{nM} \mathrm{ICl}$, $p=0.9774100 \mathrm{nM} \mathrm{ICl}, p>0.9999500 \mathrm{nM} \mathrm{ICl}$ as determined by 2-way ANOVA with Tukey's comparison test. B) Relapse-free survival of ER+ patients treated with all endocrine therapies stratified by low $(n=121)$ or high $(n=60)$ PSAT1 expression. C) Relapse-free survival of ER+ patients treated with all endocrine therapies stratified by low $(n=498)$ or high $(n=244)$ PHGDH expression. Kaplan-Meier analysis performed with KMPlotter database. 


\section{Discussion}

In this chapter we have demonstrated that both PSAT1 and PHGDH are correlated with poorer relapse-free survival in ER+ patients that were solely treated with tamoxifen. This agrees with previously published findings that implicated PSAT1 in tamoxifen resistance $[82,97]$. Furthermore, we showed that manipulation of PSAT1, either via overexpression in a tamoxifen sensitive model or suppression in a tamoxifen resistant model, can alter sensitivity to $4 \mathrm{OHT}$ treatment. In addition, loss of PHGDH was also shown to alter sensitivity to $4 \mathrm{OHT}$. These data indicate that the serine synthetic pathway contributes to tamoxifen resistance. Additionally, these effects seem to be selective for tamoxifen as both in vitro and clinical data failed to show any correlation between expression of serine synthetic enzymes and sensitivity to $\mathrm{ICl}$ or with inclusion of other endocrine therapies with respect to the $\mathrm{KM}$ analysis.

We speculate that the differences that we have observed between $4 \mathrm{OHT}$ and $\mathrm{ICl}$ treated cells could be related to their different mechanisms of action. Tamoxifen is a selective estrogen receptor modulator and its primary mode of action is through the binding to the estrogen receptor, competing with estrogens, resulting in inhibition of the estrogen driven pro-proliferative transcription program $[12,13]$. There is also evidence that tamoxifen can function independently of binding to the estrogen receptor via induction of transforming growth factor- $\beta$ (TGF- $\beta$ ) and through reduction of circulating insulin-like growth factor I (IGF-I) [13]. Fulvestrant is a "pure"-antiestrogen or a selective estrogen receptor downregulator [12]. Fulvestrant binds to the estrogen receptor, impairs its dimerization, 
and increases protein degradation and turn-over. This results in the complete attenuation of downstream estrogen signaling [12]. Importantly, in tamoxifen treated conditions, the estrogen receptor remains expressed; in contrast, during fulvestrant treated conditions there is a loss of the estrogen receptor [12]. This combined with our results suggest that tamoxifen resistance mediated by PSAT1 and PHGDH may be reliant upon either the maintenance of the estrogen receptor, its co-activators, or one of its gene targets. While the requirement for maintenance of ER as it relates to PSAT1 and PHGDH-mediated resistance would need further investigation, there is literature evidence to support this notion. MYC is a known target of estrogen receptor signaling and its function as well as its role in endocrine resistance requires maintenance of the ER [115].

Although these results suggest there might be a requirement for ER maintenance, the mechanism(s) by which PSAT1 and PHGDH are contributing to tamoxifen resistance is unclear. It could be through oncogenic activation of the SSP by proteins such as MYC, which has already been suggested to activate transcription of PSAT1 and PHGDH [52] and both (SSP and MYC) have been implicated in endocrine resistance $[82,97,116]$. Or the SSP-mediated resistance could be related to one of the metabolic consequences of de novo serine synthesis. Previous literature has suggested that glutamine is required for the proproliferative estrogen-driven signaling cascade in $E R+B C$ [115]. It has also been suggested that while uptake of glutamine is independent of estrogen signaling, this process may also require maintenance of ER expression [115], similar to what our results suggest for PSAT1 and PHGDH-mediated resistance. Additionally, PSAT1 
is responsible for the second processing step of intracellular glutamine as it converts glutamate to a-ketogluterate [28], which connects glutamine consumption, the SSP, and endocrine resistance to maintenance of ER expression. Alternatively, epigenetic changes have also been proposed as a mechanism for tamoxifen resistance [22]. These changes include increases in hypomethylated promoter regions of oncogenic genes and hypermethylated promoter regions of tumor suppressors [22]. There is also evidence to suggest that in breast cancer the ER cofactors are under epigenetic control and that dysregulation of these cofactors is correlated with resistance to tamoxifen therapy [22]. As stated, PSAT1 and PHGDH are both members of the serine synthetic pathway along with PSPH. This pathway diverges from the glycolytic pathway at 3-phosphoglycerate for de novo synthesis of serine [28]. Downstream, serine can be used in one carbon metabolism to generate s-adenosyl-methionine (SAM) [117]. SAM is the methyl donor for the methylation of nucleic acids, proteins, and lipids and there has been evidence to support that the levels of serine dictate the levels of SAM [117].

Given the literature and our results, we hypothesize that PSAT1 and PHGDH are contributing to tamoxifen resistance through de novo serine synthesis by both processing of glutamine and the downstream generation of SAM and subsequent epigenetic changes but further investigation into this area would be needed to confirm that this is the mechanism in which the serine synthetic pathway is contributing to resistance (Figure 32). In addition, it would be interesting if treatment with PHGDH inhibitors $[86,118]$ would be able to recapitulate the effects 


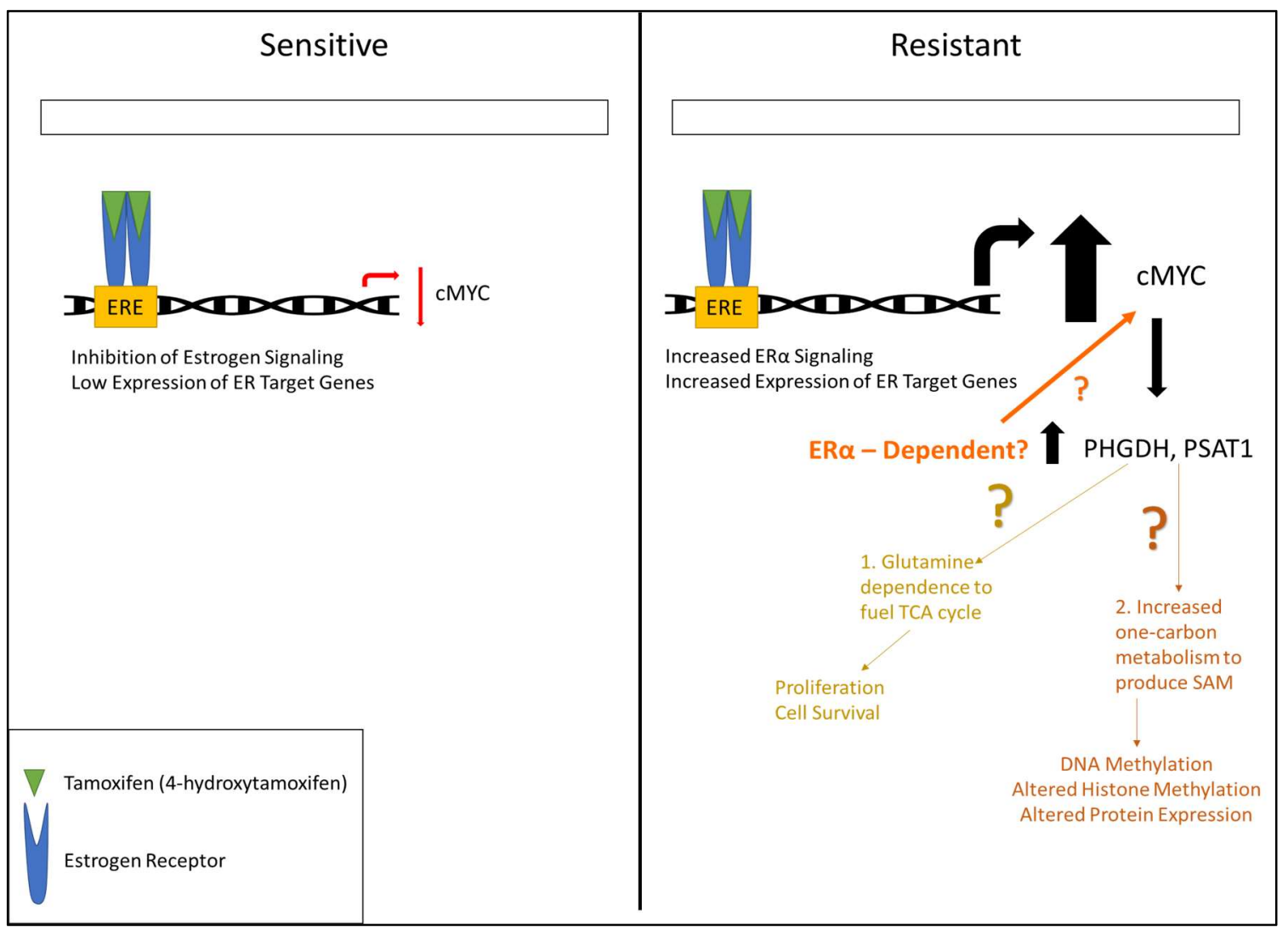

Figure 32: Model of SSP contribution to endocrine resistance. This model represents the suggested mechanisms by which PSAT1 and PHGDH are contributing to tamoxifen resistance in $\mathrm{ER}+\mathrm{BC}$. In sensitive conditions there is an inhibition of estrogen signaling and low expression of ER target genes. In resistant condition there is increased ERa signaling, despite a tamoxifen (4hydroxytamoxifen) occupied ER, and increased expression of ER target genes. One target gene, cMYC, is increased in resistant conditions and has been shown to increase expression of PSAT1 and PHGDH but it is unknown if this increase is dependent on ERa. The upregulation of SSP enzymes can contribute to resistance via either glutamine dependence to fuel the TCA cycle or through increased one-carbon metabolism to produce SAM. 
of genetic suppression of PHGDH and PSAT1 that we have observed in this chapter. Those results might suggest a combination therapy that may reduce resistance and prolong efficacy to tamoxifen therapy. 


\section{CHAPTER 4}

\section{CONCLUSION}

In summary, this body of work has demonstrated that PSAT1 contributes to the progression of breast cancer in the contexts of TNBC metastasis and ER+BC tamoxifen resistance. We have shown that suppression of PSAT1 is able to diminish metastatic characteristics in vitro and that loss of PHGDH does not recapitulate these results. We have also shown that suppression of PSAT1 inhibits tumor nodule formation in an in vivo model of experimental metastasis and that this inhibition is not due to a loss of tumorgenicity. The results from our TNBC studies suggest a non-canonical role for PSAT1 that selectively inhibits the metastatic characteristics of "serine synthesis-independent" TNBC (Chapter 2).

We have also demonstrated a correlation between higher expression levels of PSAT1 and poorer progression-free survival in two distinct ER+BC patient cohorts. In addition, we have shown that manipulation of PSAT1 and PHGDH can alter sensitivity to tamoxifen treatment in an established sensitive and resistant breast cancer cell model system. Our work indicates that these results are due to changes in de novo serine synthesis as clinically high expression of PHGDH also correlates with poorer progression-free survival and there is increased flux of glucose to serine in endocrine resistant cells. We also demonstrated that these effects of the SSP enzymes appear to be selective for $4 \mathrm{OHT}$ treatment as 
disruption of the SSP through PSAT1 loss did not alter fulvestrant sensitivity in two tamoxifen resistant cell lines. In addition, all clinical significance regarding inverse correlation of PSAT1 and PHGDH with relapse-free survival in ER+BC was lost upon inclusion of patients treated with any endocrine therapy (Chapter 3).

My data suggests that the functional roles of PSAT1 as it contributes to either metastasis or resistance varies. In the TNBC component of this dissertation, PSAT1 is acting independently of its role in serine synthesis as we established that PHGDH suppression did not recapitulate PSAT1 suppression. However, in the ER+BC portion of this dissertation it seems that the contribution of PSAT1 to tamoxifen resistance is associated with its role in serine synthesis as clinically both PSAT1 and PHGDH significantly correlated with poorer progression-free survival. These results, while conclusive, leave some remaining questions. Primarily, how does the expression of a single protein, PSAT1, promote two distinct processes (metastasis or endocrine resistance) via two separate mechanisms (canonical vs. non-canonical)? In addition, what dictates either canonical or non-canonical function of PSAT1 and are these functions mutually exclusive? Lastly, is this phenomenon driven exclusively by the proteome differences between TNBC and ER+ tamoxifen resistant breast cancer subtypes?

It is known that there are subtype-specific expression differences for PSAT1 in other cancer types. It has been shown that PSAT1 is higher in certain ovarian cancer subtypes and that PSAT1 expression can be used as a biomarker to determine the subtype of ovarian cancer a patient has [78]. In addition, PSAT1 expression also varies in thyroid cancer subtypes [57]. PSAT1 expression in breast 
cancer is also known to vary by subtype. PSAT1 protein expression is greater in ER- compared to the ER+BC $[84,87,94,95]$. The lack of expression of PSAT1 in $\mathrm{ER}+\mathrm{BC}$ is mainly due to hypermethylation of the PSAT1 promoter within this subtype [94]. This suggests that there might be an ER-dependent silencing of PSAT1 within the ER+ subtype that is lost in tumors that are ER-. Previous literature would also suggest that this potential ER-dependent regulation is specific for PSAT1 and not a regulatory mechanism for the SSP as the differences in promoter methylation are specific for PSAT1 $[82,94,95]$ and that both ER+ and ERtumors express PHGDH [87].

The role of PSAT1 in metastasis that we have defined in this body of work is in a specific subset of TNBC that is "serine-synthesis independent". This subset allowed us to determine if there was a PSAT1 specific role in metastasis that was independent from its role in de novo serine synthesis. My data suggests that there is a selective role for PSAT1 in breast cancer metastasis. Other groups have also found an association between PSAT1 and metastasis that was not found with other members of the SSP [85]. This specific association between PSAT1 and metastasis has also been observed in several other cancer types. This has been observed in nasopharyngeal carcinoma [65] where higher expression of PSAT1 correlated with poorer local recurrence-free and distant metastatic-free survival. Additionally, higher PSAT1 expression was correlated with metastatic characteristics $[57,58]$ as well as lymph-node metastasis and distant metastasis in esophageal squamous cell carcinoma [72]. While in the report on nasopharyngeal carcinoma no mechanism was suggested, the findings in esophageal squamous 
cell carcinoma also indicated a non-canonical function of PSAT1 in order to upregulate the expression/activity of GSK3 $\beta$ and Snail that promoted metastatic characteristics in their in vitro studies [72].

In addition, there have been numerous accounts of de novo serine synthesis implicated in resistance. This has been demonstrated in multiple myeloma where higher activity of SSP enzymes was observed in resistant cell types and suggested that expression of the SSP enzymes could serve as a biomarker for Bortezomib resistance [69]. There has also been a link established between SSP and resistance to the BRAF inhibitor Vemurafenib [68]. This was observed in melanoma, pancreatic cancer, and non-small cell lung cancer in which SSP enzyme expression was higher in resistant cell types which resulted in increased proliferation [68]. Our work, as well as previous work [82,97], demonstrates that there is a role for the SSP in resistance to tamoxifen in ER+BC as higher expression of both PSAT1 and PHGDH correlate to poorer progressionfree survival.

However, the question remains is the canonical and non-canonical function of PSAT1 mutually exclusive and more interestingly, would the metastatic characteristics of endocrine resistant cells be solely affected by suppression of PSAT1? While these experiments were not performed as a part of this work, there is some literature to suggest that these functions are not mutually exclusive and that PSAT1 still contributes to metastasis independently of function in de novo serine synthesis. Studies completed in models of colon cancer demonstrate that there is increased activity of the SSP in colon tumors compared to normal colon 
tissue [74], especially in patients that were resistant to chemotherapies [75]. However, PSAT1 was the singular most upregulated protein in colorectal carcinoma patients [75] and specifically increased PSAT1 expression correlated with metastasis in resistant patients [73]. We have previously discussed that PSAT1 correlates with tamoxifen resistance [82] and that upregulation of PHGDH was also found in tamoxifen resistant patients [97] as this was the foundation for the studies completed in chapter 3 . However, we did not address metastasis in our ER+BC studies. Metastasis and its correlation to PSAT1 and PHGDH expression in the ER+ tamoxifen resistant setting has been previously studied [97]. In part, they found that PSAT1 and not PHGDH correlated with metastasis in ER+ patients who are tamoxifen resistant [97]. Both of these findings indicate that PSAT1 can simultaneously function within serine synthesis to promote tamoxifen resistance, as we have demonstrated, and independently contribute to metastasis as observed in our TNBC experiments.

The question of what dictates the function of PSAT1 remains unknown. This could be related to how PSAT1 expression is regulated and/or whether or not downstream effectors of PSAT1 that are responsible for either metastasis or endocrine resistance are present. Loss of miRNA-dependent suppression of PSAT1 resulted in increased protein expression and correlated with metastatic disease in two different cancer types $[57,58]$. Increased demand for serine and the resultant one-carbon metabolism products upregulate PSAT1 and de novo serine synthesis as a means of ensuring tumor cell survival whilst undergoing therapeutic treatment leading to the resistant phenotypes observed. In both of these instances, 
the upregulation of PSAT1 and the resultant function was determined by a combination of events that included additional steps and/or proteins, thus PSAT1 may be the driver of metastasis and/or endocrine resistance but the determination of which process occurs could be decided by which downstream pathways ensure the survival of the cancer cell.

Together, my data suggest that the metastatic phenotype is related to a function of PSAT1 that is independent from its role in serine synthesis. It also underscores that therapy resistance is reliant upon the serine synthetic pathway which is consistent with previous findings that altered methylation patterns correlate with resistance and that de novo synthesized serine is used in onecarbon metabolism. Despite differences in functional roles and reliance on serine, this body of work has established that PSAT1 contributes to the progression of breast cancer through promoting metastasis in TNBC and resistance to tamoxifen in $\mathrm{ER}+\mathrm{BC}$. 


\section{REFERENCES}

1. Siegel RL, Miller KD, Jemal A (2019) Cancer statistics, 2019. CA Cancer J Clin 69 (1):7-34. doi:10.3322/caac.21551

2. Howlader N NA, Krapcho M, Miller D, Bishop K, Kosary CL, Yu M, Ruhl J, Tatalovich Z, Mariotto A, Lewis DR, Chen HS, Feuer EJ, Cronin KA (eds) (2017) SEER Cancer Statistics Review. National Cancer Institute Bethesda, MD 19752014

3. Society AC (2018) Breast Cancer Facts \& Figures 2017-2018. Atlanta: American Cancer Society, INC 2017

4. Bianchini G, Balko JM, Mayer IA, Sanders ME, Gianni L (2016) Triple-negative breast cancer: challenges and opportunities of a heterogeneous disease. Nat Rev Clin Oncol 13 (11):674-690. doi:10.1038/nrclinonc.2016.66

5. Al-Mahmood S, Sapiezynski J, Garbuzenko OB, Minko T (2018) Metastatic and triple-negative breast cancer: challenges and treatment options. Drug Deliv Transl Res 8 (5):1483-1507. doi:10.1007/s13346-018-0551-3

6. Kumar P, Aggarwal R (2016) An overview of triple-negative breast cancer. Arch Gynecol Obstet 293 (2):247-269. doi:10.1007/s00404-015-3859-y

7. Yip CH, Rhodes A (2014) Estrogen and progesterone receptors in breast cancer. Future Oncol 10 (14):2293-2301. doi:10.2217/fon.14.110

8. Dai X, Li T, Bai Z, Yang Y, Liu X, Zhan J, Shi B (2015) Breast cancer intrinsic subtype classification, clinical use and future trends. Am J Cancer Res 5 (10):2929-2943

9. Schmid P (2017) Endocrine Therapeutic Strategies for Patients with Hormone Receptor-positive Advanced Breast Cancer. European Oncology \& Haematology $13(2): 127-133$

10. Bai Z, Gust R (2009) Breast cancer, estrogen receptor and ligands. Arch Pharm (Weinheim) 342 (3):133-149. doi:10.1002/ardp.200800174

11. Clarke R, Tyson JJ, Dixon JM (2015) Endocrine resistance in breast cancer-An overview and update. Mol Cell Endocrinol 418 Pt 3:220-234. doi:10.1016/j.mce.2015.09.035

12. Robertson JF (2001) ICI 182,780 (Fulvestrant)--the first oestrogen receptor down-regulator--current clinical data. $\mathrm{Br} J$ Cancer 85 Suppl 2:11-14. doi:10.1054/bjoc.2001.1982

13. Sporn MB LS (2003) Agents for Chemoprevention and Their Mechanism of Action. In: Kufe DW PR, Weichselbaum RR, et al. (ed) Holland-Frei Cancer Medicine. 6th edn., Hamilton (ON): BC Decker,

14. O'Shaughnessy J (2005) Extending Survival with Chemotherapy in Metastatic Breast Cancer. The Oncologist 10 (3):20-29

15. Gupta GP, Massague J (2006) Cancer metastasis: building a framework. Cell 127 (4):679-695. doi:10.1016/j.cell.2006.11.001 
16. Jin X, Mu P (2015) Targeting Breast Cancer Metastasis. Breast Cancer (Auckl) 9 (Suppl 1):23-34. doi:10.4137/BCBCR.S25460

17. Kimbung S, Loman N, Hedenfalk I (2015) Clinical and molecular complexity of breast cancer metastases. Semin Cancer Biol 35:85-95. doi:10.1016/j.semcancer.2015.08.009

18. Daves MH, Hilsenbeck SG, Lau CC, Man TK (2011) Meta-analysis of multiple microarray datasets reveals a common gene signature of metastasis in solid tumors. BMC Med Genomics 4:56. doi:10.1186/1755-8794-4-56

19. Lambert AW, Pattabiraman DR, Weinberg RA (2017) Emerging Biological Principles of Metastasis. Cell 168 (4):670-691. doi:10.1016/j.cell.2016.11.037 20. Mego M, Mani SA, Cristofanilli M (2010) Molecular mechanisms of metastasis in breast cancer--clinical applications. Nat Rev Clin Oncol 7 (12):693-701. doi:10.1038/nrclinonc.2010.171

21. Bogenrieder T, Herlyn M (2003) Axis of evil: molecular mechanisms of cancer metastasis. Oncogene 22 (42):6524-6536. doi:10.1038/sj.onc.1206757

22. Dixon JM (2014) Endocrine Resistance in Breast Cancer. New Journal of Science 2014:1-27. doi:10.1155/2014/390618

23. Hanahan D, Weinberg RA (2011) Hallmarks of cancer: the next generation. Cell 144 (5):646-674. doi:10.1016/j.cell.2011.02.013

24. Vander Heiden MG, DeBerardinis RJ (2017) Understanding the Intersections between Metabolism and Cancer Biology. Cell 168 (4):657-669. doi:10.1016/j.cell.2016.12.039

25. Warburg O, Wind F, Negelein E (1927) The Metabolism of Tumors in the Body. J Gen Physiol 8 (6):519-530. doi:10.1085/jgp.8.6.519

26. Vander Heiden MG, Cantley LC, Thompson CB (2009) Understanding the Warburg effect: the metabolic requirements of cell proliferation. Science 324 (5930):1029-1033. doi:10.1126/science.1160809

27. Amelio I, Cutruzzola F, Antonov A, Agostini M, Melino G (2014) Serine and glycine metabolism in cancer. Trends Biochem Sci 39 (4):191-198. doi:10.1016/j.tibs.2014.02.004

28. Mattaini KR, Sullivan MR, Vander Heiden MG (2016) The importance of serine metabolism in cancer. J Cell Biol 214 (3):249-257. doi:10.1083/jcb.201604085 29. Locasale JW (2013) Serine, glycine and one-carbon units: cancer metabolism in full circle. Nat Rev Cancer 13 (8):572-583. doi:10.1038/nrc3557

30. Sullivan MR, Vander Heiden MG (2017) When cancer needs what's nonessential. Nat Cell Biol 19 (5):418-420. doi:10.1038/ncb3523

31. Kalhan SC, Hanson RW (2012) Resurgence of serine: an often neglected but indispensable amino Acid. J Biol Chem 287 (24):19786-19791. doi:10.1074/jbc.R112.357194

32. Luo J (2011) Cancer's sweet tooth for serine. Breast Cancer Res 13 (6):317. doi:10.1186/bcr2932

33. DeBerardinis RJ (2011) Serine metabolism: some tumors take the road less traveled. Cell Metab 14 (3):285-286. doi:10.1016/j.cmet.2011.08.004

34. Uhlen M, Fagerberg L, Hallstrom BM, Lindskog C, Oksvold P, Mardinoglu A, Sivertsson A, Kampf C, Sjostedt E, Asplund A, Olsson I, Edlund K, Lundberg E, 
Navani S, Szigyarto CA, Odeberg J, Djureinovic D, Takanen JO, Hober S, Alm T, Edqvist PH, Berling H, Tegel H, Mulder J, Rockberg J, Nilsson P, Schwenk JM, Hamsten M, von Feilitzen K, Forsberg M, Persson L, Johansson F, Zwahlen M, von Heijne G, Nielsen J, Ponten F (2015) Proteomics. Tissue-based map of the human proteome. Science 347 (6220):1260419. doi:10.1126/science.1260419 35. de Koning TJ, Snell K, Duran M, Berger R, Poll-The BT, Surtees R (2003) Lserine in disease and development. Biochem J 371 (Pt 3):653-661. doi:10.1042/BJ20021785

36. Yamasaki MY, Keiko; Furuya, Shigeki; Mitoma, Junya; Hirabayashi, Yoshio; Watanabe, Masahiko (2001) 3-Phosphoglycerate Dehydrogenase, a Key Enzyme forl-Serine Biosynthesis, Is Preferentially Expressed in the Radial Glia/Astrocyte Lineage and Olfactory Ensheathing Glia in the Mouse Brain. Journal of Neuroscience 21 (19):7691-7704. doi: https://doi.org/10.1523/JNEUROSCI.21-1907691.2001

37. Hart CE, Race V, Achouri Y, Wiame E, Sharrard M, Olpin SE, Watkinson J, Bonham JR, Jaeken J, Matthijs G, Van Schaftingen E (2007) Phosphoserine aminotransferase deficiency: a novel disorder of the serine biosynthesis pathway. Am J Hum Genet 80 (5):931-937. doi:10.1086/517888

38. Davis JL, Fallon HJ, Morris HP (1970) Two enzymes of serine metabolism in rat liver and hepatomas. Cancer Res 30 (12):2917-2920

39. Snell K (1984) Enzymes of serine metabolism in normal, developing and neoplastic rat tissues. Adv Enzyme Regul 22:325-400

40. Baek JY, Jun DY, Taub D, Kim YH (2003) Characterization of human phosphoserine aminotransferase involved in the phosphorylated pathway of Lserine biosynthesis. Biochem J 373 (Pt 1):191-200. doi:10.1042/BJ20030144

41. Hwang IY, Kwak S, Lee S, Kim H, Lee SE, Kim JH, Kim YA, Jeon YK, Chung DH, Jin X, Park S, Jang H, Cho EJ, Youn HD (2016) Psat1-Dependent Fluctuations in alpha-Ketoglutarate Affect the Timing of ESC Differentiation. Cell Metab 24 (3):494-501. doi:10.1016/j.cmet.2016.06.014

42. Baek JYJ, D.Y.; Taub, D.; Kim, Y.H. (2003) Characterization of human phosphoserine aminotransferase involved in the phosphorylated pathway of Lserine biosynthesis. Biochemistry Journal (373):191-200

43. Stelzer G RR, Plaschkes I, Zimmerman S, Twik M, Fishilevich S, Iny Stein T, Nudel R, Lieder I, Mazor Y, Kaplan S, Dahary D, Warshawsky D, Guan - Golan Y, Kohn A, Rappaport N, Safran M, and Lancet D (2016) The GeneCards Suite: From Gene Data Mining to Disease Genome Sequence Analysis. Current Protocols in Bioinformatics 54 (1):1.30.31-31.30.33. doi:https://doi.org/10.1002/cpbi.5

44. Svoboda LK, Teh SSK, Sud S, Kerk S, Zebolsky A, Treichel S, Thomas D, Halbrook CJ, Lee HJ, Kremer D, Zhang L, Klossowski S, Bankhead AR, Magnuson B, Ljungman M, Cierpicki T, Grembecka J, Lyssiotis CA, Lawlor ER (2018) Menin regulates the serine biosynthetic pathway in Ewing sarcoma. J Pathol 245 (3):324336. doi:10.1002/path.5085

45. Ding J, Li T, Wang X, Zhao E, Choi JH, Yang L, Zha Y, Dong Z, Huang S, Asara JM, Cui H, Ding HF (2013) The histone H3 methyltransferase G9A epigenetically activates the serine-glycine synthesis pathway to sustain cancer cell 
survival and proliferation. Cell Metab 18 (6):896-907. doi:10.1016/j.cmet.2013.11.004

46. Zhao E, Ding J, Xia Y, Liu M, Ye B, Choi JH, Yan C, Dong Z, Huang S, Zha Y, Yang L, Cui H, Ding HF (2016) KDM4C and ATF4 Cooperate in Transcriptional Control of Amino Acid Metabolism. Cell Rep 14 (3):506-519. doi:10.1016/j.celrep.2015.12.053

47. DeNicola GM, Chen PH, Mullarky E, Sudderth JA, Hu Z, Wu D, Tang H, Xie Y, Asara JM, Huffman KE, Wistuba, II, Minna JD, DeBerardinis RJ, Cantley LC (2015) NRF2 regulates serine biosynthesis in non-small cell lung cancer. Nat Genet 47 (12):1475-1481. doi:10.1038/ng.3421

48. Riscal R, Schrepfer E, Arena G, Cisse MY, Bellvert F, Heuillet M, Rambow F, Bonneil E, Sabourdy F, Vincent C, Ait-Arsa I, Levade T, Thibaut P, Marine JC, Portais JC, Sarry JE, Le Cam L, Linares LK (2016) Chromatin-Bound MDM2 Regulates Serine Metabolism and Redox Homeostasis Independently of p53. Mol Cell 62 (6):890-902. doi:10.1016/j.molcel.2016.04.033

49. Gao S, Ge A, Xu S, You Z, Ning S, Zhao Y, Pang D (2017) PSAT1 is regulated by ATF4 and enhances cell proliferation via the GSK3beta/beta-catenin/cyclin D1 signaling pathway in ER-negative breast cancer. J Exp Clin Cancer Res 36 (1):179. doi:10.1186/s13046-017-0648-4

50. Amelio I, Markert EK, Rufini A, Antonov AV, Sayan BS, Tucci P, Agostini M, Mineo TC, Levine AJ, Melino G (2014) p73 regulates serine biosynthesis in cancer. Oncogene 33 (42):5039-5046. doi:10.1038/onc.2013.456

51. Sen N, Cross AM, Lorenzi PL, Khan J, Gryder BE, Kim S, Caplen NJ (2018) EWS-FLI1 reprograms the metabolism of Ewing sarcoma cells via positive regulation of glutamine import and serine-glycine biosynthesis. Mol Carcinog 57 (10):1342-1357. doi:10.1002/mc.22849

52. Sun L, Song L, Wan Q, Wu G, Li X, Wang Y, Wang J, Liu Z, Zhong X, He X, Shen S, Pan X, Li A, Wang Y, Gao P, Tang H, Zhang H (2015) cMyc-mediated activation of serine biosynthesis pathway is critical for cancer progression under nutrient deprivation conditions. Cell Res 25 (4):429-444. doi:10.1038/cr.2015.33 53. Kremer JC, Prudner BC, Lange SES, Bean GR, Schultze MB, Brashears CB, Radyk MD, Redlich N, Tzeng SC, Kami K, Shelton L, Li A, Morgan Z, Bomalaski JS, Tsukamoto T, McConathy J, Michel LS, Held JM, Van Tine BA (2017) Arginine Deprivation Inhibits the Warburg Effect and Upregulates Glutamine Anaplerosis and Serine Biosynthesis in ASS1-Deficient Cancers. Cell Rep 18 (4):991-1004. doi:10.1016/j.celrep.2016.12.077

54. Ye J, Mancuso A, Tong X, Ward PS, Fan J, Rabinowitz JD, Thompson CB (2012) Pyruvate kinase M2 promotes de novo serine synthesis to sustain mTORC1 activity and cell proliferation. Proc Natl Acad Sci U S A 109 (18):6904-6909. doi:10.1073/pnas.1204176109

55. Samanta D, Park Y, Andrabi SA, Shelton LM, Gilkes DM, Semenza GL (2016) PHGDH Expression Is Required for Mitochondrial Redox Homeostasis, Breast Cancer Stem Cell Maintenance, and Lung Metastasis. Cancer Res 76 (15):44304442. doi:10.1158/0008-5472.CAN-16-0530 
56. Fang Y, Liang X, Xu J, Cai X (2018) miR-424 targets AKT3 and PSAT1 and has a tumor-suppressive role in human colorectal cancer. Cancer Manag Res 10:6537-6547. doi:10.2147/CMAR.S185789

57. Sun C ZX, Chen Y, Jia Q, Yang J, Shu Y (2018) MicroRNA-365 suppresses cell growth and invasion in esophageal squamous cell carcinoma by modulating phosphoserine aminotransferase 1. Cancer Manag Res 10:4581-4590. doi:10.2147/CMAR.S157858

58. Yan S JH, Fang S, Yin F, Wang Z, Jia Y, Sun X, Wu S, Jiang T, Mao A. (2015) MicroRNA-340 Inhibits Esophageal Cancer Cell Growth and Invasion by Targeting Phosphoserine Aminotransferase 1. Cell Physiol Biochem 37 (1):375-386. doi:10.1159/000430361

59. Wang X, Min S, Liu H, Wu N, Liu X, Wang T, Li W, Shen Y, Wang H, Qian Z, $\mathrm{Xu} \mathrm{H}$, Zhao C, Chen Y (2019) Nf1 loss promotes Kras-driven lung adenocarcinoma and results in Psat1-mediated glutamate dependence. EMBO Mol Med 11 (6). doi:10.15252/emmm.201809856

60. Yang CS, Stampouloglou E, Kingston NM, Zhang L, Monti S, Varelas X (2018) Glutamine-utilizing transaminases are a metabolic vulnerability of TAZ/YAPactivated cancer cells. EMBO Rep 19 (6). doi:10.15252/embr.201643577

61. Cordenonsi M, Zanconato F, Azzolin L, Forcato M, Rosato A, Frasson C, Inui M, Montagner M, Parenti AR, Poletti A, Daidone MG, Dupont S, Basso G, Bicciato S, Piccolo S (2011) The Hippo transducer TAZ confers cancer stem cell-related traits on breast cancer cells. Cell 147 (4):759-772. doi:10.1016/j.cell.2011.09.048 62. Coloff JL, Murphy JP, Braun CR, Harris IS, Shelton LM, Kami K, Gygi SP, Selfors LM, Brugge JS (2016) Differential Glutamate Metabolism in Proliferating and Quiescent Mammary Epithelial Cells. Cell Metab 23 (5):867-880. doi:10.1016/j.cmet.2016.03.016

63. Kottakis F, Nicolay BN, Roumane A, Karnik R, Gu H, Nagle JM, Boukhali M, Hayward MC, Li YY, Chen T, Liesa M, Hammerman PS, Wong KK, Hayes DN, Shirihai OS, Dyson NJ, Haas W, Meissner A, Bardeesy N (2016) LKB1 loss links serine metabolism to DNA methylation and tumorigenesis. Nature 539 (7629):390395. doi:10.1038/nature20132

64. Sun WY, Kim HM, Jung WH, Koo JS (2016) Expression of serine/glycine metabolism-related proteins is different according to the thyroid cancer subtype. $J$ Transl Med 14 (1):168. doi:10.1186/s12967-016-0915-8

65. Liao KM, Chao TB, Tian YF, Lin CY, Lee SW, Chuang HY, Chan TC, Chen TJ, Hsing CH, Sheu MJ, Li CF (2016) Overexpression of the PSAT1 Gene in Nasopharyngeal Carcinoma Is an Indicator of Poor Prognosis. J Cancer 7 (9):1088-1094. doi:10.7150/jca.15258

66. Ravez S, Spillier Q, Marteau R, Feron O, Frederick R (2017) Challenges and Opportunities in the Development of Serine Synthetic Pathway Inhibitors for $\begin{array}{lllll}\text { Cancer Therapy. J Med } & \text { Chem } 60 & \text { (4):1227-1237. }\end{array}$ doi:10.1021/acs.jmedchem.6b01167

67. Locasale JW, Grassian AR, Melman T, Lyssiotis CA, Mattaini KR, Bass AJ, Heffron G, Metallo CM, Muranen T, Sharfi H, Sasaki AT, Anastasiou D, Mullarky E, Vokes NI, Sasaki M, Beroukhim R, Stephanopoulos G, Ligon AH, Meyerson M, Richardson AL, Chin L, Wagner G, Asara JM, Brugge JS, Cantley LC, Vander 
Heiden MG (2011) Phosphoglycerate dehydrogenase diverts glycolytic flux and contributes to oncogenesis. Nat Genet 43 (9):869-874. doi:10.1038/ng.890

68. Ross KC, Andrews AJ, Marion CD, Yen TJ, Bhattacharjee V (2017) Identification of the Serine Biosynthesis Pathway as a Critical Component of BRAF Inhibitor Resistance of Melanoma, Pancreatic, and Non-Small Cell Lung Cancer Cells. Mol Cancer Ther 16 (8):1596-1609. doi:10.1158/1535-7163.MCT-16-0798

69. Zaal EA, Wu W, Jansen G, Zweegman S, Cloos J, Berkers CR (2017) Bortezomib resistance in multiple myeloma is associated with increased serine synthesis. Cancer Metab 5:7. doi:10.1186/s40170-017-0169-9

70. Nwosu ZC, Battello N, Rothley M, Pioronska W, Sitek B, Ebert MP, Hofmann U, Sleeman J, Wolfl S, Meyer C, Megger DA, Dooley S (2018) Liver cancer cell lines distinctly mimic the metabolic gene expression pattern of the corresponding human tumours. J Exp Clin Cancer Res 37 (1):211. doi:10.1186/s13046-0180872-6

71. Jiang J, Zhang L, Chen $\mathrm{H}$, Lei $Y$, Zhang $T$, Wang $Y$, Jin $P$, Lan J, Zhou L, Huang Z, Li B, Liu Y, Gao W, Xie K, Zhou L, Nice EC, Peng Y, Cao Y, Wei Y, Wang K, Huang C (2019) Regorafenib induces lethal autophagy arrest by stabilizing PSAT1 in glioblastoma. Autophagy:1-17. doi:10.1080/15548627.2019.1598752

72. Liu B, Jia Y, Cao Y, Wu S, Jiang H, Sun X, Ma J, Yin X, Mao A, Shang M (2016) Overexpression of Phosphoserine Aminotransferase 1 (PSAT1) Predicts Poor Prognosis and Associates with Tumor Progression in Human Esophageal Squamous Cell Carcinoma. Cell Physiol Biochem 39 (1):395-406. doi:10.1159/000445633

73. Vie N, Copois V, Bascoul-Mollevi C, Denis V, Bec N, Robert B, Fraslon C, Conseiller E, Molina F, Larroque C, Martineau P, Del Rio M, Gongora C (2008) Overexpression of phosphoserine aminotransferase PSAT1 stimulates cell growth and increases chemoresistance of colon cancer cells. Mol Cancer 7:14. doi:10.1186/1476-4598-7-14

74. Yoon S, Kim JG, Seo AN, Park SY, Kim HJ, Park JS, Choi GS, Jeong JY, Jun do Y, Yoon GS, Kang BW (2015) Clinical Implication of Serine MetabolismAssociated Enzymes in Colon Cancer. Oncology 89 (6):351-359. doi:10.1159/000439571

75. Qian C, Xia Y, Ren Y, Yin Y, Deng A (2017) Identification and validation of PSAT1 as a potential prognostic factor for predicting clinical outcomes in patients with colorectal carcinoma. Oncol Lett 14 (6):8014-8020. doi:10.3892/ol.2017.7211 76. Maddocks ODK, Athineos D, Cheung EC, Lee P, Zhang T, van den Broek NJF, Mackay GM, Labuschagne CF, Gay D, Kruiswijk F, Blagih J, Vincent DF, Campbell KJ, Ceteci F, Sansom OJ, Blyth K, Vousden KH (2017) Modulating the therapeutic response of tumours to dietary serine and glycine starvation. Nature 544 (7650):372-376. doi:10.1038/nature22056

77. Dai J, Wei R, Zhang P, Kong B (2019) Overexpression of microRNA-195-5p reduces cisplatin resistance and angiogenesis in ovarian cancer by inhibiting the PSAT1-dependent GSK3beta/beta-catenin signaling pathway. J TransI Med 17 (1):190. doi:10.1186/s12967-019-1932-1 
78. Toyama A, Suzuki A, Shimada T, Aoki C, Aoki Y, Umino Y, Nakamura Y, Aoki D, Sato TA (2012) Proteomic characterization of ovarian cancers identifying annexin-A4, phosphoserine aminotransferase, cellular retinoic acid-binding protein 2, and serpin B5 as histology-specific biomarkers. Cancer Sci 103 (4):747-755. doi:10.1111/j.1349-7006.2012.02224.x

79. Zheng MJ LX, Hu YX, Dong H, Gou R, Nie X, Liu Q, Ying-Ying H, Liu JJ, Lin B (2019) Identification of molecular marker associated with ovarian cancer prognosis using bioinformatics analysis and experiments. J Cell Physiol 234 (7):1102311036. doi: $10.1002 / j c p .2792$

80. Zhang $B$, Zheng A, Hydbring $P$, Ambroise G, Ouchida AT, Goiny M, Vakifahmetoglu-Norberg H, Norberg E (2017) PHGDH Defines a Metabolic Subtype in Lung Adenocarcinomas with Poor Prognosis. Cell Rep 19 (11):22892303. doi:10.1016/j.celrep.2017.05.067

81. Yang Y, Wu J, Cai J, He Z, Yuan J, Zhu X, Li Y, Li M, Guan H (2015) PSAT1 regulates cyclin $\mathrm{D} 1$ degradation and sustains proliferation of non-small cell lung cancer cells. Int J Cancer 136 (4):E39-50. doi:10.1002/ijc.29150

82. Martens JWMN, I.; Koenig, T.; Look, M.P.; Harbeck, N.; Model, F.; Kluth, A.; Vries, J.B.; Sieuwerts, A.M.; Portengen,H.; Meijer-Van Gelder, M.E.; Piepenbrock, C.; Olek, A.; Hofler, H.; Kiechle, M.; Klijn, J.G.M.; Schnitt, M.; Maier, S.; Foekens, J.A. (2005) Association of DNA Methylation of Phosphoserine Aminotransferase with Response to Endocrine Therapy in Patients with Recurrent Breast Cancer. Cancer Res 65 (10):4101-4107

83. Antonov AA, M.; Morello, M.; Minieri, M.; Melino, G.; Amelio, I. (2014) Bioinformatics analysis of the serine and glycine pathway in cancer cells. Oncotarget 5 (22):11004-11013

84. Pollari SK, S.; Edgren, H.; Wolf, M.; Kohonen, P.; Sara, H.; Guise, T.; Nees, M.; Kallioniemi, O. (2011) Enhanced serine production by bone metastatic breast cancer cells stimulates osteoclastogenesis. Breast Cancer Res Treat (125):421430. doi:10.1007/s10549-010-0848-5

85. Park SB, Chung CK, Gonzalez E, Yoo C (2018) Causal Inference Network of Genes Related with Bone Metastasis of Breast Cancer and Osteoblasts Using Causal Bayesian Networks. J Bone Metab 25 (4):251-266. doi:10.11005/jbm.2018.25.4.251

86. Pacold ME, Brimacombe KR, Chan SH, Rohde JM, Lewis CA, Swier LJ, Possemato R, Chen WW, Sullivan LB, Fiske BP, Cho S, Freinkman E, Birsoy K, Abu-Remaileh M, Shaul YD, Liu CM, Zhou M, Koh MJ, Chung H, Davidson SM, Luengo A, Wang AQ, Xu X, Yasgar A, Liu L, Rai G, Westover KD, Vander Heiden MG, Shen M, Gray NS, Boxer MB, Sabatini DM (2016) A PHGDH inhibitor reveals coordination of serine synthesis and one-carbon unit fate. Nat Chem Biol 12 (6):452-458. doi:10.1038/nchembio.2070

87. Possemato R, Marks KM, Shaul YD, Pacold ME, Kim D, Birsoy K, Sethumadhavan S, Woo HK, Jang HG, Jha AK, Chen WW, Barrett FG, Stransky N, Tsun ZY, Cowley GS, Barretina J, Kalaany NY, Hsu PP, Ottina K, Chan AM, Yuan B, Garraway LA, Root DE, Mino-Kenudson M, Brachtel EF, Driggers EM, Sabatini DM (2011) Functional genomics reveal that the serine synthesis pathway 
is essential in breast cancer. Nature 476 (7360):346-350. doi:10.1038/nature10350

88. Geck RC, Toker A (2016) Nonessential amino acid metabolism in breast cancer. Adv Biol Regul 62:11-17. doi:10.1016/j.jbior.2016.01.001

89. Chen J, Chung, F., Yang, G., Pu, M., Gao, H., Jiang, W., Yin, H., Capka, V., Kasibhatla, S., Laffittee, B., Jaeger, S., Pagliarini, R., Chen, Y., Zhou, W. (2013) Phosphoglycerate dehydrogenase is dispensable for breast cancer maintenance and growth. Oncotarget 4 (12):2502-2511

90. Semenza GL (2016) Hypoxia-inducible factors: coupling glucose metabolism and redox regulation with induction of the breast cancer stem cell phenotype. The EMBO Journal 36 (3):252-259. doi:10.15252/embj.201695204

91. Samanta D, Semenza GL (2016) Serine Synthesis Helps Hypoxic Cancer Stem Cells Regulate Redox. Cancer Res 76 (22):6458-6462. doi:10.1158/00085472.CAN-16-1730

92. Kwon JE, Kim DH, Jung WH, Koo JS (2014) Expression of serine and glycinerelated enzymes in phyllodes tumor. Neoplasma 61 (5):566-578. doi:10.4149/neo 2014069

93. Kim YH, Jung, W.H., Koo, J.S. (2014) Expression of metabolism-related proteins in invasive lobular carcinoma: comparison to invasive ductal carcinoma. Tumor Biology 35:10381-10393. doi:10.1007/s13277-014-2345-7

94. Fackler MJ, Umbricht CB, Williams D, Argani P, Cruz LA, Merino VF, Teo WW, Zhang Z, Huang P, Visvananthan K, Marks J, Ethier S, Gray JW, Wolff AC, Cope LM, Sukumar S (2011) Genome-wide methylation analysis identifies genes specific to breast cancer hormone receptor status and risk of recurrence. Cancer Res 71 (19):6195-6207. doi:10.1158/0008-5472.CAN-11-1630

95. Bu D, Lewis CM, Sarode V, Chen M, Ma X, Lazorwitz AM, Rao R, Leitch M, Moldrem A, Andrews V, Gazdar A, Euhus D (2013) Identification of breast cancer DNA methylation markers optimized for fine-needle aspiration samples. Cancer Epidemiol Biomarkers Prev 22 (12):2212-2221. doi:10.1158/1055-9965.EPI-130208

96. Salta S, S PN, Fontes-Sousa M, Lopes P, Freitas M, Caldas M, Antunes L, Castro F, Antunes P, Palma de Sousa S, Henrique R, Jeronimo C (2018) A DNA Methylation-Based Test for Breast Cancer Detection in Circulating Cell-Free DNA. J Clin Med 7 (11). doi:10.3390/jcm7110420

97. De Marchi T, Timmermans MA, Sieuwerts AM, Smid M, Look MP, Grebenchtchikov N, Sweep F, Smits JG, Magdolen V, van Deurzen CHM, Foekens JA, Umar A, Martens JW (2017) Phosphoserine aminotransferase 1 is associated to poor outcome on tamoxifen therapy in recurrent breast cancer. Sci Rep 7 (1):2099. doi:10.1038/s41598-017-02296-w

98. Singh R, Dagar P, Pal S, Basu B, Shankar BS (2018) Significant alterations of the novel 15 gene signature identified from macrophage-tumor interactions in breast cancer. Biochim Biophys Acta Gen Subj 1862 (3):669-683. doi:10.1016/j.bbagen.2017.12.004

99. Yang S, Zhang JJ, Huang XY (2012) Mouse models for tumor metastasis. Methods Mol Biol 928:221-228. doi:10.1007/978-1-62703-008-3_17 
100. Hiemer SE, Szymaniak AD, Varelas $X$ (2014) The transcriptional regulators TAZ and YAP direct transforming growth factor beta-induced tumorigenic phenotypes in breast cancer cells. J Biol Chem 289 (19):13461-13474. doi:10.1074/jbc.M113.529115

101. Zanconato F, Cordenonsi M, Piccolo S (2016) YAP/TAZ at the Roots of Cancer. Cancer Cell 29 (6):783-803. doi:10.1016/j.ccell.2016.05.005

102. Qiao M, Sheng S, Pardee AB (2008) Metastasis and AKT activation. Cell Cycle 7 (19):2991-2996. doi:10.4161/cc.7.19.6784

103. Nakopoulou L, Katsarou S, Giannopoulou I, Alexandrou P, Tsirmpa I, Panayotopoulou E, Mavrommatis J, Keramopoulos A (2002) Correlation of tissue inhibitor of metalloproteinase-2 with proliferative activity and patients' survival in breast cancer. Mod Pathol 15 (1):26-34. doi:10.1038/modpathol.3880486

104. Tetu B, Brisson J, Wang CS, Lapointe H, Beaudry G, Blanchette C, Trudel D (2006) The influence of MMP-14, TIMP-2 and MMP-2 expression on breast cancer prognosis. Breast Cancer Res 8 (3):R28. doi:10.1186/bcr1503

105. Melani M, Simpson KJ, Brugge JS, Montell D (2008) Regulation of cell adhesion and collective cell migration by hindsight and its human homolog RREB1. Curr Biol 18 (7):532-537. doi:10.1016/j.cub.2008.03.024

106. Riggs BL, Hartmann LC (2003) Selective estrogen-receptor modulators -mechanisms of action and application to clinical practice. N Engl J Med 348 (7):618-629. doi:10.1056/NEJMra022219

107. Osborne CK, Wakeling A, Nicholson RI (2004) Fulvestrant: an oestrogen receptor antagonist with a novel mechanism of action. Br J Cancer 90 Suppl 1:S26. doi:10.1038/sj.bjc.6601629

108. Bronzert DAG, Geoffrey L.; Lippman, Marc E. (1985) Selection and characterization of a breast cancer cell line resistant to the antiestrogen LY 117018. Endocrinology 117 (4):1409-1417

109. Brunner NB, Birgitte; Jirus, Stana; Skaar, Todd C.; Holst-Hansen, Claus; Lippman, Jeremy; Frandsen, Thomas; Spang-Thomsen, Mogens; Fugua, Suzanne A. W.; Clarke, Robert (1997) MCF7/LCC9: An antiestrogen-resistant MCF-7 variant in which acquired resistance to the sterodial antiestrogen $\mathrm{ICl}$ 182,780 confers an early cross-resitance to the nonsteroidal antiestrogen tamoxifen. Cancer Research 57:3486-3493

110. Astruc ME, Chabret C, Bali P, Gagne D, Pons M (1995) Prolonged treatment of breast cancer cells with antiestrogens increases the activating protein-1mediated response: involvement of the estrogen receptor. Endocrinology 136 (3):824-832. doi:10.1210/endo.136.3.7867590

111. Wittliff JL, Kruer TL, Andres SA, Smolenkova I (2008) Molecular signatures of estrogen receptor-associated genes in breast cancer predict clinical outcome. Adv Exp Med Biol 617:349-357. doi:10.1007/978-0-387-69080-3_33

112. Mihaly Z, Kormos M, Lanczky A, Dank M, Budczies J, Szasz MA, Gyorffy B (2013) A meta-analysis of gene expression-based biomarkers predicting outcome after tamoxifen treatment in breast cancer. Breast Cancer Res Treat 140 (2):219232. doi:10.1007/s10549-013-2622-y

113. Gyorffy B, Lanczky A, Eklund AC, Denkert C, Budczies J, Li Q, Szallasi Z (2010) An online survival analysis tool to rapidly assess the effect of 22,277 genes 
on breast cancer prognosis using microarray data of 1,809 patients. Breast Cancer Res Treat 123 (3):725-731. doi:10.1007/s10549-009-0674-9

114. Brunner N, Boysen B, Jirus S, Skaar TC, Holst-Hansen C, Lippman J, Frandsen T, Spang-Thomsen M, Fuqua SA, Clarke R (1997) MCF7/LCC9: an antiestrogen-resistant MCF-7 variant in which acquired resistance to the steroidal antiestrogen ICl 182,780 confers an early cross-resistance to the nonsteroidal antiestrogen tamoxifen. Cancer Res 57 (16):3486-3493

115. Chen Z, Wang Y, Warden C, Chen S (2015) Cross-talk between ER and HER2 regulates C-MYC-mediated glutamine metabolism in aromatase inhibitor resistant breast cancer cells. J Steroid Biochem Mol Biol 149:118-127. doi:10.1016/j.jsbmb.2015.02.004

116. McNeil CM, Sergio CM, Anderson LR, Inman CK, Eggleton SA, Murphy NC, Millar EK, Crea P, Kench JG, Alles MC, Gardiner-Garden M, Ormandy CJ, Butt AJ, Henshall SM, Musgrove EA, Sutherland RL (2006) c-Myc overexpression and endocrine resistance in breast cancer. J Steroid Biochem Mol Biol 102 (1-5):147155. doi:10.1016/j.jsbmb.2006.09.028

117. Newman AC, Maddocks ODK (2017) Serine and Functional Metabolites in Cancer. Trends Cell Biol 27 (9):645-657. doi:10.1016/j.tcb.2017.05.001

118. Mullarky E, Lucki NC, Beheshti Zavareh R, Anglin JL, Gomes AP, Nicolay BN, Wong JC, Christen S, Takahashi H, Singh PK, Blenis J, Warren JD, Fendt SM, Asara JM, DeNicola GM, Lyssiotis CA, Lairson LL, Cantley LC (2016) Identification of a small molecule inhibitor of 3-phosphoglycerate dehydrogenase to target serine biosynthesis in cancers. Proc Natl Acad Sci U S A 113 (7):17781783. doi:10.1073/pnas. 1521548113 


\author{
CURRICULUM VITAE \\ Stephanie Metcalf \\ 11518 Yorktown Blvd \\ Sellersburg, IN 47172 \\ 812-725-3709 \\ s.a.metcalf22@gmail.com
}

\title{
EDUCATION
}

May 2014 B.A. in Chemistry, Indiana University-Purdue University Indianapolis, IN

August 2017 M.S. Biochemistry and Molecular Genetics, University of Louisville, Louisville, KY

Dec. 2019 Ph.D. University of Louisville, Louisville, KY

\section{RESEARCH POSITIONS}

Indiana University-Purdue University Indianapolis

- Research Intern - Dr. Marc Mendonca's Laboratory, Indiana University School of Medicine (August 2012 - May 2013)

- Undergraduate Student Researcher - IUPUI Center for Teaching and Learning (May 2013 - August 2013)

- Undergraduate Student Researcher - Dr. Marc Mendonca's Laboratory, Indiana University School of Medicine (August 2013 - May 2014)

University of Louisville

- Graduate Research Assistant - Dr. Brian Clem's Laboratory (March 2015 - Present)

\section{ABSTRACTS AND PRESENTATIONS}

Select Poster Presentations

- The Role of PSAT1 in Triple Negative Breast Cancer Metastasis; Stephanie Metcalf, Traci Kruer, Susan Dougherty, Rumeysa Biyik-Sit, Carolyn Klinge, Brian Clem

- AACR; Atlanta, GA 2019

○ Research!Louisville; Louisville, KY 2018

- Investigation of Phosphoserine Aminotransferase 1 and its Role in Breast Cancer Progression; Stephanie Metcalf, Traci Kruer, Carolyn Klinge, James Wittliff, Brian Clem

- San Antonio Breast Cancer Symposium; San Antonio, TX; 2017

- Research!Louisville; Louisville, KY; 2017

○ BMG Departmental Retreat; Louisville, KY; 2017

- Phosphoserine Aminotransferase (PSAT1) Promotes Metastatic Characteristics in Human Breast Cancer; Stephanie Metcalf, Traci Kruer, Carolyn Klinge, Brian Clem

○ Research!Louisville; Louisville, KY; 2016

- Putative Role of Phosphoserine Aminotransferase (PSAT1) in the Metastatic Potential of Human Breast Cancer; Stephanie Metcalf, Traci Kruer, Carolyn Klinge, Brian Clem

○ Research!Louisville; Louisville, KY; 2015

○ Metastatic Breast Cancer Symposium; Houston, TX; 2015 


\section{O}

○ BMG Departmental Retreat; Louisville, KY; 2015

\section{Oral Presentations}

- Investigation of Phosphoserine Aminotransferase 1 and its Role in Breast Cancer Progression; Stephanie Metcalf, Traci Kruer, Carolyn Klinge, James Wittliff, Brian Clem; BMG Departmental Retreat; Louisville, KY; 2017

\section{PUBLICATIONS}

Published

- McDaniel, J., Metcalf, S., Sours, J., Janke, T., Newbrough, J., Shuck, L., \& Varma-Nelson, P. (2013). Supporting student collaboration in cyberspace: A cpltl study of web conferencing platforms. Educause Review Online, (September/October), Retrieved from http://www.educause.edu/ero/article/supporting-student-collaboration-cyberspaceSubmitted cpltt-study-web-conferencing-platforms

- Metcalf, S., Dougherty, S., Kruer, T., Clem, B. F. Selective loss of Phosphoserine Aminotransferase (PSAT1) suppresses triple negative breast cancer metastasis. Experimental and Clinical Metastasis.

\section{TEACHING ACTIVITIES}

University of Louisville, School of Medicine, Louisville, KY

- Teaching Assistant - Graduate Student Seminar Class, 2017

- Teaching Assistant - Advanced Biochemistry II, 2016 and 2018

- Student Mentor for summer undergraduate and high school students 2015, 2016 and 2019 Indiana University-Purdue University Indianapolis, Indianapolis, IN

- Peer Leader - General Chemistry $1^{\text {st }}$ Semester, 2011 - 2014

- Online Peer Leader - General Chemistry $1^{\text {st }}$ Semester, 2013 - 2014

\section{LEADERSHIP EXPERIENCE}

- School of Science Ambassador Leadership Team, IUPUI, Indianapolis, IN; Events Committee Co-Chair, Executive Committee Co-Chair, President (November 2011 - June 2014)

- Undergraduate Women in Science, IUPUI Women in Science Program, Indianapolis, IN; Academic and Pre-Professional Coordinator (August 2013 - May 2014)

- Graduate Student Council, UofL, Louisville, KY; Departmental Representative (August 2016 - May 2017)

\section{HONORS AND AWARDS}

August 2010 - May 2014

August 2010 - May 2014

Nov. 2011 - Dec. 2013

IUPUI

September 2015

Award

April 2019
Chancellor Scholar, IUPUI Honors College, IUPUI Academic Excellence Scholarship Recipient, IUPUI Science Ambassador Leadership Team Scholarship Recipient, Biochemistry and Molecular Genetics Departmental Travel UofL Graduate Student Council Travel Award 


\section{COMMUNITY SERVICE}

- Town of Borden, Borden, IN (August 2007 - December 2016)

- Borden Lions Club, Borden, IN (August 2007 - December 2017)

- Indiana State Museum, Indianapolis, IN (December 2011 - May 2014)

- Bio-Rad Ambassador, Louisville, KY (January 2018 - present)

- William W. Borden Jr/Sr High School, Borden, IN (January 2019 - present)

\section{MEMBERSHIPS}

- American Association of Cancer Research

- American Chemical Society

- Alpha Lambda Delta Honor Society

- Phi Eta Sigma Honor Society 